Estudo, avaliação e comparação de técnicas de detecção não supervisionada de outliers 



\title{
Estudo, avaliação e comparação de técnicas de detecção não supervisionada de outliers ${ }^{1}$
}

\section{Guilherme Oliveira Campos}

Orientador: Prof. Dr. Ricardo José Gabrielli Barreto Campello

\begin{abstract}
Dissertação apresentada ao Instituto de Ciências Matemáticas e de Computação - ICMC-USP, como parte dos requisitos para obtenção do título de Mestre em Ciências - Ciências de Computação e Matemática Computacional. VERSÃO REVISADA
\end{abstract}

USP - São Carlos

Abril de 2015

\footnotetext{
${ }^{1}$ Trabalho realizado com o auxílio financeiro da CNPq e CAPES
} 
Ficha catalográfica elaborada pela Biblioteca Prof. Achille Bassi e Seção Técnica de Informática, ICMC/USP, com os dados fornecidos pelo(a) autor(a)

\section{Oliveira Campos, Guilherme}

0198e Estudo, avaliação e comparação de técnicas de deteç̧ão não supervisionada de outliers / Guilherme Oliveira Campos; orientador Ricardo José Gabrielli Barreto Campello. -- São Carlos, 2015. $67 \mathrm{p}$.

Dissertação (Mestrado - Programa de Pós-Graduação em Ciências de Computação e Matemática Computacional) -- Instituto de Ciências Matemáticas e de Computação, Universidade de São Paulo, 2015.

1. Mineração de Dados. 2. Deteç̧ão de Outliers. 3. Benchmark de Bases de Dados. 4. Avaliação de Algoritmos. 5. Seleção de Atributos. I. José Gabrielli Barreto Campello, Ricardo, orient. II. Título. 
Aos meus pais. 



\section{Agradecimentos}

À minha família, em especial ao meu pai Gilson Campos de Carvalho, minha mãe Márcia Cândida de Oliveira Campos e meus irmãos Gustavo Oliveira Campos e Gabriel Oliveira Campos pelo incentivo, amor e confiança cruciais para meu desenvolvimento profissional e pessoal.

À todos os meus amigos que me acompanharam nesta jornada árdua pelas trocas de experiência, apoio e amizade.

Ao meu orientador Prof. Dr. Ricardo José Gabrielli Barreto Campello pela orientação impecável.

Aos professores Arthur Zimek e Joerg Sander pela contribuição intelectual importantíssima para desenvolver um trabalho de grande qualidade científica.

Ao CNPq e CAPES pelo suporte financeiro para a realização deste trabalho.

À todos que, de forma direta ou indireta, contribuíram para a realização deste trabalho. 



\section{Resumo}

A área de detecção de outliers (ou detecção de anomalias) possui um papel fundamental na descoberta de padrões em dados que podem ser considerados excepcionais sob alguma perspectiva. Detectar tais padrões é relevante de maneira geral porque, em muitas aplicações de mineração de dados, tais padrões representam comportamentos extraordinários que merecem uma atenção especial. Uma importante distinção se dá entre as técnicas supervisionadas e não supervisionadas de detecção. O presente projeto enfoca as técnicas de detecção não supervisionadas. Existem dezenas de algoritmos desta categoria na literatura e novos algoritmos são propostos de tempos em tempos, porém cada um deles utiliza uma abordagem própria do que deve ser considerado um outlier ou não, que é um conceito subjetivo no contexto não supervisionado. Isso dificulta sensivelmente a escolha de um algoritmo em particular em uma dada aplicação prática. Embora seja de conhecimento comum que nenhum algoritmo de aprendizado de máquina pode ser superior a todos os demais em todos os cenários de aplicação, é uma questão relevante se o desempenho de certos algoritmos em geral tende a dominar o de determinados outros, ao menos em classes particulares de problemas. Neste projeto, propõe-se contribuir com o estudo, seleção e pré-processamento de bases de dados que sejam apropriadas para se juntarem a uma coleção de benchmarks para avaliação de algoritmos de detecção não supervisionada de outliers. Propõe-se ainda avaliar comparativamente o desempenho de métodos de detecção de outliers. Durante parte do meu trabalho de mestrado, tive a colaboração intelectual de Erich Schubert, Ira Assent, Barbora Micenková, Michael Houle e, principalmente, Joerg Sander e Arthur Zimek. A contribuição deles foi essencial para as análises dos resultados e a forma compacta de apresentá-los. 



\section{Abstract}

The outlier detection area has an essential role in discovering patterns in data that can be considered as exceptional in some perspective. Detect such patterns is important in general because, in many data mining applications, such patterns represent extraordinary behaviors that deserve special attention. An important distinction occurs between supervised and unsupervised detection techniques. This project focuses on the unsupervised detection techniques. There are dozens of algorithms in this category in literature and new algorithms are proposed from time to time, but each of them uses its own approach of what should be considered an outlier or not, which is a subjective concept in the unsupervised context. This considerably complicates the choice of a particular algorithm in a given practical application. While it is common knowledge that no machine learning algorithm can be superior to all others in all application scenarios, it is a relevant question if the performance of certain algorithms in general tends to dominate certain other, at least in particular classes of problems. In this project, proposes to contribute to the databases study, selection and pre-processing that are appropriate to join a benchmark collection for evaluating unsupervised outlier detection algorithms. It is also proposed to evaluate comparatively the performance of outlier detection methods. During part of my master thesis, I had the intellectual collaboration of Erich Schubert, Ira Assent, Barbora Micenková, Michael Houle and especially Joerg Sander and Arthur Zimek. Their contribution was essential for the analysis of the results and the compact way to present them. 



\section{Conteúdo}

Lista de Figuras $\quad$ xii

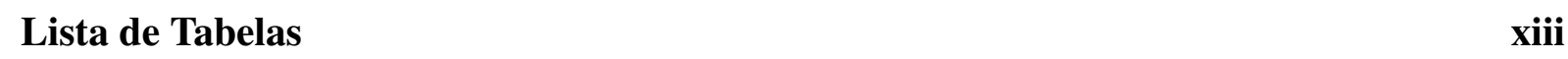

$\begin{array}{ll}\text { Lista de Abreviaturas } & \text { xv }\end{array}$

\begin{tabular}{lll}
\hline 1 & Introdução & 1
\end{tabular}

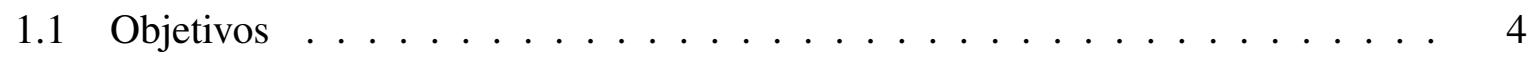

1.2 Organização do Trabalho $\ldots \ldots \ldots \ldots \ldots \ldots$

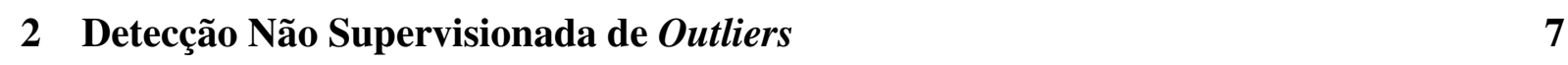

$2.1 \quad$ Algoritmos de Detecção de Outliers Globais . . . . . . . . . . . . . . . . . 8

$2.1 .1 \quad k$-Nearest Neighbors $(\mathrm{KNND}] \ldots \ldots \ldots$. . . . . . . . . 8

$2.1 .2 \quad k$-Nearest Neighbors Weighted (KNNW] . . . . . . . . . . . . . . 8

$2.1 .3 \quad$ Outlier Detection using Indegree Number (ODIN) . . . . . . . . . . 9

2.2 Algoritmos de Detecção de Outliers Locais . . . . . . . . . . . . . . . . . . 10

$2.2 .1 \quad$ Local Outlier Factor $(\mathrm{LOF})$. . . . . . . . . . . . . . . . . . . . . 10

2.2.2 Simplified Local Outlier Factor (Simplified LOF] . . . . . . . . . . . . 12

2.2 .3 Local Distance-based Outlier Factor $(\underline{\mathrm{LDOF}})$. . . . . . . . . . . . . . 12

2.2 .4 Local Outlier Probabilities $(\mathrm{LoOP})$. . . . . . . . . . . . . . . 13

2.2 .5 Influenced Outlierness (INFLO) . . . . . . . . . . . . . . . . 15

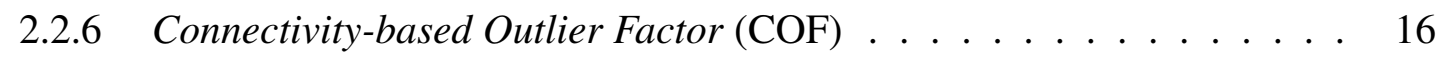

2.2 .7 Local Density Factor (LDF) . . . . . . . . . . . . . . . . . . . . . . . . 16

$2.2 .8 \quad$ Kernel Density Estimates Outlier Score (KDEOS) . . . . . . . . . . . 17

$2.2 .9 \quad$ Fast Angle-based Outlier Detection (Fast ABOD) . . . . . . . . . . . . 17

2.3 Avaliação de Algoritmos de Detecção de Outliers . . . . . . . . . . . . . . . 18

3 Benchmarks de Bases de Dados para Detecção de Outliers 23

3.1 Definição e Motivação . . . . . . . . . . . . . . . . . . . . . 23

3.2 Propostas na Literatura $\ldots \ldots \ldots \ldots$. . . . . . . . . . . . . . . 24 


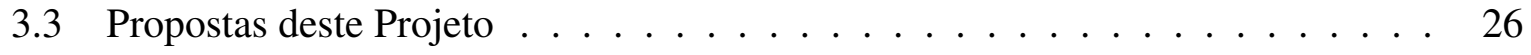

3.3.1 Análise e Pré-Seleção de Bases de Dados . . . . . . . . . . . . . . . . . . . . . . . . . . 27

3.3 .2 Pré-Processamento . . . . . . . . . . . . . . . . . 27

3.3.3 Aplicação de Algoritmos Representativos de Detecção de Outliers nas Bases e Geração de Estatísticas de Interesse . . . . . . . . . . . . . 29

3.3 .4 Seleção de Atributos . . . . . . . . . . . . . . . . . 30

$\begin{array}{lll}4 & \text { Resultados } & 31\end{array}$

4.1 Bases de Dados . . . . . . . . . . . . . . . . . . . 31

$4.1 .1 \quad$ Bases de Dados com Semântica Clara entre Outliers e Inliers. . . . . . . 31

4.1.2 Bases de Dados Utilizadas na Literatura Independente da Semântica . . 35

4.2 Execução de Algoritmos de Detecção de Outliers . . . . . . . . . . . . . . . . 40

4.2.1 Caracterização dos Métodos de Acordo com os Resultados . . . . . . . 41

$4.2 .2 \quad$ Caracterização das Bases de Dados de Acordo com os Resultados . . . 45

4.2 .3 Pós-processamento . . . . . . . . . . . . . . . . . 52

$\begin{array}{lll}5 \text { Conclusão } & 57\end{array}$

\begin{tabular}{|lll}
\hline A & Resultados AUC & 59
\end{tabular}

\begin{tabular}{|l|l}
\hline Referências Bibliográficas & 63
\end{tabular} 


\section{Lista de Figuras}

2.1 Dois pontos pertencentes a regiões de densidades distintas, porém com o mesmo valor de $D^{k}(k=8)($ Inspirado em $($ Angiulli and Pizzuti $[2002))] \ldots$. . . . . . 9

2.2 Dataset bidimensional (Inspirado em (Breunig et al., 2000)) . . . . . . . . . 11

2.3 Intuição básica de deteç̧ão de outliers baseada em ângulo (Inspirado em (Kriegel et al. 2008) $\ldots \ldots \ldots \ldots \ldots \ldots \ldots \ldots$

$4.1 \quad$ Resultados em algumas bases de dados comparando precision at $n(\underline{P @ n})$,

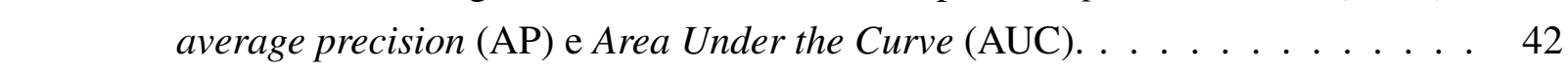

$4.2 \quad$ Ranking dos métodos pela performance considerando todos os valores de $k \mathrm{e}$ todas as bases de dados. . . . . . . . . . . . . . . . 44

4.3 Comparação dos melhores valores de AUC obtidos por método, considerando todas as bases de dados. . . . . . . . . . . . . . . 45

4.4 Comparação entre os melhores resultados alcançados por base de dados, considerando a versão normalizada e sem duplicatas. . . . . . . . . . . . . . . . 46

4.5 Diversidade dos rankings dos outliers em 17 bases de dados (sem duplicatas, normalizadas, aprox. 5\% outliers). As colunas estão relacionadas com os métodos nesta ordem: KNN, KNNW, LOF, SimplifiedLOF, LoOP, LDOF, ODIN, FastABOD, COF, KDEOS, LDF, INFLO. Note que FastABOD está faltando para ALOI, devido às exigências excessivas de recursos computacionais e LDF está faltando para InternetAds, devido à estimativas de densidade de kernel de

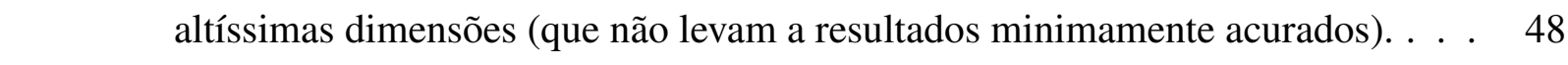

4.6 Comparação entre a diversidade de detecção dos verdadeiros outliers entre as quatro versões utilizadas para as bases WBC e PageBlocks (aprox. 5\% outliers): com/sem duplicatas e com/sem normalização. . . . . . . . . . . . . . . . . . . 49 
4.7 Dificuldade vs. Diversidade das bases de dados com 3\% a 5\% de outliers (sem duplicatas e normalizadas). Para cada base de dados com diferentes variantes de subamostragem, elipses com 95\% de confiança são apresentadas assim como os pontos individuais representando cada variante. As médias das elipses, que não correspondem a instâncias de dados reais, estão indicadas por um ' $X$ ' rotulada pela base de dados correspondente. Os valores dos rankings aleatórios são resultados de 100 simulações em uma base de dados com 200 instâncias, das quais 10 são outliers.

4.8 À esquerda: a distribuição dos valores observados de dificuldade para cada base de dados. À direita: para cada base de dados, a distribuição dos valores de dificuldade obtidos após permutar aleatoriamente os rótulos das instâncias. Para bases de dados com apenas uma variante entre 3\% e 5\% de outliers, apenas um único valor foi exibido. . . . . . . . . . . . . . . . . . . . 52

4.9 Novos valores de AUC aplicando técnicas de seleção de atributos em bases de dados sem duplicatas e sem normalização. . . . . . . . . . . . . . . . . . . 54

4.10 Novos valores de AUC aplicando técnicas de seleção de atributos em bases de dados sem duplicatas e com normalização. . . . . . . . . . . . . . . . . . . 55

4.11 Novos valores de AUC aplicando técnicas de seleção de atributos em bases de dados com duplicatas e com normalização. . . . . . . . . . . . . . . . . . . . . 55 


\section{Lista de Tabelas}

2.1 Ranking retornado de um algoritmo de detecção de outliers e os resultados das métricas de avaliação. Complementariedade das métricas $\mid \mathrm{AUC}] \mathrm{e}|P @ n|$ (utilizando $n=|O|)$. . . . . . . . . . . . . . . . . . 22

2.2 Ranking retornado de um algoritmo de detecção de outliers e os resultados das métricas de avaliação. Resultados enganosamente altos para as medidas $[P @ n \mid \mathrm{e}$ $\mathrm{AP}($ utilizando $n=|O|) . \ldots \ldots \ldots \ldots 22$

$3.1 \quad$ Bases de dados utilizadas por Emmott et al. (2013). . . . . . . . . . . . . 26

$4.1 \quad$ Bases coletadas com semântica. ． . . . . . . . . . . . . . . . . . . 34

4.2 Bases coletadas da literatura . . . . . . . . . . . . . . . . . . . . . 38

4.3 Relação entre bases utilizadas na literatura, artigos importantes na área e o préprocessamento realizado. . . . . . . . . . . . . . . . . . 40

A.1 Resultado da medida de avaliação AUC para bases de dados sem duplicatas e sem normalização. . . . . . . . . . . . . . . . . . . . . 60

A.2 Resultado da medida de avaliação AUC para bases de dados sem duplicatas e com normalização. . . . . . . . . . . . . . . . . . . . . 61

A.3 Resultado da medida de avaliação AUC para bases de dados com duplicatas e sem normalização. . . . . . . . . . . . . . . . . . . 62

A.4 Resultado da medida de avaliação AUC para bases de dados com duplicatas e com normalização. . . . . . . . . . . . . . . . . . . 62 



\section{Lista de Abreviaturas}

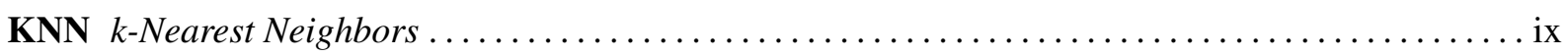

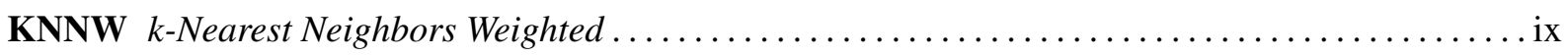

ODIN Outlier Detection using Indegree Number .....................................

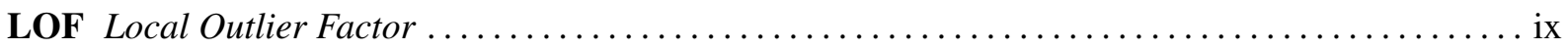

Simplified LOF Simplified Local Outlier Factor ..................................

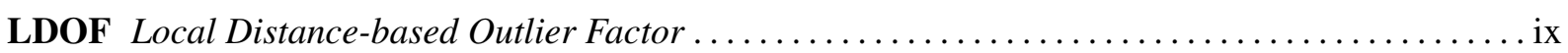

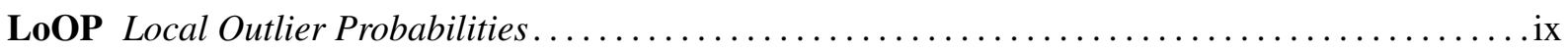

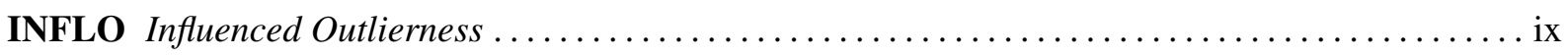

COF Connectivity-based Outlier Factor ..............................................

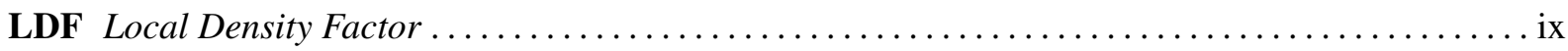

KDEOS Kernel Density Estimates Outlier Score ..................................

Fast ABOD Fast Angle-based Outlier Detection .....................................

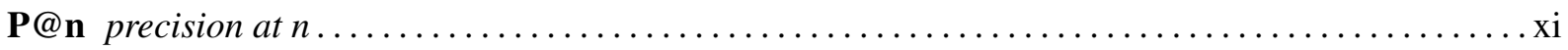

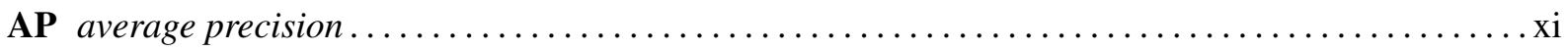

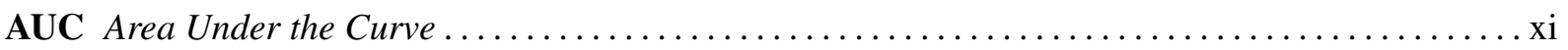

LOCI Local Correlation Integral.......................................... 3

PLOF Probabilistic Local Outlier Factor ................................... 14

RNN Reverse Nearest Neighbors ..........................................

NN Nearest Neighbors ................................................ 15 
IS Influence Space

KDE Kernel Density Estimation

LDE Local Density Estimation.

ABOD Angle-based Outlier Detection

ROC Receiver Operating Characteristic .

TPR True Positive Rate

FPR False Positive Rate.

IDF Inverse Document Frequency

KLR Kernel Logistic Regression

WBC Wisconsin Breast Cancer.

WDBC Wisconsin Diagnostic Breast Cancer.

ALOI Amsterdam Library of Object Images ...

HSB Hue-Saturation-Brightness

WPBC Wisconsin Prognostic Breast Cancer

RMS Root Mean Square 


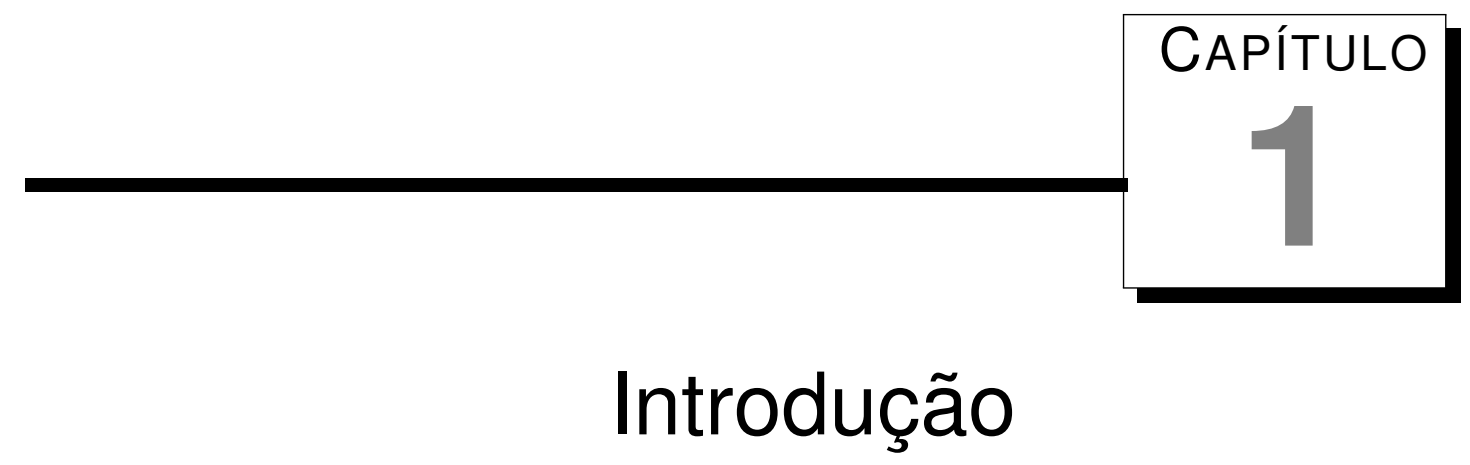

A tecnologia utilizada para desenvolvimentos na área da informática tem evoluído de forma extraordinária. As capacidades de processamento e armazenamento de sistemas de computadores têm aumentado ordens de grandeza durante as últimas décadas. Concomitantemente, a nossa capacidade de gerar e coletar dados de fontes variadas (por exemplo, a internet, satélites, redes de sensores, telescópios, sistemas de vigilância, etc.) aumentou proporcionalmente ou ainda mais rápido, assim propiciando um ambiente adequado para a aplicação de tecnologias emergentes de informação. A ampla utilização de código de barras de identificação de produtos, a informatização das transações comerciais e os avanços em dispositivos para coleta de dados, por exemplo, têm dado origem a bases de dados muito grandes em muitas áreas da atividade humana. Paradoxalmente, estes desenvolvimentos tecnológicos têm causado um grande problema de superabundância de dados, na medida em que nossa capacidade de coletar e armazenar dados já ultrapassou em muito nossa capacidade humana para analisar e extrair conhecimento a partir deles (Fayyad et al., 1996). Nesse contexto, técnicas avançadas capazes de sumarizar, processar e transformar dados em conhecimento útil de uma forma inteligente e automatizada tornam-se cada vez mais essenciais. Por esta razão, pesquisadores de diversas áreas têm se empenhado no estudo de métodos para o que tem sido chamado de mineração de dados ou data mining.

O termo mineração de dados (Fayyad et al., 1996; Bigus, 1996, Witten and Frank, 2005, Han and Kamber, 2006) refere-se à extração (ou mineração) de conhecimento em grandes conjuntos de dados através de técnicas estatísticas e computacionais. Existem várias maneiras de extrair tais conhecimentos úteis de grandes quantidades de dados, de acordo com o objetivo a ser alcançado. Agrupar dados, classificar instâncias, analisar associações, são exemplos de subáreas incluídas em mineração de dados. Uma tarefa que possui um papel fundamental na descoberta de padrões é a detecção de outliers. Conseguir determinar se uma dada instância é apenas um ruído que se deseja eliminar em uma etapa de pré-processamento, ou se esta mesma instância possui um padrão nunca analisado antes, a qual possa representar um comportamento 
extraordinário e, portanto, passível de estudo e atenção especial, é uma tarefa relevante para a área de mineração de dados. Por exemplo, detectar tais padrões pode estar associado a fraudes em transações bancárias, intrusões em redes de computadores, doenças incomuns, etc.

Como a área de detecção de anomalias está atualmente atraindo a atenção de vários pesquisadores, diversas técnicas para detectar outliers estão surgindo. Cada técnica é construída sobre uma certa perspectiva para detectar as anomalias presentes em uma base de dados. Por isso, tornou-se comum a distinção entre técnicas de detecção de anomalias globais e locais (Kriegel et al., 2009b; Zimek et al., 2012), em que ambas se referem ao escopo dos dados a ser considerado quando o método decide se (ou o grau em que) uma instância deve ser considerada um outlier. Outra diferença que existe entre as técnicas é relacionada à resposta produzida: a técnica pode retornar o conjunto de dados rotulados de forma binária entre outliers ou não outliers (inliers), ou retornar uma pontuação (scoring ou ranking) para cada instância, determinando o seu grau ou probabilidade de ser um outlier. Uma terceira diferença entre técnicas de detecção de anomalias está relacionada ao conhecimento ou ausência de conhecimento a priori de um conjunto de instâncias inliers e de um conjunto de instâncias outliers antes de executá-las. Se este conhecimento antecipado sobre um conjunto de instâncias está disponível para as técnicas de detecção de anomalias, tal técnica trabalha de uma maneira supervisionada. Caso contrário, a técnica não possui nenhum conhecimento prévio sobre instâncias que de fato são ou não outliers e a mesma trabalha de maneira não supervisionada. O presente trabalho se concentra nas técnicas de detecção não supervisionadas.

De maneira geral, um outlier no contexto de detecção não supervisionada pode ser descrito como "an observation (or subset of observations) which appears to be inconsistent with the remainder of that set of data" (Barnett and Lewis, 1994). Uma das definições mais amplamente citada é a de Hawkins (1980), que descreve um outlier como "an observation that deviates so much from other observations as to arouse suspicion that it was generated by a different mechanism". A maneira pela qual uma observação é julgada inconsistente ou suspeita não é definida de maneira geral, ao contrário, depende do cenário de aplicação e do método de detecção a ser utilizado. Sob esta ótica, nota-se então que esta área envolve um tipo de subjetividade que também está presente, por exemplo, em agrupamento não supervisionado de dados (data clustering) (Jain and Dubes, 1988; Gan et al., 2007).

Uma parte importante dos trabalhos existentes em detecção não supervisionada de outliers está concentrada no campo da estatística (Hawkins, 1980, Barnett and Lewis, 1994), na qual este tema tem sido investigado por várias décadas. Existem dezenas de testes estatísticos que podem ser aplicados para detectar discordâncias/anomalias em diferentes cenários de aplicação (Barnett and Lewis, 1994). Tais testes diferem na hipótese sobre o tipo de distribuição dos dados, na hipótese sobre o tipo dos dados propriamente ditos, na natureza dos outliers esperados, dentre outros. Eles possuem em comum, entretanto, o fato de possuírem ao menos uma das seguintes limitações: (i) em sua maioria os testes se limitam a dados numéricos unidimensionais ou descritos por um número pequeno de dimensões. Esta limitação os torna inadequados para a maioria das aplicações reais em bases de dados heterogêneas e multidimensionais; e (ii) em sua 
maioria os testes são paramétricos, ou seja, são baseados na hipótese que os dados seguem uma determinada distribuição cujos parâmetros podem ou não ser conhecidos, dependendo do teste. Por exemplo, uma regra prática (Howell, 1998) é tomar como outliers observações que desviam mais do que 3 vezes o desvio padrão a partir da média de uma distribuição Normal (unidimensional). No caso multidimensional, limiares equivalentes podem ser estabelecidos e impostos à distância de Mahalanobis. Uma limitação desta abordagem é que os parâmetros da distribuição em geral não são conhecidos e precisam portanto ser estimados a partir dos próprios dados, entretanto tal estimação é geralmente sensível e distorcida pela presença dos próprios outliers que se deseja detectar. Logo, extensivos testes podem ser requeridos para tentar encontrar uma distribuição que se adapte aos dados quando possível.

Em razão das limitações discutidas acima, métodos alternativos de detecção de outliers passaram a ser desenvolvidos que (i) não se baseiam em hipóteses específicas sobre a distribuição dos dados (métodos não paramétricos) e (ii) são computacionalmente viáveis para aplicação em bases de dados de forma mais ampla, possivelmente de grande porte e multidimensionais. Esta vertente ganhou impulso a partir dos trabalhos pioneiros de Knorr and Ng (1998) e Knorr et al. (2000), em que se introduz técnicas não paramétricas computacionalmente escaláveis de detecção de outliers em grandes bases de dados ao mesmo tempo em que se generaliza e unifica abordagens estatísticas paramétricas quando certas hipóteses são satisfeitas. Várias novas abordagens surgiram e ainda têm surgido a partir deste trabalho (Orair et al., 2010), tais como os algoritmos KNN (Ramaswamy et al., 2000), KNNW (Angiulli and Pizzuti, 2002), LOF (Breunig et al., 2000), LDOF (Zhang et al., 2009), COF (Tang et al., 2002), INFLO (Jin et al., 2006), LoOP (Kriegel et al., 2009a), Local Correlation Integral (LOCI) (Papadimitriou et al., 2003), dentre diversos outros. Cada um desses algoritmos, entretanto, utiliza um critério ou viés indutivo próprio para julgar de forma quantitativa o nível de aderência de cada instância com o conceito de outlier, que é naturalmente subjetivo no contexto não supervisionado. Isso torna a escolha de um algoritmo em particular uma tarefa não trivial em aplicações práticas. Embora seja de conhecimento comum que nenhum algoritmo de aprendizado de máquina pode ser superior a todos os demais em todos os cenários de aplicação, é uma questão relevante se o desempenho de certos algoritmos em geral tende a dominar o de determinados outros, ao menos em classes particulares de problemas.

O procedimento de avaliação de algoritmos de detecção de outliers usualmente adotado na literatura é baseado na utilização de bases de dados previamente rotuladas, nas quais os outliers (segundo alguma intuição particular) são previamente conhecidos. No caso dos algoritmos não supervisionados, os rótulos não são utilizados pelo algoritmo em si, mas para avaliar os resultados produzidos por este. Especificamente, a categorização binária ou o ranking das observações produzidas pelo algoritmo são comparados com a rotulação considerada correta e previamente conhecida (ground truth). O resultado quantitativo desta comparação, por exemplo utilizando acurácia ou área sob a curva ROC (Schubert et al., 2012), é tomado como medida de qualidade do algoritmo de detecção em questão. Porém, o procedimento de avaliação descrito acima não é viável para a avaliação da qualidade dos resultados em aplicações práticas, 
pois a disponibilidade de uma base de dados pré-rotulada é obviamente inconsistente com a hipótese fundamental do aprendizado não supervisionado. Entretanto, esse mesmo procedimento de avaliação é perfeitamente válido para a análise comparativa entre diferentes algoritmos de detecção de outliers disponíveis na literatura. Contudo, ao contrário de outras áreas, onde coleções de bases de dados estabelecidas como benchmarks permitem padronizar o procedimento de avaliação do desempenho de novos algoritmos, os procedimentos de avaliação na literatura de detecção não supervisionada de outliers têm sido até então essencialmente ad hoc. De fato, ao serem propostos, usualmente os algoritmos nesta área mostram desempenhos melhores do que algum subconjunto de outros algoritmos existentes apenas no que diz respeito a uma coleção pequena de bases de dados, em geral escolhidas arbitrariamente e nas quais, muitas vezes, a própria rotulação dos supostos outliers pode ser questionável. Isso torna particularmente difícil discernir aqueles algoritmos que de fato representam algum avanço no estado da arte.

Seguindo essa linha de conhecimento apresentada anteriormente, o trabalho em questão procura contribuir com o estudo, seleção e pré-processamento de bases de dados que sejam apropriadas para se juntar a uma coleção benchmark de bases de dados para detecção não supervisionada de outliers. Adicionalmente, avalia-se comparativamente o desempenho de algoritmos de detecção de outliers nas bases de dados de interesse.

\subsection{Objetivos}

Este trabalho de mestrado tem como objetivo estudar, avaliar e comparar técnicas de detecção não supervisionada de outliers. Para possibilitar esse estudo, quatro passos foram seguidos e investigados cuidadosamente:

- Criação de uma coleção benchmark de bases de dados para detecção de outliers.

- Análise dos algoritmos de detecção de outliers e suas respectivas parametrizações utilizadas no estudo comparativo.

- Consolidação das estatísticas de interesse coletadas com a execução dos algoritmos, possibilitando a comparação entre eles.

- Análise crítica dos resultados obtidos, verificando a qualidade da coleção benchmark de bases de dados e dos algoritmos de detecção de anomalias selecionados.

Para alcançar tais objetivos, primeiramente uma coleção benchmark de bases de dados para a detecção de outliers foi criada. Posteriormente, os algoritmos selecionados foram aplicados e as estatísticas de interesse coletadas. Após isto, foi possível obter uma conclusão crítica a respeito dos métodos de detecção de outliers e das bases de dados selecionadas para compor o benchmark. 


\subsection{Organização do Trabalho}

O presente trabalho está organizado da seguinte maneira:

Capítulo 2. São apresentadas técnicas de detecção não supervisionada de outliers. Diferenças entre técnicas de detecção global e local de outliers são enfatizadas.

Capítulo 3 Aborda-se a dificuldade de se criar uma boa coleção benchmark de bases de dados para detecção de outliers.

Capítulo 4: Resultados relacionados ao benchmark de bases de dados e aos algoritmos de detecção de outliers são apresentados e analisados.

Capítulo 5. Apresenta-se a conclusão do trabalho. 



\section{Detecção Não Supervisionada de Outliers}

Nos últimos anos, a área de detecção de anomalias vem ganhando cada vez mais atenção e atraindo mais pesquisadores. Isso porque, detectar outliers é uma tarefa importante em diversas áreas de aplicação no mundo real, como detecção de tumores cerebrais (Prastawa et al., 2004), detecção de intrusão em redes (Wang and Stolfo, 2004; Zhang and Zulkernine, 2006), detecção de incontinência urinária feminina através de dados médicos (Laurikkala et al., 2000), entre outras. Para que isso fosse possível, algoritmos pioneiros nessa área foram desenvolvidos como por exemplo em Ramaswamy et al. (2000). Grande parte dos algoritmos desenvolvidos não afirmam se uma instância é um outlier ou não, e sim atribuem valores associados ao grau em que tal instância se caracteriza como outlier.

Os algoritmos para detecção de outliers propostos inicialmente tinham como objetivo encontrar anomalias em um contexto global (Knorr and Ng, 1998; Ramaswamy et al., 2000), em que todas as instâncias são tratadas da mesma forma, independente da região do espaço de atributos em que se encontram, o que em muitos casos pode gerar problemas na presença de outliers em regiões do espaço com diferentes densidades de dados. Para contornar tal situação, algoritmos de detecção local de outliers foram desenvolvidos a partir do trabalho pioneiro de Breunig et al. (2000). Existe portanto uma distinção entre algoritmos de detecção global e local de anomalias no que diz respeito ao escopo da base de dados em busca do score de outlier para cada observação. Os primeiros assumem que apenas um mecanismo gerou todos os dados, não importando a localização do ponto! que está sendo observado em questão. Já algoritmos de detecção local consideram que não há um número exato de mecanismos que geraram os dados,

\footnotetext{
${ }^{1}$ Ponto é uma instância presente em uma base de dados, denominação esta que advém (mas não se limita aqui neste trabalho) do cenário em que as instâncias são descritas apenas por atributos numéricos e portanto podem ser vistas como um ponto no espaço cartesiano.
} 
analisando o comportamento da vizinhança ao redor de um ponto.

Neste capítulo é realizado um estudo dos algoritmos de detecção de outliers globais e locais mais conhecidos na literatura e que serão utilizados no decorrer de todo o projeto.

\subsection{Algoritmos de Detecção de Outliers Globais}

\subsubsection{KNN}

O algoritmo KNN (Ramaswamy et al., 2000) é um dos mais simples e eficientes algoritmos para detecção de anomalias encontrados na literatura. Ramaswamy et al. (2000) definiram a distância entre um ponto $p$ para o seu $k$-ésimo vizinho mais próximo, definido por $D^{k}(p)$, como o score final produzido pelo algoritmo. Assim, a medida que um ponto está mais distante do seu $k$-ésimo vizinho, maior o seu potencial de ser um outlier.

Definição 1. Dado um conjunto de dados com $N$ pontos e os parâmetros $n$ e $k$, um ponto p é um $D_{n}^{k}$ outlier se não há mais do que $n-1$ outros pontos $p^{\prime}$ para os quais $D^{k}\left(p^{\prime}\right)>D^{k}(p)$.

Formalmente, o KNN possui a definição acima, que resulta em uma classificação binária dos dados em outliers e inliers 2 . Porém, não é obrigatório fornecer a quantidade de outliers desejados (parâmetro $n$ ). De fato, o algoritmo necessita apenas da quantidade $k$ de vizinhos mais próximos a um ponto $p$ para executar e obter scores de saída para cada instância, ao invés de uma classificação binária.

Apesar de ser simples, o algoritmo proposto por Ramaswamy et al. (2000) é bastante utilizado tanto para resultados práticos como base para novas ideias. Esta técnica foi selecionada para o trabalho devido à sua simplicidade, popularidade e sucesso perante a uma grande gama de experimentos na literatura.

\subsubsection{KNNW}

Criado a partir do algoritmo KNN proposto por Ramaswamy et al. (2000), o KNNW, ou KNN ponderado, foi proposto por Angiulli and Pizzuti (2002). A mesma ideia de utilizar vizinhos mais próximos para definir o score da instância é utilizada por este algoritmo. Porém, ao invés de utilizar apenas a distância de um ponto $p$ até o $k$-ésimo vizinho mais próximo, o KNNW utiliza a distância de todos os vizinhos mais próximos até o $k$-ésimo vizinho. Essa ideia evita que dois pontos que possuem uma distância $d$ para o seu $k$-ésimo vizinho mais próximo possuam o mesmo score final, mesmo que um desses pontos seja mais denso do que o outro.

A Figura 2.1 é um caso interessante, no qual os scores do KNN para os pontos $p_{1}$ e $p_{2}$ são idênticos, dado que a distância de $p_{1}$ até $q_{1}$ é a mesma de $p_{2}$ até $q_{2}$. Porém, é intuitivo na Figura 2.1 que $p_{1}$ deve ser mais outlier do que $p_{2}$, pois sua vizinhança é mais esparsa. Para resolver esse problema, o KNNW utiliza a distância somada de um ponto para todos os seus vizinhos mais próximos até um $k$-ésimo vizinho mais próximo. Os autores desta técnica explicam a fundo

\footnotetext{
${ }^{2}$ Inliers são instâncias consideradas normais dentro do padrão da base de dados, tipicamente parte de um cluster.
} 

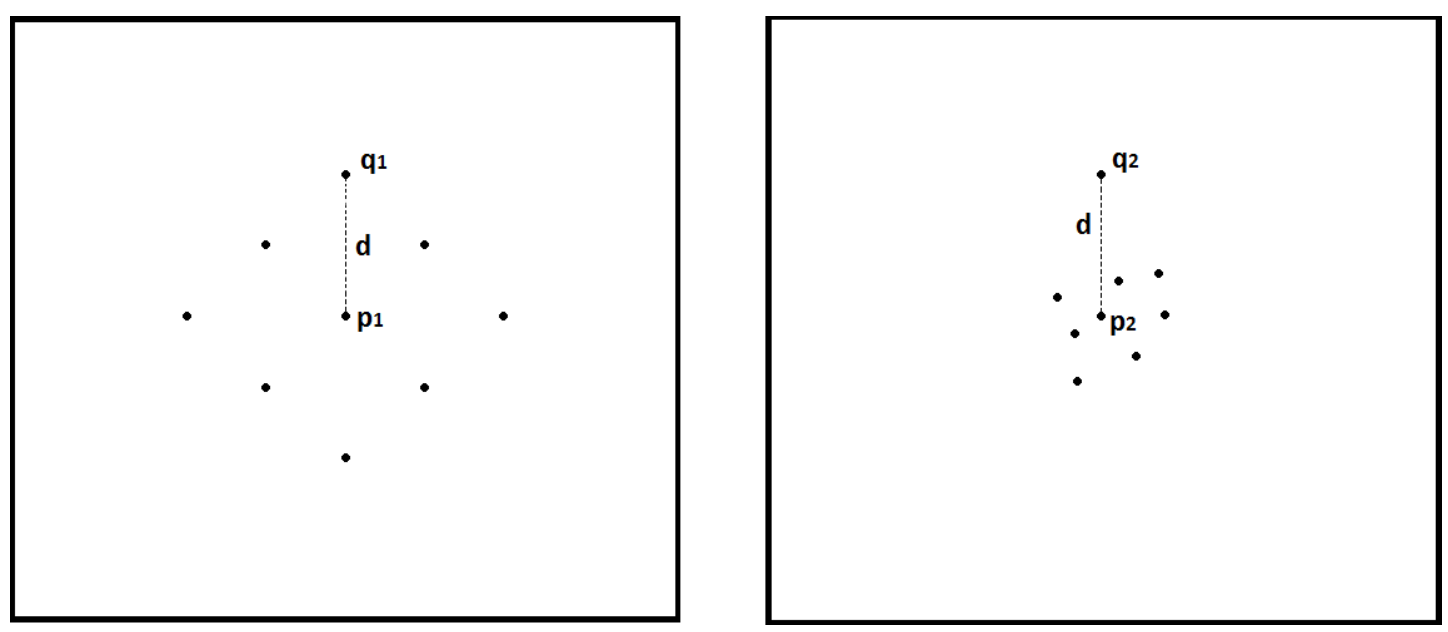

Figura 2.1: Dois pontos pertencentes a regiões de densidades distintas, porém com o mesmo valor de $D^{k}(k=8)$ (Inspirado em (Angiulli and Pizzuti, 2002))

cada propriedade do KNNW, entretanto são duas as definições que nos permitem entender o funcionamento do algoritmo.

Definição 2. Seja DB uma base de dados de dimensão $d, k$ um parâmetro e $p$ um ponto em DB. O peso de p em $\boldsymbol{D B}$ é definido como $w_{k}(p)=\sum_{i=1}^{k} d\left(p, n n_{i}(p)\right)$, onde $n n_{i}(p)$ é o i-ésimo vizinho mais próximo de p em $\boldsymbol{D B}$, e d $\left(p, n n_{i}(p)\right)$ é a distância entre p e o seu i-ésimo vizinho mais próximo.

Definição 3. Dado uma base de dados $\boldsymbol{D B}$, parâmetros $k$ e n, um ponto $p \in \boldsymbol{D B}$ é o n-ésimo outlier com respeito a $k$, escrito como outlier $r_{k}^{n}$, se existem exatos $n-1$ pontos $q$ em DB os quais $w_{k}(q)>w_{k}(p)$.

Assim como no algoritmo KNN no KNNW não é necessário o parâmetro $n$ para obter scores para todas as instâncias da base, dados pelos próprios pesos na Definição 2. Ambos os algoritmos possuem uma perspectiva de detecção global de outliers, que depende neste caso apenas da quantidade de vizinhos mais próximos a ser considerada, independente das possíveis diferenças entre as características (e.g. distribuição de probabilidade) dos dados em diferentes regiões do espaço.

\subsubsection{ODIN}

Este algoritmo proposto por Hautamaki et al. (2004) é um modelo baseado em grafos. Cada vértice do grafo é definido como outlier ou inlier através dos graus de entrada. Estes graus fazem referência à quantidade de vezes que um objeto pertence ao conjunto $\mathrm{KNN}$ de outros objetos. A intuição aqui é que os outliers ocorreriam com menos frequência como vizinhos mais próximos de outros pontos.

Definição 4. Dado um grafo KNN $G$ de uma base de dados $D B$, outlier é o vértice cujos graus de entrada são menores ou iguais a $T$. 
Um grafo KNN é definido como um grafo direcionado ponderado, no qual cada vértice representa um único ponto, e as arestas correspondem aos indicadores de pontos vizinhos. Cada vértice possui exatamente $k$ arestas para os $k$ pontos mais próximos de acordo com uma dada função de distância. O peso da aresta é a distância entre os pontos que formam a aresta.

A Definição 4 utilizada por Hautamaki et al. (2004) cita um threshold (T) para realizar a divisão binária entre outliers e inliers. Porém, apenas através da quantidade de graus de entrada de um vértice já é possível obter um ranking de todos os vértices e, consequentemente, o resultado final do algoritmo, que disponibiliza estes graus como scores de outliers. Contudo, é importante mencionar que nesta técnica, quanto menor a quantidade de arestas de entrada de entrada em um vértice, maior o seu grau de outlier.

\subsection{Algoritmos de Detecção de Outliers Locais}

\subsubsection{LOF}

Proposto por (Breunig et al., 2000), o algoritmo LOF (Local Outlier Factor) busca por outliers considerando a densidade de um ponto $p$ e a densidade de seus $k$ vizinhos. Essa técnica de detecção de anomalias foi pioneira tratando-se de detecção local. A partir deste algoritmo, vários outros surgiram, alguns dos quais serão utilizados no projeto e discutidos nas subseções seguintes.

Um exemplo utilizado por Breunig et al. (2000) para mostrar a ineficiência de algoritmos de detecção global de outliers em alguns casos está representada na Figura 2.2. Através desta figura, é possível perceber claramente que existem dois clusters $C_{1}$ e $C_{2}$ com uma variação de densidade muito grande entre eles. $\mathrm{O}$ cluster $C_{2}$ é bem mais denso e compacto do que o cluster $C_{1}$. Existe um outlier evidente, $o_{1}$, que pode ser facilmente detectado por um algoritmo de detecção de anomalias global, e uma instância $o_{2}$ que pode ser considerada outlier porque desvia um pouco do padrão apresentado pelo cluster $C_{2}$. As distâncias do outlier $o_{2}$ para instâncias do cluster $C_{2}$, em parte, são menores do que distâncias entre instâncias internas do cluster $C_{1}$. Portanto, caso um algoritmo de detecção global de outliers considere que $o_{2}$ é um outlier, várias instâncias internas ao cluster $C_{1}$ também poderão ser consideradas outliers, o que é contra-intuitivo.

Para resolver o problema citado acima, Breunig et al. (2000) desenvolveram o LOF, algoritmo de detecção local de anomalias que analisa as densidades relativas entre um ponto $p$ e sua vizinhança. Para obter a formulação final do cálculo de score para cada instância, algumas definições são necessárias.

Definição 5. Seja $D$ uma base de dados. Para qualquer inteiro positivo $k$, a $k$ _distance de um objeto $p$, definida como $k \_d i s t a n c e(p)$, é a distância $d(p, o)$ entre $p$ e um objeto o $\in D$ tal que: (i) no mínimo $k$ objetos $o^{\prime} \in D \backslash\{p\}$ têm-se $d\left(p, o^{\prime}\right) \leq d(p, o)$ e (ii) no máximo $k$ - 1 objetos $o^{\prime}$ $\in D \backslash\{p\}$ têm-se $d\left(p, o^{\prime}\right)<d(p, o)$. 


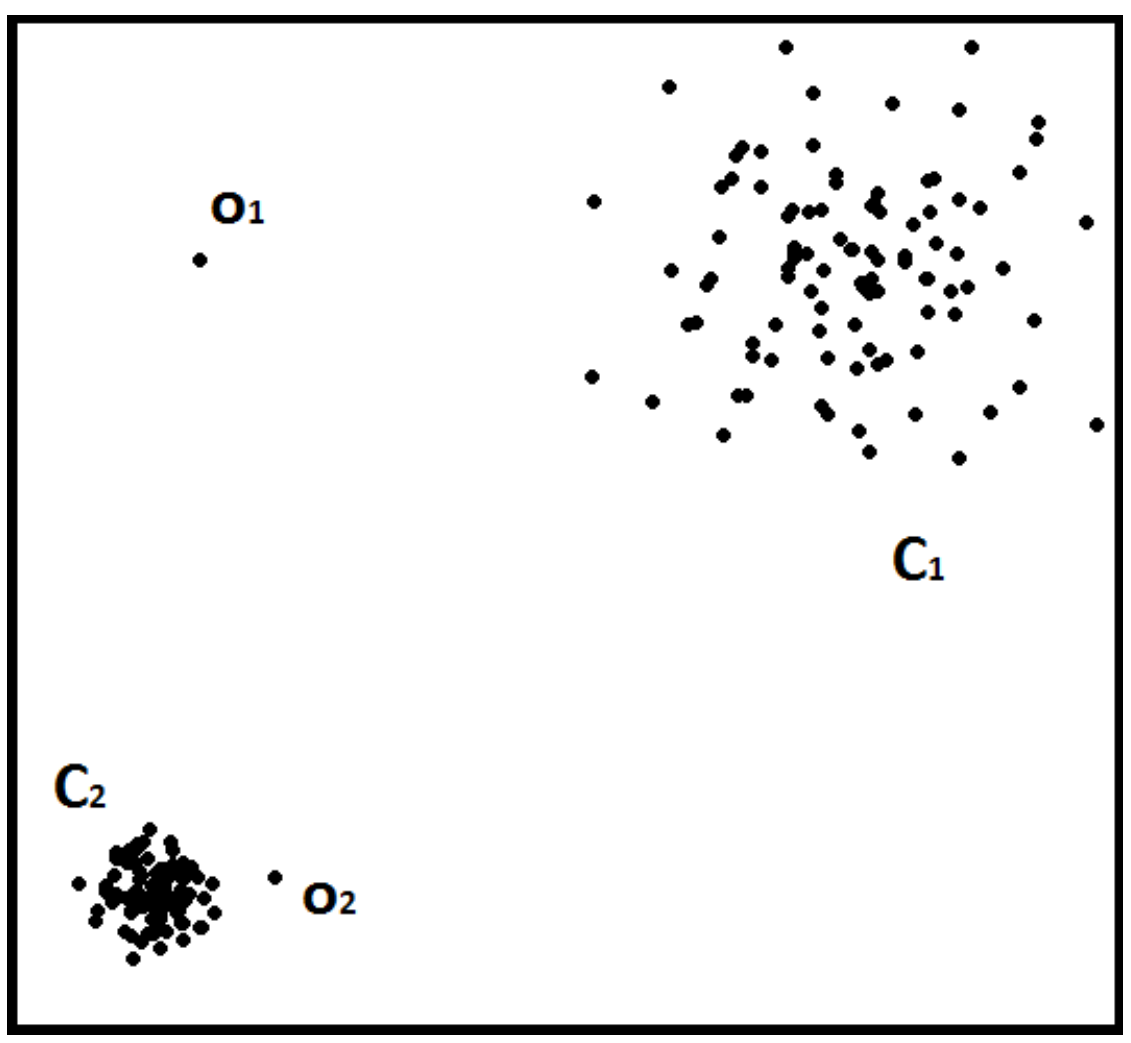

Figura 2.2: Dataset bidimensional (Inspirado em (Breunig et al., 2000)

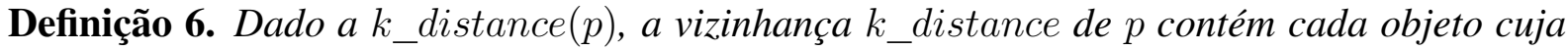
distância para p não é maior que $k \_d i s t a n c e(p)$. Formalmente, $N_{k \_d i s t ~}(p)=\{q \in D \backslash\{p\} \mid$ $d(p, q) \leq k \_$distance $\left.(p)\right\}$.

Definição 7. Seja k um número natural, a distância de alcançabilidade (ou "reachability distance”) de um objeto p para com um objeto o é definida como reach_dist $t_{k}(p, o)=$ max \{ $k \_$distance $\left.(o), d(p, o)\right\}$.

Este conceito de distância de alcançabilidade na Definição 7 foi utilizado para verificar qual é o limite da vizinhança de um ponto, com a ideia de eliminar a influência de outros outliers.

Com as definições apresentadas, o próximo passo é calcular a densidade da vizinhança de um ponto $p$. A Equação (2.1) é definida pelo inverso da média das distâncias de alcançabilidade entre $p$ e seus vizinhos. Quanto maior o valor de $\operatorname{lr} d_{k}(p)$, mais densa é a região onde se encontra $p$. O score final do algoritmo LOF é dado pela Equação (2.2), a qual calcula uma média das razões entre as densidades de cada vizinho mais próximo de $p$ em relação à densidade de $p$. Este cálculo possibilita verificar se a vizinhança mais próxima de $p$ é relativamente mais densa do que o próprio $p$ ou não, evitando os problemas de algoritmos globais como citados anteriormente.

$$
\begin{gathered}
\operatorname{lrd}_{k}(p)=1 /\left(\frac{\sum_{o \in N_{k \_d i s t}(p)} \text { reach_dist }(p, o)}{\left|N_{k \_d i s t}(p)\right|}\right) . \\
\operatorname{LOF}_{k}(p)=\frac{\sum_{o \in N_{k \_d i s t}(p)} \frac{\operatorname{lrd} d_{k}(o)}{\mid r d_{k}(p)}}{\left|N_{k \_d i s t}(p)\right|} .
\end{gathered}
$$


Assim como apresentado nos dois algoritmos de detecção global de anomalias, LOF também utiliza apenas o parâmetro $k$ para realizar seus cálculos. Os autores chamam esse parâmetro de MinPts, porém aqui foi definido como $k$, que seria a quantidade de vizinhos mais próximos a um ponto $p$. O score produzido por $\mathrm{LOF}$ possui valor esperado em torno de 1 quando a densidade do ponto e de seus vizinhos é aproximadamente a mesma, o que ocorre para os inliers internos a um cluster com distribuição uniforme. Espera-se valores cada vez maiores que 1 quanto mais um ponto se caracteriza como outlier. Os autores também discutem a possibilidade da resposta do algoritmo ser infinita, quando a base de dados possui objetos duplicados e, como alternativa, sugerem atribuir ao grupo de $k$ vizinhos mais próximos apenas aqueles diferentes de um ponto $p$ observado.

\subsubsection{Simplified LOF}

Este método é uma variante bem próxima ao algoritmo proposto por Breunig et al. (2000). A única diferença entre esta variante e o algoritmo original LOF é a alteração da distância de alcançabilidade entre $p$ e seus vizinhos para uma simples estimativa de densidade, utilizando a distância até o $k$ vizinho mais próximo ( $\left.k \_d i s t\right)$. Ao invés de utilizar a fórmula descrita pela Equação (2.1), esta variante utiliza a Equação (2.3).

$$
\operatorname{dens}(p)=\frac{1}{k \_\operatorname{dist}(p)} .
$$

O algoritmo Simplified LOF foi definido somente em Schubert et al. (2014b), e será utilizado nos testes comparativos realizados neste trabalho.

\subsubsection{LDOF}

A ideia do LDOF consiste em medir o grau em que um objeto desvia da sua vizinhança, ao invés de trabalhar com distâncias globais ou densidades locais (Zhang et al., 2009). Esta técnica possui uma conceituação simples, na qual duas definições de distâncias são necessárias e, por fim, a equação de score final do algoritmo.

Definição 8. Seja $N_{p}$ o conjunto de k vizinhos mais próximos (ou "k-nearest neighbors", "KNN") de um objeto $x_{p}$ (excluindo $x_{p}$ ). A distância $K N N$ de $x_{p}$ é definida aqui como a média das distâncias de $x_{p}$ para todos os objetos em $N_{p}$. Mais formalmente, seja dist $\left(x, x^{\prime}\right) \geq 0$ a medida de distância entre os objetos $x$ e $x^{\prime}$. A distância KNN de um objeto $x_{p}$ é definida aqui como

$$
\bar{d}_{x_{p}}:=\frac{1}{k} \sum_{x_{i} \in N_{p}} \operatorname{dist}\left(x_{i}, x_{p}\right) .
$$

Definição 9. Dado um conjunto de $k$ vizinhos mais próximos $\left(N_{p}\right)$ de um objeto $x_{p}$, a distância média entre os vizinhos mais próximos de $x_{p}$ é definida como a média das distâncias entre os objetos em $N_{p}$ :

$$
\bar{D}_{x_{p}}:=\frac{1}{k(k-1)} \sum_{x_{i}, x_{i^{\prime}} \in N_{p}, i \neq i^{\prime}} \operatorname{dist}\left(x_{i}, x_{i^{\prime}}\right)
$$


Com essas definições, o valor de LDOF para cada objeto da base é calculado realizando uma divisão dessas duas distâncias. Resumindo, o score produzido pelo algoritmo para um ponto $p$ é a divisão entre a distância média do ponto $p$ para com seus $k$ vizinhos e a distância média entre os próprios vizinhos de $p$. Esse score está representado na Equação (2.4).

$$
\operatorname{LDOF}_{k}\left(x_{p}\right):=\frac{\bar{d}_{x_{p}}}{\bar{D}_{x_{p}}} .
$$

Segundo Zhang et al. (2009), quando o valor de LDOF for menor ou próximo de 1 significa que $x_{p}$ está cercado por uma nuvem de dados. Por outro lado, caso LDOF seja bem acima de $1, x_{p}$ está fora dessa nuvem de dados. Com isso, é trivial dizer que quanto maior o valor de LDOF para um ponto $x_{p}$, mais distante o ponto é do sistema/comportamento apresentado pela sua vizinhança.

Para realizar os cálculos desse algoritmo, apenas o valor de $k$ é necessário, assim como nos algoritmos anteriormente apresentados.

\subsubsection{LOOP}

Os algoritmos vistos até o momento geram como saída scores que não possuem um valor máximo previamente definido. Outro ponto importante é que nesses algoritmos não é possível, apenas com os valores de saída, determinar com embasamento teórico se um ponto é um outlier ou não de forma binária (e.g. quão maior que 1 deve ser um score de LOF ou LDOF para caracterizar um outlier?). Portanto, surgiu a ideia proposta por Kriegel et al. (2009a) de criar um algoritmo para gerar valores probabilísticos para cada objeto da base de dados, informando qual a chance de um dado ponto ser considerado outlier ou inlier, o que possui uma interpretação absoluta e não apenas relativa. O LoOP foi idealizado com base em conceitos estatísticos e possui uma formulação mais complexa do que os algoritmos apresentados até o presente momento.

$\mathrm{Na}$ estatística é comum declarar um ponto outlier quando o mesmo desvia mais do que uma quantidade $\lambda$ de vezes o desvio padrão $\sigma$ da média. Assim como citado em Kriegel et al. (2009a), os valores de $\lambda$ são tipicamente aqueles da regra empírica "three sigma", onde $\lambda=$ $1 \Leftrightarrow \varphi \approx 68 \%, \lambda=2 \Leftrightarrow \varphi \approx 95 \%$ e $\lambda=3 \Leftrightarrow \varphi \approx 99.7 \%$.

Considerando uma base de dados $\mathrm{D}$, o primeiro passo dado pelos autores foi a definição de uma distância probabilística de $o \in D$ para elementos pertencentes ao conjunto $S \subseteq D$ $(p \operatorname{dist}(o, S)$ ), descrito pela Equação 2.5), em que $d(o, s)$ é a distância entre $o$ e um elemento do conjunto S. Intuitivamente, uma esfera em torno do objeto o com raio pdist cobre qualquer elemento do conjunto $S$ com probabilidade $\varphi$ (Kriegel et al. 2009a). O próximo passo dado pelos autores foi calcular uma distância padrão (standard distance) entre os objetos em $S$ para $o$, similar ao desvio padrão. Para isso, foi assumido que $o$ é o centro de $S$ e um conjunto de distâncias de $s \in S$ para $o$ é aproximadamente meia Gaussiana (Kriegel et al., 2009a). A distância padrão está descrita na Equação (2.6).

$$
\forall s \in S: P[d(o, s) \leq \operatorname{pdist}(o, S)] \geq \varphi
$$




$$
\sigma(o, S)=\sqrt{\frac{\sum_{s \in S} d(o, s)^{2}}{|S|}} .
$$

Portanto, de acordo com as fórmulas acima e com a definição de $\lambda$ provinda da estatística, a distância de $o$ para um conjunto de pontos $S$ pode ser definida pela Equação (2.7), em que $\operatorname{pdist}(\lambda, o, S)$ é o conjunto de distâncias probabilísticas entre um ponto $o$ e um conjunto $S$. Segundo Kriegel et al. (2009a), este conjunto de distâncias probabilísticas estimam a densidade ao redor do objeto $o$ baseado no conjunto $S$. O parâmetro $\lambda$ permite alterar a proximidade da densidade, porém não influencia nos rankings dos outliers.

$$
\operatorname{pdist}(\lambda, o, S):=\lambda \cdot \sigma(o, S)
$$

Com a finalidade de obter o conjunto $S$ de pontos em relação a um ponto $o$, os autores utilizaram a busca através dos $k$ vizinhos mais próximos de $o$. Através desse conjunto de distâncias probabilísticas entre um ponto e um conjunto de pontos vizinhos $(k)$, é possível calcular uma razão da estimativa de densidade ao redor de um ponto $o$ e a estimativa esperada da densidade ao redor de todos os vizinhos de $o$. Com isso, surgiu o chamado fator de outlier probabilístico local (Probabilistic Local Outlier Factor ( $(\underline{\mathrm{PLOF}})$ ) descrito pela Equação (2.8). De acordo com Kriegel et al. (2009a), o PLOF de um objeto $o \in D$ calcula a proporção da estimativa de densidade em torno de $o$, o qual é baseado em sua vizinhança $(S(o)$ ), e o valor esperado das estimativas de densidades em torno de todos os objetos no conjunto $S(o)$.

$$
P L O F_{\lambda, S(o)}:=\frac{\operatorname{pdist}(\lambda, o, S(o))}{E_{s \in S(o)}[\operatorname{pdist}(\lambda, s, S(s))]}-1
$$

Objetivando alcançar uma normalização, tornando o dimensionamento de PLOF independente da distribuição particular dos dados, uma nova variável $n$ PLOF foi utilizada como descrito na Equação (2.9). Esse valor pode ser visto como o desvio padrão dos valores de PLOF, assumindo o valor de média 0 (Kriegel et al., 2009a).

$$
n \underline{\mathrm{PLOF}}:=\lambda \cdot \sqrt{E\left[(\overline{\mathrm{PLOF}})^{2}\right]} .
$$

Por fim, para transformar os valores PLOF em probabilidades, os autores assumiram que os valores estão normalmente distribuídos em torno de 1 com um desvio padrão de $n$ PLOF Com isso foi aplicado a Função Erro Gaussiana (Gaussian Error Function) para obter o valor de probabilidade LoOP de um ponto $o \in D$ ser um outlier (Kriegel et al., 2009a). O score final produzido por este algoritmo está descrito pela Equação (2.10), onde erf é a Função Erro Gaussiana.

$$
L o O P_{s(o)}:=\max \left\{0, \operatorname{erf}\left(\frac{\underline{\mathrm{PLOF}}_{, S(o)}}{n \sqrt{\mathrm{PLOF}} \sqrt{2}}\right)\right\} .
$$

Espera-se que, caso sejam satisfeitas as condições que foram assumidas, o valor de LoOP seja próximo de 1 se o ponto em questão for um outlier baseado em densidade, e 0 se for um 
ponto dentro de uma região densa. Segundo os autores, o algoritmo é robusto em relação ao valor de $\lambda \mathrm{e}$, nos experimentos realizados pelos mesmos, o valor de $\lambda$ foi 3 . Portanto, para os resultados deste documento, esse valor de $\lambda$ proposto pelos autores foi utilizado, variando apenas o parâmetro $k$ como nos algoritmos anteriores.

\subsubsection{INFLO}

O diferencial proposto por Jin et al. (2006) ao criar o algoritmo INFLO foi utilizar não apenas a relação de um ponto $p$ com seus vizinhos, mas também a influência que esse ponto $p$ tem para com outros pontos da base de dados. Segundo Jin et al. (2006), utilizar a inversa dos vizinhos mais próximos (Reverse Nearest Neighbors $(\mathrm{RNN})$ ) juntamente com os vizinhos mais próximos (Nearest Neighbors $(\underline{\mathrm{NN}})$ ) de um objeto $p$ torna o score produzido pelo algoritmo mais robusto e correto semanticamente comparado com outros métodos existentes.

Assim como nos algoritmos anteriores, definições formais do cálculo das distâncias entre um ponto e um conjunto de vizinhos mais próximos a ele foram necessárias. A Definição 10 utiliza a representação formal da distância de um ponto $p$ até seu $k$ vizinho mais próximo ( $k \_$distance $(p)$, ou $k_{\text {dist }}(p)$, vide Definição 5), para calcular o conjunto de vizinhos mais próximos à $p\left(N N_{k}(p)\right)$, em que $d(p, X)$ é o conjunto de distâncias entre um ponto $p$ e seus $k$ vizinhos mais próximos. Seguinte a esta, a Definição 11 apresenta o cálculo da densidade de um ponto $p$.

Definição 10. Seja $D$ uma base de dados, a distância para os $k$ vizinhos mais próximos ( $k$ nearest neighbors) de $p, N N_{k}(p)$ é um conjunto de objetos $X \in D$ tal que $d(p, X) \leq k_{\text {dist }}(p)$ : $N N_{k}(p)=\left\{X \in D \backslash\{p\} \mid d(p, X) \leq k_{\text {dist }}(p)\right\}$.

Definição 11. A densidade de p, escrita como den(p), é definida aqui como o inverso da distância entre o ponto p e seu $k$ vizinho mais próximo, i.e., den $(p)=1 / k_{\text {dist }}(p)$.

O diferencial deste algoritmo está na Definição 12, onde é formalizado o conceito de conjunto inverso de $k$ vizinhos mais próximos com relação a um ponto $p$. Esse conceito é utilizado juntamente com a Definição 10 de vizinhança mais próxima de um ponto $p$ para alcançar o score final produzido pelo algoritmo. Para possibilitar isso, o conceito de espaço de influência (Influence Space $(\underline{\mathrm{IS}})$ ) foi criado pelos autores, no qual é realizado uma união entre os elementos dos conjuntos $N N_{k}(p)$ e $R N N_{k}(p)$. Essa união foi escrita pelos autores como $I S_{k}(p)$.

Definição 12. O inverso dos $k$ vizinhos mais próximos $(\underline{R N N})$ é um conjunto definido pela seguinte relação inversa: $R N N_{k}(p)=\left\{q \mid q \in D, p \in N N_{k}(q)\right\}$.

Através de todas as definições acima, Jin et al. (2006) definiram o algoritmo INFLO conforme descrito na Equação 2.11). O score produzido pelo algoritmo é uma proporção da média das densidades dos objetos em $I S_{k}(p)$ (união entre $\underline{\mathrm{RNN}} \underline{\mathrm{KNN}}$ ) em relação à densidade local de $p$. O valor de INFLO será maior se a densidade de um ponto $p$ for menor que para aqueles 
objetos do seu espaço de influência (Jin et al., 2006).

$$
\begin{aligned}
& I N F L O_{k}(p)=\frac{\operatorname{den}_{a v g}\left(I S_{k}(p)\right)}{\operatorname{den}(p)} \\
& \text {,onde } \\
& \operatorname{den}_{\text {avg }}\left(I S_{k}(p)\right)=\frac{\sum_{o \in I S_{k}(p)} \operatorname{den}(o)}{\left|I S_{k}(p)\right|} .
\end{aligned}
$$

\subsection{6 $\mathrm{COF}$}

COF (Tang et al., 2002) é um algoritmo de detecção local de outliers que modifica a estimativa de densidade do LOF simplificado de acordo com a conectividade da vizinhança. Segundo Tang et al. (2002), este algoritmo se baseia na ideia de diferenciar baixa densidade de isolabilidade. Enquanto baixa densidade se refere ao fato de que a quantidade de objetos na vizinhança de um objeto é relativamente pequena, isolabilidade refere-se ao grau em que um objeto é "conectado" a outros objetos.

No algoritmo proposto, primeiramente para cada ponto $p$ na base de dados, um conjunto é formado contendo o ponto $p$ e os $k$ vizinhos mais próximos a $p\left(N_{k}(p)\right)$. Uma trilha é traçada a partir do ponto $p$ para todos os seus vizinhos mais próximos, conectando cada ponto do conjunto $N_{k}(p)$. Considerando que cada aresta corresponde à distância entre dois pontos, a distância média de conectividade do ponto $p\left(a c \_d i s t_{N_{k}(p)}\right)$ é definida como a média dos pesos das arestas em $N_{k}(p)$. A Equação 2.12) representa o score retornado pelo algoritmo COF Este score é definido pela razão da distância média de conectividade do ponto $p$ e das distâncias médias de conectividade dos $k$ vizinhos mais próximos de $p$ para seus próprios $k$ vizinhos mais próximos.

$$
C O F_{k}(p)=\frac{a c \_\operatorname{dist}_{N_{k}(p)}}{\sum_{o \in N_{k}(p)} a c \_\operatorname{dist}_{N_{k}(o)}} .
$$

\subsubsection{LDF}

A ideia proposta por Latecki et al.(2007) foi substituir a estimativa de densidade do LOFpor uma função de estimativa de densidade kernel gaussiana (Kernel Density Estimation (KDE)). Na formulação do KDE, a distância euclidiana é substituída pela distância de alcançabilidade local (vide Definição 7), a fim de se obter uma função de estimativa de densidade local (Local Density Estimation ([DDE) conforme a Equação 2.13). Na Equação 2.13), $k N N(p)$ indica a distância para o $k$ vizinho mais próximo de $p, d$ são as dimensões e $h$ a largura de banda do kernel.

$$
L D E(p)=\frac{1}{|k N N(p)|} \sum_{o \in k N N(p)} \frac{1}{(2 \pi)^{d / 2} h^{d}} e^{-\frac{1}{2} \frac{\text { reach_dist }(p, o)^{2}}{h^{2}}} .
$$

Após obter os valores de LDE para um ponto $p$ e seus $k$ vizinhos mais próximos, é possível determinar o score final de outlier para o ponto $p$ através da Equação (2.14). Nesta equação, $c$ 
$>0$ é uma constante bem pequena, necessária para a estabilidade da fórmula quando o $\mathrm{LDE}(p)$ alcança um valor próximo de 0 .

$$
L D F(p)=\frac{\frac{1}{k} \sum_{o \in k N N(p)} L D E(o)}{L D E(p)+\frac{c}{k} \sum_{o \in k N N(p)} L D E(o)} .
$$

\subsubsection{KDEOS}

Este algoritmo é uma recente variação do LOF, proposto por Schubert et al. (2014a). O KDEOS assume que a variabilidade da estimativa de densidade local é sensível à localidade. Assim como no $\mathrm{LDF}$ este modelo incorpora os conceitos de $\mathrm{KDE}$ no algoritmo LOF, Entretanto, ao contrário do realizado no $\mathrm{LDF}$ o estimador de densidade de kernel utilizado no KDEOS para estimar a densidade local é formalmente correto. Schubert et al. (2014a) indica que ao utilizar a distância de alcançabilidade local, o $\mathrm{LDE}$ perde as propriedades desejadas de uma estimativa correta de densidade de kernel: ao invés de ter uma área da integral de 1 para cada ponto, uma quantidade variável de peso é cortado do kernel de uma forma heurística.

Para calcular a KDE, Schubert et al. (2014a) sugerem a utilização de kernel Gaussiano ou kernel Epanechnikov. Após calcular os valores de $\mathrm{KDE}$, os scores relacionados a cada ponto $p$ são normalizados (utilizando a transformação z-score) em relação às estimativas associadas aos elementos do conjunto dos $k$ vizinhos mais próximos de $p$.

Os resultados obtidos utilizando a transformação z-score são tratados como normalmente distribuídos. Com isso, os autores utilizaram uma função de distribuição acumulada para mapear os scores obtidos no intervalo [0,1], e assim aplicar a transformação introduzida por Kriegel et al. (2012) conforme a Equação (2.15). Nesta equação, $\varphi$ é a taxa esperada de outliers e $p$ é o score a ser normalizado. O resultado final do KDEOS é o score obtido após a aplicação da normalização conforme a Equação 2.15).

$$
\operatorname{norm}(p, \varphi)=\frac{\varphi(1-p)}{\varphi+p} .
$$

\subsubsection{Fast $A B O D$}

Definido como uma versão mais rápida do algoritmo Angle-based Outlier Detection (ABOD), este método proposto por Kriegel et al. (2008) aborda uma visão diferente de detectar outliers. Kriegel et al. (2008) propõem usar não apenas a distância entre dois pontos, mas também as direções entre estes vetores de distância. Comparar os ângulos relacionados aos vetores de distâncias para outros pontos podem ajudar a discernir outliers e inliers.

Como explicação para definir a ideia utilizada pelos autores, a Figura 2.3 representa uma base de dados simples, representada bidimensionalmente. Ao selecionar um ponto dentro de um cluster, os ângulos entre diferentes vetores de distância deste ponto para com outros pontos da base possuirá uma grande variação. Esta variação tende a diminuir quando os pontos selecionados se situam na borda dos clusters (borderlines). E quando legítimos outliers surgirem, 
esta variância entre os ângulos tende a ser mínima.

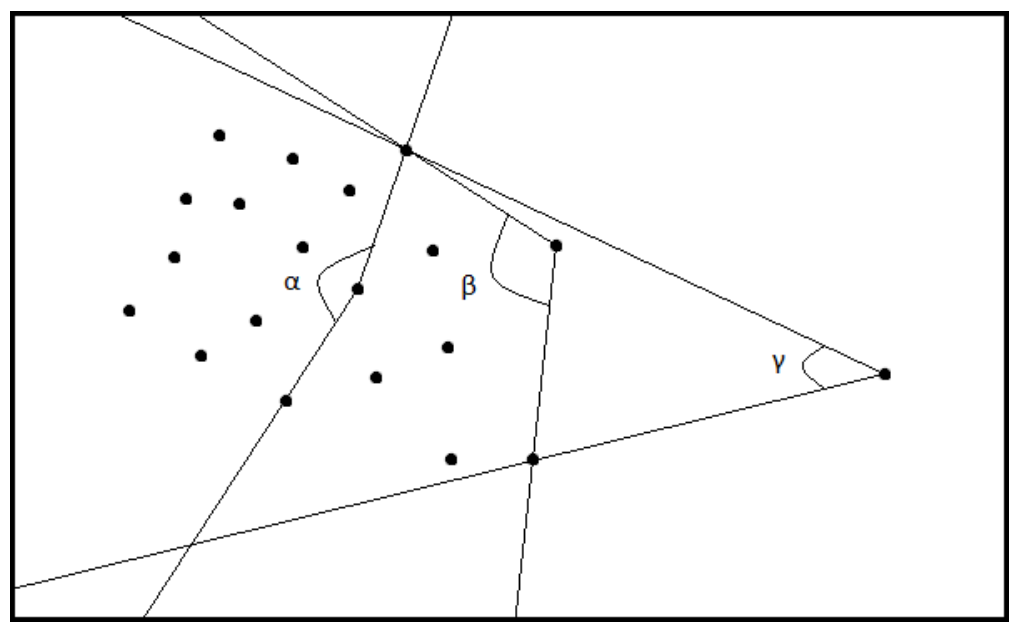

Figura 2.3: Intuição básica de detecção de outliers baseada em ângulo (Inspirado em (Kriegel et al., 2008))

O score final do algoritmo $\mathrm{ABOD}$ é dado pela variação dos ângulos entre diferentes vetores de distância deste ponto para com outros pontos da base ponderados pela distância dos pontos. Entretanto, realizar tal procedimento possui um custo computacional elevado $\left(O\left(N^{3}\right)\right)$, pois para cada ponto, todos os pares de pontos devem ser considerados. O algoritmo Fast ABOD é uma aproximação do algoritmo ABOD considerando em sua execução apenas os pares de pontos que estão dentro dos $k$ vizinhos mais próximos de um certo ponto selecionado. Com isso, o algoritmo resultante possui complexidade $O\left(k^{2} N^{2}\right)$.

\subsection{Avaliação de Algoritmos de Detecção de Outliers}

Os resultados finais de cada algoritmo de detecção de outliers, como aqueles apresentados nesse capítulo, são valores que buscam quantificar o quão anômala é cada instância da base.

Em classificação de dados, existem técnicas bem estabelecidas para avaliar algoritmos de classificação a partir de conhecimento a priori sobre a qual classe cada instância de uma base de dados pertence. Essa avaliação pode ser aplicada também em algoritmos da área de detecção de outliers se bases de dados rotuladas estiverem disponíveis, nas quais as instâncias da base que são de fato consideradas outliers são conhecidas a priori. Note que, no caso dos algoritmos de detecção não supervisionados, como aqueles discutidos neste capítulo, tais rótulos de classe binários (outliers e inliers), se disponíveis, não são utilizados na execução dos algoritmos, apenas na avaliação dos resultados desses frente a resultados esperados (em cenários em que esses são conhecidos).

Diversos trabalhos na área de detecção de outliers utilizam bases provindas da área de classificação (Gao and Tan, 2006; Wang and Davidson, 2009; Schubert et al., 2012), na qual os outliers são definidos de acordo com alguma semântica (e.g., uma classe rara). Nesse caso, os objetos anômalos nas bases são conhecidos previamente e, no caso dos algoritmos não supervisionados, são utilizados apenas para avaliação do desempenho desses algoritmos. Para facilitar 
esta avaliação pode-se transformar os scores produzidos por cada algoritmo em rankings, no qual o primeiro objeto do ranking é aquele objeto que possui o maior valor de score segundo a definição de outlier utilizada pelo algoritmo em questão, e assim por diante.

Uma técnica usual para comparar o desempenho de diferentes métodos, tanto na área de classificação quanto na área de detecção de outliers, é através da curva Receiver Operating Characteristic (ROC) (Han et al., 2011). Através desta técnica é possível comparar a taxa de verdadeiros positivos com a taxa de falsos positivos. Em detecção de anomalias, isto significa verificar a quantidade de verdadeiros outliers corretamente "classificados" (através dos rankings) com a quantidade de inliers "classificados" incorretamente como outliers pelo algoritmo. Um método perfeito de detecção de anomalias deve retornar primeiramente (primeiras posições do ranking) os objetos que são de fato outliers de acordo com o ground truth, seguido de todos os demais objetos da base (Kriegel et al., 2009a).

Pode-se afirmar que a curva $\mathrm{ROC}$ é uma representação bidimensional (True Positive Rate (TPR) e False Positive Rate ( $(\overline{\mathrm{FPR}})$ ) de avaliação. Com o objetivo de sumarizar os desempenhos de algoritmos em um único valor escalar, o método de calcular a área abaixo da curva ROC (AUC) tem sido usualmente utilizado na comparação/avaliação de técnicas de detecção de outliers (Kriegel et al., 2011; Zimek et al., 2012; Schubert et al., 2012; Zimek et al., 2013a). $\mathrm{O}$ valor de $\mathrm{AUC}$ igual a 1 significa uma separação perfeita entre outliers e inliers. Já o valor de AUC igual a 0.5 indica o aumento simultâneo entre a taxa de verdadeiros positivos e falsos positivos, o que é o resultado esperado para uma rotulação meramente aleatória. Por fim, uma área abaixo da curva $\mathrm{ROC}$ com valor 0 indica que todos os inliers foram retornados primeiros do que os outliers no ranking do algoritmo.

Todos os algoritmos apresentados neste capítulo executam através de um dado parâmetro $k$, o qual altera a quantidade de vizinhos mais próximos (e, portanto, a abrangência ou escopo da vizinhança) de um dado ponto $p$ a ser considerado. Na prática, não é trivial selecionar o melhor valor de $k$ para as técnicas não supervisionadas. Entretanto, em experimentos controlados em que se utilizam bases de dados rotuladas, o valor de AUC é uma ótima opção para determinar qual $k$ obteve o melhor resultado para um dado algoritmo. Mais do que isso, esse valor de área abaixo da curva $\mathrm{ROC}$ permite identificar qual método com o seu melhor valor de $k$ obteve o melhor desempenho dentre outros valores de $k$ e algoritmos em uma determinada base de dados onde se conhece previamente quais são os outliers.

Uma outra medida simples para calcular a performance dos algoritmos é a precision at $n$ $(P @ n)$. Esta medida é definida pela proporção de outliers corretos entre os $n$ primeiros no ranking (Craswell, 2009a). Considerando uma base de dados $D B,|D B|=N$, composta por outliers $O \subset D B$ e inliers $I \subseteq D B$ (em que $D B=O \cup I$ ), a $P @ n$ pode ser formalizada como descrito pela Equação 2.16.

$$
\underline{P @ n}=\frac{|\{o \in O \mid \operatorname{rank}(o) \leq n\}|}{n} .
$$

Considerando as medidas de avaliação $\mathrm{AUC}$ e $P @ n$, é importante destacar que a primeira considera o ranking total das instâncias enquanto a segunda verifica a precisão de detecção dos 
outliers nas $n$ primeiras posições. Ambas as medidas geram resultados complementares e que revelam diferentes aspectos da qualidade de detecção, visto que altos valores de AUC indicam que os outliers estão relativamente acima dos inliers nas posições do ranking de detecção, entretanto eles não estão necessariamente entre as primeiras posições. Portanto, saber os valores destas duas medidas de avaliação permitem analisar o ranking das instâncias gerado por um algoritmo de detecção e a precisão (presença de outliers) no topo deste ranking.

Neste trabalho nós assumimos que os rankings dos outliers são únicos, porém caso haja dois ou mais objetos compartilhando o mesmo score de outlier, consideramos que os empates são resolvidos arbitrariamente, mas consistentemente, utilizando a média dos rankings para todas as instâncias empatadas.

Tendo em vista o desequilíbrio existente entre a quantidade de outliers e inliers presentes nas bases de dados, no qual esperamos uma quantidade bem maior de inliers, a medida $P @ n$ tem dificuldade nesses casos. Por exemplo, para uma base de dados com 10 outliers e 1 milhão de inliers, um algoritmo que atribui os verdadeiros outliers para os rankings 11-20 (bastante elevados) terá, no entanto, uma P@10 de 0, mas uma P@20 de 0.5.

Ao utilizar $P @ n$ para avaliar o desempenho geral de um método de detecção de outliers, ainda é incerto como escolher de uma maneira justa o valor do parâmetro $n$. Uma escolha comum na literatura, e portanto adotada neste trabalho, é fazer o parâmetro $n$ como o número de verdadeiros outliers na base, $n=|O|$ (também conhecida como R-Precision (Craswell, $2009 \mathrm{~b})$ ). Sempre que o número de outliers é baixo em relação à base de dados, os valores de $P @ n$ que são obtidos podem ser enganosamente baixos e não muito informativos. Por outro lado, quando a quantidade de outliers é alta em relação à base de dados, valores enganosamente altos de $P @ n$ são obtidos simplesmente devido ao número relativamente pequeno de inliers disponíveis (vide exemplos no final deste capítulo). Com o intuito de resolver este problema, é adotado aqui um ajuste por chance na métrica $P @ n$.

Ajuste por chance é um procedimento de princípios que foi discutido por Hubert and Arabie (1985) para validação de clusters, mas que pode ser aplicado em quaisquer índices como apresentado na Equação (2.17).

$$
\text { ÍndiceAjustado }=\frac{\text { Índice }- \text { ÍndiceEsperado }}{\text { ÍndiceMáximo }- \text { ÍndiceEsperado }} .
$$

O valor máximo possível de $P @ n$ é $\frac{|O|}{n}$ se $n>|O|$, e 1 caso contrário. O valor esperado sob a hipótese de um ranking completamente aleatório dos outliers é $\frac{|O|}{N}$, o qual não depende de $n$. Assumindo que $n \leq|O|$, obtemos a Equação (2.18). Para valores grandes de $n$, obtemos a Equação 2.19).

$$
\begin{aligned}
& \text { Adjusted } \overline{\mathrm{P} @ \mathrm{n}}=\frac{\overline{\mathrm{P} @ \mathrm{n}}-\frac{|O|}{N}}{1-\frac{|O|}{N}} . \\
& \text { Adjusted } \mathrm{P@n}=\frac{\mid \mathrm{P} @ \mathrm{n}-\frac{|O|}{N}}{\frac{|O|}{n}-\frac{|O|}{N}} .
\end{aligned}
$$


Além da medida de avaliação $R$-Precision $(P @|O|)$, que representa uma escolha particular em que $n=|O|$, tem sido dada também atenção na literatura às medidas que agregam desempenho ao longo de uma ampla gama de possíveis escolhas de $n$. Uma dessas medidas, popular em contextos de recuperação de informação, é a precisão média (AP) (Zhang and Zhang, 2009), representada pela Equação (2.20).

$$
\underline{\mathrm{AP}}=\frac{1}{|O|} \sum_{o \in O} P @ \operatorname{rank}(o) .
$$

Ao invés de avaliar apenas em um único valor de $n$, os valores de $P @ n$ são calculados através da média dos rankings de todos os outliers presentes na base de dados (de acordo com o ground truth). Existem outras variantes, tais como uma amostra dos rankings em intervalos fixos, no entanto a avaliação nos rankings positivos (outliers) parece ser popular e bem adequada para conjuntos de dados desbalanceados.

A precisão média pode ser ajustada da mesma forma como realizado na medida $P @ n$. Um ranking perfeito produz o valor máximo de 1, e o valor esperado de um ranking aleatório é $\frac{|O|}{N}$. Por tudo isso apresentado, a medida de precisão média ajustada é representada pela Equação (2.21).

$$
\text { Adjusted } \mathrm{AP}=\frac{\mid \mathrm{AP}-\frac{|O|}{N}}{1-\frac{|O|}{N}} .
$$

Considere os rankings presentes nas Tabelas 2.1 e 2.2 como resultados de um algoritmo de detecção de outliers em uma base de dados com 10 instâncias , junto aos resultados das métricas de avaliação. Na Tabela 2.1 existem 2 outliers e 8 inliers, o que sugere um cenário real em que a quantidade de inliers é bem superior à quantidade de outliers. Os dois outliers nesta tabela não estão nas duas primeiras posições do ranking, porém eles aparecem logo em seguida nas posições 3 e 4 . Ao aplicar as medidas de avaliação neste ranking (utilizando $n=|O|$ ), conseguimos verificar a complementariedade entre as métricas $P @ n$ e AUC, em que apesar de ambos os outliers estarem relativamente bem posicionados $(\mathrm{AUC}=0.75)$, nenhum dos outliers

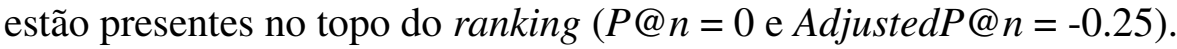

A Tabela 2.2 é um exemplo de como os valores das métricas $P @ n$ e AP podem ser enganosamente altos quando existem muitos outliers na base de dados, e como o ajuste proposto informa valores mais realísticos considerando o ranking. Este exemplo é composto por 7 outliers e 3 inliers. Considerando que todos os 3 inliers estão presentes nas três primeiras posições do ranking conforme a Tabela 2.2, percebemos que claramente este ranking é o pior caso possível (inliers classificados acima dos outliers). Contudo, as medida $P @$ e $\mathrm{AP}$ geram os resultados 0.5714 e 0.5304 respectivamente, que são obtidos pelo simples fato da base de dados possuir muitos outliers. Aplicando o ajuste mencionado anteriormente, os resultados foram -0.4287 e -0.5653 para as métricas Adjusted $\overline{P @ n}$ e Adjusted $\mathrm{AP}$ respectivamente, tornando os valores mais realísticos considerando o ranking.

Essas medidas (Adjusted $P @ n$ e Adjusted $(\mathrm{AP})$ foram originalmente propostas em Campos et al. (2015) (submetido) com a participação do autor desta dissertação. Por fim, todas estas 
métricas apresentadas serão utilizadas para avaliar comparativamente o desempenho dos algoritmos de detecção de anomalias. Os resultados são apresentados e discutidos no Capítulo 4 deste documento.

Tabela 2.1: Ranking retornado de um algoritmo de detecção de outliers e os resultados das métricas de avaliação. Complementariedade das métricas AUC e $P @ n$ (utilizando $n=|O|$ ).

\begin{tabular}{|c|c|}
\hline Ranking & Label \\
\hline 1 & $\mathrm{I}$ \\
\hline 2 & $\mathrm{I}$ \\
\hline 3 & $\mathrm{O}$ \\
\hline 4 & $\mathrm{O}$ \\
\hline 5 & $\mathrm{I}$ \\
\hline 6 & $\mathrm{I}$ \\
\hline 7 & $\mathrm{I}$ \\
\hline 8 & $\mathrm{I}$ \\
\hline 9 & $\mathrm{I}$ \\
\hline 10 & $\mathrm{I}$ \\
\hline
\end{tabular}

\begin{tabular}{|c|c|c|c|c|}
\hline $\mathbf{A U C}$ & $\overline{\boldsymbol{P} @ \boldsymbol{n}}$ & $\mathbf{A P}$ & Adjusted $\overline{\mathbf{P} @ \mathbf{n}}$ & Adjusted $\mathbf{A P}$ \\
\hline 0.75 & 0 & 0.4166 & -0.25 & 0.2708 \\
\hline
\end{tabular}

Tabela 2.2: Ranking retornado de um algoritmo de detecção de outliers e os resultados das métricas de avaliação. Resultados enganosamente altos para as medidas $P @ n$ e $\mathrm{AP}$ (utilizando $n=|O|)$.

\begin{tabular}{|c|c|}
\hline Ranking & Label \\
\hline 1 & $\mathrm{I}$ \\
\hline 2 & $\mathrm{I}$ \\
\hline 3 & $\mathrm{I}$ \\
\hline 4 & $\mathrm{O}$ \\
\hline 5 & $\mathrm{O}$ \\
\hline 6 & $\mathrm{O}$ \\
\hline 7 & $\mathrm{O}$ \\
\hline 8 & $\mathrm{O}$ \\
\hline 9 & $\mathrm{O}$ \\
\hline 10 & $\mathrm{O}$ \\
\hline
\end{tabular}

\begin{tabular}{|c|c|c|c|c|}
\hline $\mathbf{A U C}$ & $\overline{P @ n}$ & $\mathbf{A P}$ & Adjusted $\overline{\mathbf{P} @ n}$ & Adjusted $\mathbf{A P}$ \\
\hline 0 & 0.5714 & 0.5304 & -0.4287 & -0.5653 \\
\hline
\end{tabular}




\section{Benchmarks de Bases de Dados para Detecção de Outliers}

\subsection{Definição e Motivação}

Uma das dificuldades para o estabelecimento de bases de dados benchmark é a subjetividade do próprio conceito de outlier. Diferentes intuições sobre este conceito possivelmente demandam que benchmarks de naturezas variadas sejam considerados nos processos de avaliação. Por exemplo, alguns outliers podem se caracterizar como tal condicionalmente a determinados fatores, e.g., uma dada temperatura que pode ser extremamente incomum numa dada estação do ano, mas muito comum em outra estação. Tais tipos de outliers são denominados condicionais (Song et al., 2007; Wang and Davidson, 2009). Outros outliers podem se destacar como observações excepcionais apenas quando parte dos atributos que descrevem as observações são considerados. Algoritmos que buscam por tal tipo de outlier são denominados algoritmos de detecção em subespaços (Kriegel et al., 2009b; Zimek et al., 2012). Alguns outliers podem ainda se diferenciar por representarem observações com comportamento temporal ou sequencial extraordinários (Chandola et al., 2012).

Em alguns trabalhos na literatura envolvendo avaliação de algoritmos de detecção (e.g. (Zhang et al., 2009; Keller et al., 2012)) utilizam-se versões modificadas de bases de dados de classificação (e.g. bases do repositório UCI (Frank and Asuncion, 2010)), nas quais uma das classes é subamostrada para assegurar que as respectivas observações são pouco frequentes com relação às demais classes. Esta classe subamostrada é considerada então como a "classe outlier" simplesmente porque agora ela é "rara". Entretanto, características (estatísticas, espaciais, estruturais, etc) das diferentes classes, conforme descritas pelos atributos da base de dados, não são consideradas neste tipo de rotulação arbitrária. Mais além, se a classe outlier 
é escolhida sem que se use um critério apropriado, é provável que não haja qualquer compatibilidade entre a semântica dos dados e as rotulações das observações/classes como outliers e inliers. Por exemplo, é mais natural que, em uma base contendo registros de transações financeiras, as transações fraudulentas sejam aquelas consideradas outliers, não o oposto. Como um outro exemplo, também é mais natural esperar que, dentre uma coleção diversificada de vinhos, alguns poucos vinhos sejam excepcionalmente ruins ou excepcionalmente bons e que estes sejam considerados os outliers. Neste sentido, também é geralmente esperado que haja um desbalanceamento natural entre as proporções de outliers e inliers, que idealmente não precisaria ser artificialmente imposto por um procedimento de subamostragem. Por estas razões, o tipo de procedimento descrito acima, caso realizado de forma simplista e sem os devidos cuidados, pode resultar em benchmarks que podem ser bons ou ruins puramente ao acaso (em que "bom" e "ruim" podem refletir características como "fácil" ou "difícil", semanticamente compatível ou não, etc).

Um ponto interessante ao processar bases de dados de interesse é o grau de dificuldade com que as observações consideradas outliers são detectadas. Dada uma base de dados é possível modificar este grau, por exemplo, eliminando parte dos atributos que descrevem as observações. Para constituir um bom benchmark, é ideal que seja difícil encontrar os outliers no espaço de atributos em que estão descritos, mas não impossível. Intuitivamente, um bom benchmark parece ser tal que, em sua maioria, cada outlier possa ser encontrado por ao menos um algoritmo existente, porém uma minoria possa ser encontrada por muitos algoritmos. Utilizar os próprios algoritmos para este tipo de análise, entretanto, requer cautela, especialmente caso se pretenda avaliar não apenas novos algoritmos a serem futuramente desenvolvidos, mas também os próprios algoritmos já existentes utilizando a coleção de bases a ser desenvolvida.

\subsection{Propostas na Literatura}

De acordo com as considerações apresentadas na Seção 3.1, para criação de uma boa coleção benchmark para avaliação de algoritmos de detecção não supervisionada de outliers, foi proposto por Emmott et al. (2013) uma metodologia para transformar bases de dados existentes nas áreas de classificação e regressão em bases para detecção de anomalias. Para isso, os autores definiram quatro requisitos a serem respeitados. Esses requisitos são importantes e refletem a necessidade e a dificuldade da construção de benchmarks para detecção de anomalias.

Requisito 1: Pontos normais (não anômalos) devem ser descritos por um processo de geração de dados baseados no mundo real. Gerar conjuntos de dados através de distribuições de probabilidade arrisca não capturar processos do mundo real.

Requisito 2: Pontos anômalos devem também ser descritos por um processo de geração de dados baseados no mundo real, que seja semanticamente distinto do processo de geração de pontos normais. Os outliers não devem ser apenas pontos na cauda de uma distribuição normal. 
Requisito 3: Várias bases de dados benchmark são necessárias. Caso seja utilizada apenas uma quantidade muito pequena de bases de dados há o risco dos algoritmos a serem desenvolvidos funcionarem apenas nestas bases.

Requisito 4: Um benchmark de bases de dados deve ser caracterizado por problemas bem definidos e com "dimensões" (aspectos) significativas que podem variar sistematicamente. Um objetivo importante em construir uma coleção benchmark de bases de dados é compreender melhor os pontos fortes e pontos fracos de vários algoritmos de classificação/agrupamento ou de detecção de outliers. Para tal, é preciso identificar os aspectos ao longo dos quais os problemas de detecção de anomalias podem variar e, com isso, gerar uma coleção benchmark de bases de dados que varia esses aspectos de forma controlada.

Nesse trabalho recentemente publicado, Emmott et al. (2013) pré-processam diversas bases de dados recolhidas do repositório UCI (Frank and Asuncion, 2010) para construir uma coleção benchmark para detecção de outliers. Porém, ao transformar as bases de classificação binária coletadas para o contexto de detecção de anomalias, Emmott et al. (2013) escolheram uma classe "normal" e uma classe "anômala". Essa atribuição arbitrária vai contra questões ditas anteriormente neste mesmo capítulo, em que ao realizar essa atribuição perde-se, em muitos casos, a semântica do conceito de outlier. Outro ponto questionável utilizado pelos autores foi realizar indiscriminadamente uma normalização de todos os atributos no intervalo entre -1 e 1 para todas as bases de dados. Normalizar os dados pode ser em muitos casos importante se houver uma dominância artificial de alguns atributos meramente por variações grandes de escala. Esta abordagem, entretanto, pode não ser necessariamente apropriada já que, ao normalizar todos os atributos, alguns outliers que poderiam ser detectados no espaço original dos dados podem ser mascarados em meio à normalização.

Emmott et al.(2013) citam a importância de desenvolver métodos para controlar a proporção de atributos relevantes e irrelevantes. Tais métodos ajudam o desenvolvimento de melhores técnicas de seleção de atributos em algoritmos de detecção de anomalias.

Outro aspecto abordado por Emmott et al. (2013) é um valor que demonstra o grau de dificuldade para detectar outliers em uma base de dados. Esse valor é denominado de dificuldade de ponto e foi calculado por Emmott et al. (2013) através da aplicação de um classificador de regressão logística baseado em kernel em cada uma das bases selecionadas. Utilizar as probabilidades retornadas por uma regressão logística com kernel permite quantificar o quão provável é um ponto (instância) rotulado como outlier ser classificado como tal, o que pode ser interpretado como o grau de dificuldade para discriminar esta instância dos inliers (detectá-la outlier). Contudo, para executar a regressão logística com kernel é necessário configurar parâmetros. Estimar estes parâmetros é uma tarefa não trivial e os autores não explicaram como abordaram este problema, muito menos disponibilizaram publicamente as bases resultantes dos procedimentos de pré-processamento por eles descritos.

Várias bases utilizadas por Emmott et al. (2013) serão também consideradas no presente projeto (vide Capítulo 4). Aqueles autores selecionaram 19 bases do repositório UCI agru- 
pados em três diferentes categorias naturais: classificação binária, classificação multiclasse e regressão. As bases estão representadas na Tabela 3.1. Contudo, nem todas as bases utilizadas possuem uma semântica clara do que realmente são outliers. Por isso, no presente projeto, diversas outras bases serão utilizadas, as quais possuem uma diferenciação semântica entre inlier e outlier. Bases comumente utilizadas na literatura nesta área de detecção não supervisionada de anomalias também serão abordadas. Os resultados estão discutidos minuciosamente no Capítulo 4, e a proposta de criação de uma coleção benchmark de bases de dados para detecção de outliers é abordada na subseção seguinte.

Tabela 3.1: Bases de dados utilizadas por Emmott et al. (2013).

\begin{tabular}{|c|c|}
\hline Bases de Dados & Tarefa \\
\hline MAGIC Gamma Telescope & classificação binária \\
\hline Mini-BooNE Particle Identification & classificação binária \\
\hline Sking Segmentation & classificação binária \\
\hline Spam-base & classificação binária \\
\hline Steel Plates Faults & classificação multiclasse \\
\hline Gas Sensor Array Drift & classificação multiclasse \\
\hline Image Segmentation & classificação multiclasse \\
\hline Landsat Satelite & classificação multiclasse \\
\hline Letter Recognition of Hand-written Digits & classificação multiclasse \\
\hline Page Blocks & classificação multiclasse \\
\hline Shuttle & classificação multiclasse \\
\hline Waveform & classificação multiclasse \\
\hline Yeast & classificação multiclasse \\
\hline Abalone & regressão \\
\hline Communities and Crime & regressão \\
\hline Concrete Compressive Strenght & regressão \\
\hline Wine & regressão \\
\hline Year Prediction & regressão \\
\hline
\end{tabular}

\subsection{Propostas deste Projeto}

Assim como consta nos objetivos deste projeto, a criação de uma coleção benchmark de bases de dados para detecção de outliers deve ser cuidadosamente viabilizada. Com o trabalho de Emmott et al.(2013) nesta área e as críticas citadas anteriormente, é possível a idealização de uma metodologia de desenvolvimento para criação de benchmarks voltados para algoritmos de detecção não supervisionada de outliers. A metodologia utilizada é composta pelos seguintes passos:

1. Análise e pré-seleção de bases de dados. 
2. Pré-processamento.

3. Aplicação de algoritmos representativos de detecção de outliers nas bases e geração de estatísticas de interesse.

4. Aplicação de técnicas de seleção de atributos em um período posterior à análise dos resultados

As subseções a seguir descrevem resumidamente cada uma destas etapas, relevando o objetivo em cada uma delas juntamente com o objetivo final do projeto.

\subsubsection{Análise e Pré-Seleção de Bases de Dados}

Diversos trabalhos na literatura sobre detecção de outliers utilizam bases de dados de classificação que não possuem compatibilidade semântica entre os rótulos originais das classes e as rotulações das instâncias como outliers e inliers. Contudo, assim como discutido no início deste capítulo, é importante que haja esta compatibilidade. Podem até existir casos em que uma base de dados possua mais outliers do que inliers, se os mesmos possuírem uma semântica clara de outlier (por exemplo, uma base de dados com mais registros de pacientes doentes que saudáveis), mas isso deve ser apropriadamente considerado pois viola a premissa que outliers são raros ou menos comuns que inliers.

As bases que foram coletadas estão analisadas e avaliadas no Capítulo 4 . Foram consideradas bases que possuem uma semântica clara entre inliers e outliers e bases que são comumente utilizadas na literatura de detecção de anomalias. O importante desta composição de bases de dados é não obter apenas bases que possuem instâncias raras, mas sim bases que possuem a semântica de outlier. O objetivo final desta etapa é obter uma coleção de bases que diversificam no quesito da subjetividade deste conceito (outlier). A avaliação da presença de semântica entre outliers e inliers foi definida após longas análises de cada base de dados em respeito aos conceitos de excepcionalidade e raridade dos eventos.

\subsubsection{Pré-Processamento}

\subsubsection{Bases Normalizadas e Não Normalizadas}

Ao pré-processar as bases coletadas na etapa anterior, é importante a manutenção de duas versões de uma mesma base: normalizada e não normalizada. Em cada caso pode ser possível, ou mais fácil, identificar diferentes outliers, ou seja, quando os dados estão naturalmente distribuídos, pode ocorrer que uma instância anômala seja mais facilmente detectada do que quando aplicado um processo de normalização nos atributos. Por isso, serão mantidas estas duas versões de cada base de dados, considerando os atributos em escala original e normalizados. 


\subsubsection{Atributos Faltantes e Atributos Categóricos}

Muitas bases não possuem todos os valores dos atributos para todas as instâncias. Existe uma área de pesquisa que busca averiguar qual seria a melhor forma de tratar tais valores (geralmente determinados por “?”). Na metodologia proposta foi determinada a remoção de atributos para os quais grande parte das instâncias possuem valores faltantes (mais de 10\% da base de dados); caso contrário, as instâncias com valores faltantes são removidas e os atributos mantidos.

Outro ponto importante é o tratamento dado a atributos categóricos. Muitos métodos de detecção de outliers não conseguem trabalhar com atributos categóricos e tais atributos aparecem em algumas bases de dados. Para contornar este problema, as bases que possuem atributos categóricos foram divididas em três versões: (a) transformando em atributos numéricos através da codificação 1-de-n, (b) transformando em atributos numéricos através da técnica Inverse Document Frequency (IDF) e (c) removendo os atributos categóricos. Mapear os atributos categóricos em numéricos utilizando a codificação 1-de-n consiste em simplesmente substituir cada atributo categórico por $n$ atributos binários, em que 1 representa a presença do valor categórico correspondente. Utilizar a técnica 【DF para transformar atributos categóricos em numéricos já foi utilizado anteriormente no contexto de detecção de anomalias por Lazarevic and Kumar (2005). Esta técnica utiliza a fórmula apresentada pela Equação (3.1), em que $N$ é o número total de instâncias da base de dados e $f_{t}$ é a frequência (número de ocorrências) do valor $t$ do atributo. Remover as instâncias é uma forma mais simples de tratar os atributos e também será averiguada nos resultados como uma das alternativas de tratar atributos categóricos.

$$
I D F=\ln \frac{N}{f_{t}}
$$

\subsubsection{Instâncias Duplicadas}

Vários algoritmos de detecção de anomalias possuem dificuldade ao detectar outliers quando a base de dados possui instâncias duplicadas. Isso é devido ao fato de alguns algoritmos utilizarem conceitos de densidade local das instâncias que, na presença de duplicatas, pode gerar valores infinitos. Entretanto, remover tais instâncias pode ser prejudicial por alterar a natureza dos dados presente na base. Com isso em mente, no decorrer das execuções deste projeto, foram mantidas as bases de dados sem a remoção de duplicatas e com a remoção de duplicatas. Ambas as versões estão presentes no benchmark de bases de dados para detecção de outliers criado, aumentando ainda mais a quantidade de bases utilizadas no projeto e, consequentemente, a relevância deste projeto para a área de detecção de anomalias.

\subsubsection{Subamostragem}

Realizar uma subamostragem dos dados é um procedimento comum em detecção de anomalias. A maioria das bases de dados utilizadas nesta área para avaliar algoritmos são coletadas da área de classificação e, portanto, a presença de várias instâncias na classe considerada outlier pode ser artificialmente alta, o que não condiz com a intuição por trás do conceito de outlier. 
O procedimento mais comum utilizado é subamostrar a classe outlier, deixando apenas poucas instâncias remanescentes desta classe e uma vasta quantidade de instâncias da classe inlier (o que simularia uma situação mais realística destes conceitos). Portanto, todas as bases de dados coletadas que possuem semântica entre outliers e inliers terão a classe outlier subamostrada para $20 \%, 10 \%, 5 \%$ e $2 \%$ (quando possível). Para cada percentual escolhido, a subamostragem é realizada 10 vezes com o intuito de minimizar o efeito de aleatoriedade. Tal procedimento implica um crescimento significativo da quantidade de bases de dados disponíveis no benchmark.

\subsubsection{Aplicação de Algoritmos Representativos de Detecção de Outli- ers nas Bases e Geração de Estatísticas de Interesse}

Após obter uma coleção de bases de dados, o procedimento utilizado foi aplicar um conjunto representativo de algoritmos de detecção não supervisionada de outliers com o intuito de analisar a dificuldade encontrada ao detectar outliers em cada base de dados. Após aplicar tal conjunto de algoritmos, foi possível avaliar comparativamente os algoritmos de detecção não supervisionada de outliers em uma coleção grande e diversa de bases de dados, o que nunca foi feito anteriormente. Conforme mencionado anteriormente, uma boa coleção benchmark parece ser tal que em sua maioria cada outlier possa ser encontrado por ao menos um algoritmo existente, porém uma minoria possa ser encontrada por muitos. Com isso, torna-se interessante a utilização de representantes de categorias de algoritmos propostas até o presente momento para verificar, através das medidas de avaliação mencionadas anteriormente neste documento, a acurácia de cada algoritmo ao detectar outliers. É importante também manter em uma base de dados outliers que não sejam detectados por nenhum algoritmo atualmente, pois isso traz um nível de dificuldade e impõe desafios para o desenvolvimento de novas abordagens de detecção.

Caso apenas uma minoria dos outliers presentes em uma certa base sejam descobertos por muitos algoritmos, considera-se que tal base impõe algum nível de dificuldade. Determinar este grau de dificuldade dos outliers está intimamente correlacionado com o seu comportamento perante as outras instâncias da base. Caso um outlier possua características muito distintas do restante da base de dados, ele tende a ser fácil de detectar. Entretanto se um outlier está próximo a um grupo de dados (chamado de borderline), o grau de dificuldade para detectá-lo aumenta, até chegar em um ponto onde o outlier está completamente em meio a um cluster, tornando impossível detectá-lo de forma não supervisionada.

A abordagem proposta por Emmott et al. (2013) é utilizar a Regressão Logística com Kernel (Kernel Logistic Regression (KLR) ) que atribui para cada instância uma probabilidade da mesma pertencer a cada uma das classes (em nosso caso: outlier e inlier). O problema maior encontrado ao utilizar esta abordagem é a parametrização necessária para trabalhar com a Regressão Logística com Kernel. Emmott et al. (2013) não explicam quais os valores dos parâmetros utilizados em seus experimentos, o que dificulta a utilização de tal técnica.

Neste trabalho, avançamos em relação à dificuldade de detectar outliers nas bases de dados coletadas utilizando mapas de calor (heatmaps) para determinar a dificuldade de cada método em detectar os outliers de uma base de dados. Com esses mapas de calor disponíveis, foi pos- 
sível analisar estatisticamente a dificuldade e a diversidade na detecção das anomalias, contribuindo para a análise da dificuldade de ponto para cada base de dados. Os avanços e resultados desta parte estão disponíveis no Capítulo 4

\subsubsection{Seleção de Atributos}

Nem todos os outliers são detectáveis no espaço completo de atributos. Conseguir determinar atributos relevantes nos quais outliers estão mais "visíveis" é um grande desafio atualmente na área de detecção de anomalias. Para fins de pré-processamento de bases com rotulação externa disponível, uma opção investigada neste trabalho é a utilização de árvores de decisão, para classificar as instâncias que são outliers e com isso determinar quais atributos são relevantes para o classificador discriminar tais outliers dos inliers. Este problema possui uma relação muito grande com o problema de classificação desbalanceada (mais inliers do que outliers), o que possibilita fazer uso de uma matriz de custo para "equilibrar" as classes. Tal matriz de custo atribui um peso maior ao classificar incorretamente um outlier, considerando que outliers sejam mais raros na base de dados. Com isso, classificar incorretamente todos os outliers deve possuir um peso idêntico ao classificar incorretamente todos os inliers. As árvores de decisão que buscam minimizar o custo embutido nessa matriz tendem a criar caminhos para classificar outliers e inliers. Estes caminhos são compostos por conjuntos de atributos, os quais permitem diferenciar as duas classes (normal e anômala), sendo possível assim definir outliers em uma parte dos atributos.

Outra abordagem alternativa é utilizar uma árvore de decisão sem poda. Tal árvore seria capaz de classificar corretamente todas as instâncias da base de dados, diferenciando outliers de inliers. Com isso, seria possível determinar quais atributos são relevantes para discriminar uma classe da outra.

Estas técnicas de seleção de atributos foram aplicadas numa etapa posterior às coletas de estatísticas de interesse. Apenas bases de dados em que os algoritmos de detecção tiveram desempenhos inferiores à um limiar tiveram estas técnicas aplicadas e seus resultados analisados. 


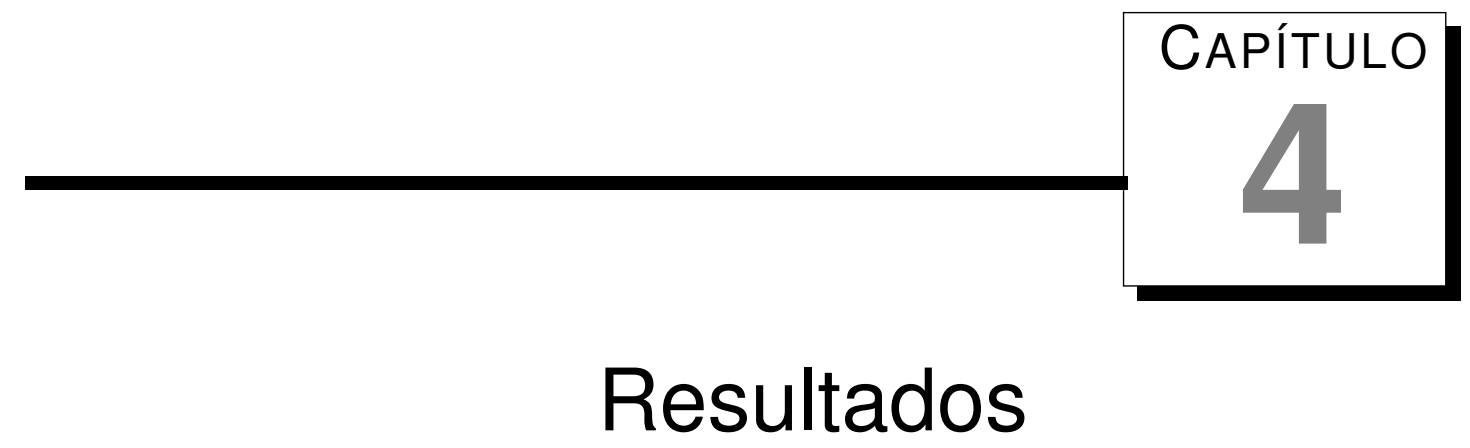

Neste capítulo serão apresentados os resultados obtidos ao aplicar técnicas de detecção não supervisionada de outliers em algumas bases de dados disponíveis publicamente. Para isso, este capítulo foi dividido em duas seções. A Seção 4.1 explica quais são as bases coletadas até o momento, o porquê destas bases para detecção de outliers e o pré-processamento realizado nelas. A Seção 4.2 mostra os resultados (considerando as bases de dados e os algoritmos) referentes à aplicação de algoritmos de detecção não supervisionada de outliers nas bases apresentadas, e o pós-processamento (seleção de atributos) realizado em algumas das bases de dados.

\subsection{Bases de Dados}

Todo o pré-processamento realizado nas bases de dados coletadas foi executado conforme explicado na Seção 3.3.2. O benchmark de bases de dados para detecção de outliers está disponível, juntamente com os resultados da seção seguinte, em nosso repositório on-line ${ }^{1}$

\subsubsection{Bases de Dados com Semântica Clara entre Outliers e Inliers}

A lista a seguir é uma compilação das bases de dados que possuem semântica e foram coletadas do repositório UCI (Frank and Asuncion, 2010). Para a composição do benchmark, todas as bases foram representadas por seus dados completos, unindo os conjuntos de treinamento e teste quando estes são representados separadamente.

- Annthyroid: Esta base possui registros clínicos referentes ao hipotireoidismo. Existem três classes: normal, hiperfunção e funcionamento subnormal. As classes relacionadas a disfunções da tireóide foram definidas aqui como outliers. Caso o paciente seja normal

${ }^{1}$ http://lapad-web.icmc.usp.br/repositories/outlier-evaluation/ 
(não possui hipotireoidismo), ele pertence aos inliers. Esta base possui 21 atributos numéricos e 7200 instâncias, divididas em 534 outliers (7.42\%) e 6666 inliers (92.58\%). Importante ressaltar que esta base possui 71 instâncias que são duplicatas e todas pertencem aos inliers.

- Pima: Esta base contém dados de pacientes que possuem ou não diabetes. A divisão em normal e anômalo nesta base é trivial, no qual pacientes com diabetes foram considerados outliers e pacientes que não possuem diabetes foram considerados inliers. Esta base possui 8 atributos numéricos e 768 instâncias, divididas em 268 outliers (34.9\%) e 500 inliers $(65.1 \%)$.

- Arrhythmia: Esta base classifica um paciente como normal ou contendo algum tipo de arritmia cardíaca. No total existem 14 tipos de arritmia diferentes e 1 tipo que reúne todos os demais tipos diferentes dos 14 anteriores. Contudo, 3 tipos de arritmia não possuem nenhuma instância representativa na base. Neste caso, considera-se aqui que a classe inlier possui as pessoas que são normais e a classe outlier as pessoas que possuem algum tipo de arritmia cardíaca. Para esta base, 17 atributos foram removidos por não agregarem nenhuma informação (a saber, att20, att68, att70, att84, att132, att133, att140, att142, att144, att146, att152, att157, att158, att165, att205, att265 e att275), especificamente todos esses 17 atributos possuem o valor 0 para todas as instâncias. Outros 3 atributos foram removidos por possuírem mais que $10 \%$ de valores faltantes (a saber, att11, att12 e att14) e 2 instâncias foram removidas (id 5 e id 242) por possuírem um valor faltante. Após o pré-processamento, esta base possui 259 atributos numéricos e 450 instâncias, divididas em 206 outliers (45.78\%) e 244 inliers (54.22\%). A base Arrhythmia original (sem a remoção de atributos e instâncias) foi mantida na coleção benchmark final, a qual possui 279 atributos numéricos e 452 instâncias, divididas em 207 outliers (45.8\%) e 245 inliers $(54.2 \%)$.

- Page Blocks: Esta base é relativa à classificação de blocos do layout de páginas de documentos. Em análise de documentos, separar o texto de figuras ou gráficos é um passo essencial. Caso o conteúdo do bloco seja texto, ele foi aqui rotulado como inlier, caso contrário foi rotulado como outlier. Esta base possui 10 atributos numéricos e 5473 instâncias, divididas em 560 outliers (10.23\%) e 4913 inliers (89.77\%). Importante ressaltar que esta base possui 80 instâncias que são duplicatas (50 outliers e 30 inliers).

- Spam Base: Esta base refere-se à classificação de um email em spam ou não spam. Os emails que são spam foram considerados outliers, já os emails que não são spam foram considerados inliers. Esta base possui 57 atributos numéricos e 4601 instâncias, divididas em 1813 outliers (39.4\%) e 2788 inliers (60.6\%). Importante ressaltar que esta base possui 394 instâncias que são duplicatas (134 outliers e 260 inliers).

- Heart Disease: Base com registros de pacientes que possuem ou não problemas cardíacos. Pacientes com problemas foram considerados outliers e pacientes sem problemas 
foram considerados inliers. Esta base possui 13 atributos numéricos e 270 instâncias, divididas em 120 outliers (44.44\%) e 150 inliers (55.56\%).

- Cardiotocography: Referente à classificação de ataques cardíacos, esta base possui 3 classes: normal, suspeito ou patológico. Os pacientes normais foram rotulados como inliers e os demais foram rotulados como outliers. Esta base possui 21 atributos numéricos e 2126 instâncias, divididas em 471 outliers (22.15\%) e 1655 inliers (77.85\%). Os atributos utilizados foram os mesmos utilizados por Sundar et al. (2013). Importante ressaltar que esta base possui 12 instâncias que são duplicatas ( 5 outliers e 7 inliers).

- Internet Ads: Esta base representa um conjunto imagens na web. As instâncias que são propagandas foram consideradas outliers e as outras foram consideradas inliers. Para esta base, 3 atributos foram removidos por possuírem mais de $10 \%$ de valores faltantes (a saber, att1, att2 e att3). Com a remoção destes 3 atributos, todos os 1555 atributos restantes são binários, ou seja, seus valores são 0 ou 1 para todas as instâncias. Por isto, esta base não possui uma versão "normalizada", já que em sua versão original seus atributos possuem valores normalizados. Importante ressaltar que nesta base foram removidas 15 instâncias por possuírem valores faltantes para o atributo "att4" (a saber, id 133, id 230, id 231, id 232, id 233, id 643, id 1069, id 1084, id 1100, id 1317, id 1328, id 1354, id 1552, id 1882 e id 2033), sendo 5 delas outliers e 10 inliers. Após o pré-processamento, esta base possui 1555 atributos numéricos e 3264 instâncias, divididas em 454 outliers (13.91\%) e 2810 inliers (86.09\%). Esta base possui 1298 instâncias que são duplicatas (86 outliers e 1212 inliers). A base Internet Ads original (sem a remoção de atributos e instâncias) foi mantida na coleção benchmark final, a qual possui 1558 atributos numéricos e 3279 instâncias, divididas em 459 outliers (14\%) e 2820 inliers (86\%). Devido à altíssima dimensionalidade e à grande quantidade de duplicatas, particularmente para esta base apenas a versão sem duplicatas foi adicionada ao benchmark e, consequentemente, teve seus resultados analisados na próxima seção.

- Parkinson: Esta base refere-se à classificação de um paciente como saudável ou como portador da doença de Parkinson. Pessoas com a doença de Parkinson foram aqui rotuladas como outliers e pessoas saudáveis foram aqui rotuladas como inliers. Esta base possui 22 atributos numéricos e 195 instâncias, divididas em 147 outliers (75.39\%) e 48 inliers $(24.61 \%)$.

- Hepatitis: Esta base é relativa à predição de um paciente que possui hepatite entre morrer ou sobreviver. Pessoas que possuem hepatite e irão morrer foram aqui consideradas como outliers e pessoas que possuem hepatite e irão sobreviver foram aqui rotuladas como inliers. Essa rotulação foi atribuída após verificar que entre 480 e 520 milhões de pessoas possuem hepatite no mundo e 1 milhão de pessoas morrem por ano por causa desta doençẫ Esta base possui 19 atributos numéricos e 80 instâncias, divididas em 13 outliers

${ }^{2}$ Informação relacionada ao ano de 2010 e disponível no link: http://apps.who.int/gb/ebwha/ pdf_files/WHA63/A63_15-en.pdf 
$(16.25 \%)$ e 67 inliers $(83.75 \%)$.

- Wilt: Base com registros de árvores doentias e de outras coberturas terrestres. As árvores doentes foram aqui consideradas como outliers e as outras coberturas vegetais foram aqui rotuladas como inliers. Esta base possui 5 atributos numéricos e 4839 instâncias, divididas em 261 outliers (5.39\%) e 4578 inliers (94.61\%). Importante ressaltar que esta base possui 20 instâncias que são duplicatas (4 outliers e 16 inliers).

- Stamps3: Esta base contém registros de carimbos forjados (fotocopiada e/ou impresso) em meio a carimbos autênticos (de tinta), baseado nas propriedades e cores da impressora. Os carimbos forjados foram determinados aqui outliers e os carimbos verdadeiros inliers. Esta base possui 9 atributos numéricos e 340 instâncias, divididas em 31 outliers $(9.12 \%)$ e 309 inliers $(90.88 \%)$.

A Tabela 4.1 sumariza o que foi descrito acima em relação às bases coletadas até o momento que possuem uma semântica bem definida entre outliers e inliers. Dado que muitas bases apresentadas na Tabela 4.1 possuem um grande número de outliers, um procedimento realizado neste trabalho foi, além de manter versões de tais bases com a proporção original entre outliers e inliers, gerar versões modificadas aplicando uma subamostragem (conforme mencionado na Subseção 3.3.2.4). Tal subamostragem foi realizada reduzindo a quantidade de outliers para $20 \%, 10 \%, 5 \%$ e $2 \%$ da base. Entretanto, as bases Parkinson e Hepatitis não terão a versão com $2 \%$, pois a sua versão com $5 \%$ já conta com apenas 2 outliers. As avaliações nestas bases foram realizadas reportando média e desvio padrão de todas as 10 variantes subamostradas de cada base.

Tabela 4.1: Bases coletadas com semântica.

\begin{tabular}{|c|c|c|c|c|}
\hline Bases de Dados & Atributos & Outliers & Inliers & Instâncias \\
\hline Annthyroid & 21 & 534 & 6666 & 7200 \\
\hline Pima & 8 & 268 & 500 & 768 \\
\hline Arrhythmia & 259 & 206 & 244 & 450 \\
\hline Page Blocks & 10 & 560 & 4913 & 5473 \\
\hline Spam Base & 57 & 1813 & 2788 & 4601 \\
\hline Heart Disease & 13 & 120 & 150 & 270 \\
\hline Cardiotocography & 21 & 471 & 1655 & 2126 \\
\hline Internet Ads & 1555 & 454 & 2810 & 3264 \\
\hline Parkinson & 22 & 147 & 48 & 195 \\
\hline Stamps & 9 & 31 & 309 & 340 \\
\hline Hepatitis & 19 & 13 & 67 & 80 \\
\hline Wilt & 5 & 261 & 4578 & 4839 \\
\hline
\end{tabular}

${ }^{3}$ Esta base está disponível no link http:/ / cs.au.dk/ barbora/outDet . html e foi utilizada em Micenková et al. (2012) 


\subsubsection{Bases de Dados Utilizadas na Literatura Independente da Se- mântica}

A lista a seguir é uma compilação das bases de dados utilizadas na literatura independente da semântica e foram, em sua maioria, coletadas do repositório UCI (Frank and Asuncion, 2010). Caso não tenha sido mencionado pelos autores que utilizaram cada uma das bases, nas referências originais, qual subconjunto foi utilizado (treinamento ou teste), tais bases serão representadas através da unificação destes subconjuntos. Caso contrário, utiliza-se de acordo com a referência global.

- Pen-Based Recognition of Handwritten Digits: Esta base consiste na representação de dígitos escritos manualmente. As 10 classes presentes nesta base correspondem aos dígitos de 0 a 9 . A classe 4 foi definida como outlier e subamostrada para apenas 20 objetos por Schubert et al. (2012) e Kriegel et al. (2011). Conforme descrito anteriormente, a subamostragem é realizada 10 vezes e os resultados expressos em termos de média e desvio padrão. Após o pré-processamento, esta base possui 16 atributos numéricos e 9868 instâncias, divididas em 20 outliers $(0.2 \%)$ e 9848 inliers (99.8\%). Pelo fato desta base possuir todos os 16 atributos distribuídos no mesmo intervalo $[0,100]$, apenas a versão normalizada foi mantida (visto que a versão sem normalização e normalizada neste caso são semelhantes).

- Waveform: Esta base possui 3 classes de ondas. A classe 0 é definida como classe outlier e subamostrada para 100 objetos, assim como realizado por Zimek et al. (2013b). Os resultados para esta base são apresentados pela média e o desvio padrão de 10 execuções. Após o pré-processamento, esta base possui 21 atributos numéricos e 3443 instâncias, divididas em 100 outliers (2.9\%) e 3343 inliers (97.1\%).

- Shuttle: Esta base está relacionada ao tipo de dispositivo utilizado no controle de fluxo de fluídos em aeronaves. Zhang et al. (2009) utilizam as classes 1, 3, 4, 5, 6 e 7 como inliers e a classe 2 como outlier. Para executar seus experimentos, Zhang et al. (2009) fazem uma seleção de 1000 inliers para se juntar aos 13 outliers (Classe 2), formando uma base de dados com 1013 instâncias e duas classes (outlier e inlier). A seleção dos 1000 inliers realizada por Zhang et al. (2009) foi ralizada aqui 10 vezes, selecionando em cada execução diferentes inliers. Zhang et al. (2009) mencionam que utilizam apenas o conjunto de teste para esta base, portanto somente este conjunto foi mantido aqui. Os resultados para esta base são apresentados pela média e o desvio padrão de todas estas execuções. Por fim, esta base de dados possui 9 atributos e 1013 instâncias, divididas em 13 outliers $(1.28 \%)$ e 1000 inliers $(98.72 \%)$.

- Wisconsin Breast Cancer (WBC): Esta base de dados possui registros clínicos de câncer benigno ou maligno. Schubert et al. (2012) define os pacientes que possuem o câncer benigno como inliers e os que possuem o câncer maligno como outliers. Foi realizada uma subamostragem, removendo vários outliers, assim como realizado por Schubert et al. 
(2012), deixando apenas 10. Foram removidos 229 outliers para a base com duplicatas e 226 outliers para a base sem duplicatas. A subamostragem foi realizada aqui 10 vezes, selecionando em cada execução diferentes outliers. Os resultados para esta base são apresentados pela média e desvio padrão de todas as execuções. Para esta base foram removidas 16 instâncias que tinham um atributo com valor faltante (a saber, id 24, id 41, id 140, id 146, id 159, id 165, id 236, id 250, id 276, id 293, id 295, id 298, id 316, id 322, id 412 e id 618), sendo 2 instâncias outliers e 14 inliers. Após o pré-processamento, esta base possui 9 atributos numéricos e 454 instâncias, divididas em 10 outliers (2.2\%) e 444 inliers (97.8\%). Importante ressaltar que esta base possui 234 instâncias que são duplicatas (231 inliers e 3 outliers).

- Wisconsin Diagnostic Breast Cancer (WDBC): Base de dados médica, referente à extração de características nucleares para diagnóstico de câncer de mama. Os pacientes com câncer benigno foram considerados inliers e aqueles com câncer maligno foram considerados outliers segundo Zhang et al. (2009). Esta base foi pré-processada conforme realizado por Zhang et al.(2009), na qual os autores realizam uma subamostragem nos outliers, restando apenas 10. Tal subamostragem foi realizada aqui 10 vezes, selecionando em cada execução diferentes outliers. Os resultados para esta base são apresentados pela média e o desvio padrão de todas estas execuções. Após o pré-processamento, esta base possui 30 atributos numéricos e 367 instâncias, divididas em 10 outliers (2.72\%) e 357 inliers $(97.28 \%)$.

- KDDCup994: Esta base contém registros referentes a intrusões ou ataques simulados em rede de computadores. A versão utilizada neste trabalho segue o pré-processamento realizado em Nguyen and Gopalkrishnan (2010), na qual os autores utilizam a classe U2R como outlier e a classe normal como inlier. A classe U2R mencionada por Nguyen and Gopalkrishnan (2010) contém os seguintes ataques: buffer_overflow, ftp_write, imap, loadmodule, multihop, nmap, perl, phf, pod, rootkit e teardrop. Tais ataques possuem 246 instâncias da base de dados, definidos como outliers. Esta base possui 38 atributos numéricos, 3 atributos categóricos e 60839 instâncias, divididas em 60593 inliers e 246 outliers. Importante mencionar que esta base possui 12726 instâncias que são duplicatas (46 outliers e 12680 inliers). Foram utilizadas as três abordagens para trabalhar com os atributos categóricos desta base, assim como descritos na Seção 3.3.2.2.

- Lymphography: Base de pacientes de acordo com seus resultados de exames radiológicos com contraste do Sistema Linfático. Esta base consiste de quatro classes, sendo duas delas representadas por poucas instâncias (normal find e fibrosis). Tais classes foram agrupadas e consideradas aqui como outliers. Este procedimento foi realizado inspirado nos trabalhos realizados por Lazarevic and Kumar (2005), Nguyen et al. (2010), Zimek et al. (2013b) e He et al. (2005). Esta base possui 3 atributos numéricos, 16 atributos

\footnotetext{
${ }^{4}$ Esta base está disponível no repositório UCI através do arquivo corrected.gz
} 
categóricos e 148 instâncias, divididas em 6 outliers (4.05\%) e 142 inliers (95.95\%). Foram utilizadas as três abordagens para trabalhar com os atributos categóricos desta base, assim como descritos na Seção 3.3.2.2.

- Amsterdam Library of Object Images (ALOI): Esta base é uma coleção de imagens disponíveis para pesquisas científicas. O procedimento realizado nesta base foi inspirado nos trabalhos realizados por Schubert et al. (2012) ${ }^{5}$ e Kriegel et al. (2011). Esta base possui 27 atributos numéricos (descritos no espaço Hue-Saturation-Brightness (HSB)) e 50000 instâncias, divididas em 1508 outliers (3.04\%) e 48492 inliers (96.98\%). Importante ressaltar que esta base possui 466 instâncias que são duplicatas e todas pertencem aos inliers.

- Wisconsin Prognostic Breast Cancer (WPBC): A versão utilizada neste trabalho foi a disponibilizada por Keller et al. (2012) em que se utiliza a classe 'R' (minoritária) como outlier e a classe ' $N$ ' como inlier. Ao disponibilizar esta base de dados, Keller et al. (2012) aplicou uma normalização nos dados como pré-processamento e que, portanto, também foi aplicada neste trabalho. Após o pré-processamento, esta base possui 33 atributos numéricos e 198 instâncias, divididas em 47 outliers (23.74\%) e 151 inliers (76.26\%).

- Glass: Esta base refere-se à classificação de tipos de vidro, motivada por investigações criminológicas. A versão utilizada neste trabalho foi a disponibilizada por Keller et al. $(2012)^{5}$, em que se utiliza a classe 6 (minoritária) como outlier e todas as outras classes como inliers. Ao disponibilizar esta base de dados, Keller et al. (2012) aplicou uma normalização nos dados como pré-processamento e que, portanto, também foi aplicada neste trabalho. Após o pré-processamento, esta base possui 7 atributos numéricos e 214 instâncias, divididas em 9 outliers (4.21\%) e 205 inliers (95.79\%). Esta base possui apenas 1 duplicata, portanto não foi criada uma nova versão sem duplicatas.

- Ionosphere: Esta base contém registros de radares bons, os quais mostram evidências de algum tipo de estrutura na ionosfera, e radares ruins, os quais não captam os sinais de interesse. A versão utilizada neste trabalho foi a disponibilizada por Keller et al. $(2012)^{5}$, em que se utiliza a classe 'b' (minoritária) como outlier e a classe 'g' como inlier. Keller et al. (2012) removeu os atributos 1 e 2 de sua base de dados. Ao disponibilizar esta base de dados, Keller et al. (2012) aplicou uma normalização nos dados como pré-processamento e que, portanto, também foi aplicada neste trabalho. Após o pré-processamento, esta base possui 32 atributos numéricos e 351 instâncias, divididas em 126 outliers (35.9\%) e 225 inliers $(64.1 \%)$. Esta base possui apenas 1 duplicata, portanto não foi criada uma nova versão sem duplicatas.

${ }^{5}$ Esta base está disponível através do link: http://elki.dbs.ifi.lmu.de/wiki/DataSets/ MultiView

${ }^{\circ}$ Base disponível através do link: http://www.ipd.kit.edu/ muellere/HiCS/ 
A Tabela 4.2 sumariza o que foi descrito acima em relação às bases coletadas até o momento dentre aquelas utilizadas na literatura independentemente da semântica entre outliers e inliers.

Tabela 4.2: Bases coletadas da literatura.

\begin{tabular}{|c|c|c|c|c|}
\hline Bases de Dados & Atributos & Outliers & Inliers & Instâncias \\
\hline WBC & 9 & 10 & 444 & 454 \\
\hline WDBC & 30 & 10 & 357 & 367 \\
\hline KDDCup99 & 41 & 246 & 60593 & 60839 \\
\hline Pen Digits & 16 & 20 & 9848 & 9868 \\
\hline Waveform & 21 & 100 & 3343 & 3443 \\
\hline Lymphography & 19 & 6 & 142 & 148 \\
\hline ALOI & 27 & 1508 & 48492 & 50000 \\
\hline Shuttle & 9 & 13 & 1000 & 1013 \\
\hline WPBC & 33 & 47 & 151 & 198 \\
\hline Glass & 7 & 9 & 205 & 214 \\
\hline Ionosphere & 32 & 126 & 225 & 351 \\
\hline
\end{tabular}

Diversas bases utilizadas neste projeto foram também exploradas neste contexto de detecção de outliers por outros autores além daqueles explicitamente citados acima. A Tabela 4.3 relaciona as bases geralmente utilizadas nesta área com artigos relevantes e os respectivos préprocessamentos realizados em cada uma delas. Considerando o conceito subjetivo de outliers, uma base pode ser pré-processada de várias maneiras diferentes, o que também é discutido a seguir.

A base WBC foi pré-processada de 4 maneiras distintas por diferentes autores. Schubert et al. (2012) e Kriegel et al. (2011) realizam uma subamostragem da classe "malignant" (outlier), deixando apenas 10 outliers nos dados. Já em Zimek et al. (2013b), é mencionado a realização de uma subamostragem na classe "malignant", porém sem mencionar quantos outliers foram mantidos. No trabalho de Gao and Tan (2006), a classe "malignant" foi definida como outlier e a classe "benign" como inlier, sem realizar qualquer tipo de subamostragem. Por fim, He et al. (2005) realizam uma subamostragem na classe "malignant", mantendo 39 outliers ao invés de 10.

A base KDDCup99 é bastante conhecida e utilizada na área de detecção de anomalias. Schubert et al. (2012) mencionam a utilização desta base com 60839 objetos e 38 dimensões numéricas em seus estudos, porém não informam qual o respectivo pré-processamento utilizado. Nenhuma informação sobre o pré-processamento utilizado nesta base foi disponibilizado no trabalho de Kriegel et al. (2011). Já os trabalhos de Nguyen et al. (2010), Lazarevic and Ku$\operatorname{mar}$ (2005) e Nguyen and Gopalkrishnan (2010) utilizam a classe U2R como outlier e a classe Normal como inlier. A classe U2R (a saber, buffer_overflow, loadmodule, perl e rootkit) possui 39 instâncias representativas na base de dados disponível. Entretanto, Lazarevic and Kumar (2005) mencionam que existem 246 outliers presentes na base. 246 é exatamente o número de instâncias que contêm os seguintes ataques: buffer_overflow, ftp_write, imap, loadmodule, 
multihop, nmap, perl, phf, pod, rootkit e teardrop (Nguyen and Gopalkrishnan, 2010), o que sugere uma inconsistência no texto de Lazarevic and Kumar (2005). Importante mencionar que Nguyen and Gopalkrishnan (2010) removem os atributos categóricos presentes na base e Lazarevic and Kumar (2005) transformam-os em numéricos utilizando a medida IDF,

A base Pen Digits foi utilizada nos trabalhos de Schubert et al.(2012) e Kriegel et al.(2011), os quais subamostram a classe 4 (definida por eles como outliers) para apenas 20 instâncias. Já Keller et al. (2012) subamostram a classe 0 para $10 \%$ de sua quantidade original, tornando tal classe outlier.

O pré-processamento realizado por Zimek et al. (2013b) na base Waveform foi subamostrar a classe 0 (definida por eles como outlier), porém sem mencionar quantos outliers permaneceram na base final.

Segundo a descrição da base Lymphography em Nguyen et al. (2010) e Lazarevic and Kumar (2005), os autores unem as classes 2 e 4 (que segundo esses autores seriam as minoritárias), e definem tal união como outliers. Entretanto, as classes 1 e 4 é que na verdade são minoritárias nesta base, conforme mencionado por Zimek et al. (2013b) e He et al. (2005). Nguyen et al. (2010) e Lazarevic and Kumar (2005) unem as classes 2 e 4 (outliers) e mencionam que tal procedimento deixa a base Lymphography com 6 outliers, mas esta quantidade de outliers é na verdade alcançada quando se une as classes 1 e 4, como realizado por Zimek et al. (2013b) e He et al. (2005).

A base ALOI é utilizada nos trabalhos de Schubert et al. (2012) e Kriegel et al. (2011), e ambos utilizam a versão com 50000 objetos (1508 outliers) e 27 dimensões, indicadas pelo espaço HSB. de Vries et al. (2012) também utilizam a base ALOI porém com outro préprocessamento, utilizando um espaço de atributos diferente. Tais autores representam 1000 objetos distintos com 24 imagens cada, tiradas com 8 diferentes orientações sobre 3 diferentes direções de iluminação. Todos estes objetos estão representados em escala de cinzas com resoluções 192x144, representados por 27648 dimensões (valores de píxeis).

Shuttle é outra base bastante utilizada no contexto de detecção de anomalias. Lazarevic and Kumar (2005), Nguyen et al. (2010) e Abe et al. (2006) transformam esta base em 5 versões diferentes. Cada versão possui uma das classes 2, 3, 5, 6 ou 7 como outlier e a classe 1 como inlier. Já Gao and Tan (2006) consideram as classes 1 e 4 como inliers e adicionam uma subamostra das classes 2, 3, 5, 6 e 7 como outliers, porém sem mencionar a quantidade de outliers adicionados. Outro trabalho importante na área que utiliza esta base foi realizado por Zhang et al. (2009). Neste trabalho, os autores definem a classe 2 como outlier e realizam uma subamostragem de 1000 objetos das classes remanescentes para compor os inliers.

O pré-processamento realizado por Zhang et al. (2009) na base WDBC foi apenas definir a classe ' $M$ ' (malignant) como outlier e subamostrá-la para apenas 10 instâncias. Keller et al. (2012) e Nguyen et al. (2010) utilizam o mesmo pré-processamento porém sem subamostrar a classe malignant.

Para as bases WPBC, Glass e Ionosphere, Keller et al. (2012) utilizam a classe minoritária como outlier e as classes remanescentes como inliers. 
Tabela 4.3: Relação entre bases utilizadas na literatura, artigos importantes na área e o préprocessamento realizado.

\begin{tabular}{|c|c|c|}
\hline Bases de Dados & Artigos & Pré-processamento \\
\hline \multirow{5}{*}{ WBC } & Schubert et al. (2012) & Subamostragem 'malignant' / 10 (out.) \\
\hline & Kriegel et al. (2011) & Subamostragem 'malignant' / 10 (out.) \\
\hline & Zimek et al. (2013b) & Subamostragem 'malignant' \\
\hline & Gao and Tan (2006) & 'malignant'(out.) vs ‘benign'(in.) \\
\hline & He et al. $(2005)$ & Subamostragem 'malignant' / 39 (out.) \\
\hline \multirow{5}{*}{ KDDCup'99 } & Schubert et al. (2012) & 60839 obj. e 38 dimensões numéricas \\
\hline & Kriegel et al. (2011) & - \\
\hline & Nguyen et al. (2010) & U2R(out.) vs normal(in.) \\
\hline & Lazarevic and Kumar (2005) & $\mathrm{U} 2 \mathrm{R}($ out.) vs normal(in.) \\
\hline & Nguyen and Gopalkrishnan (2010) & U2R(out.) vs normal(in.) \\
\hline \multirow{3}{*}{ Pen Digits } & Keller et al. $(2012)$ & Subamostragem '0’ / 10\% (out.) \\
\hline & Schubert et al. (2012) & Subamostragem ‘4' / 20 out. \\
\hline & Kriegel et al. (2011) & Subamostragem '4' / 20 out. \\
\hline Waveform & Zimek et al. (2013b) & Subamostragem '0' \\
\hline \multirow{4}{*}{ Lymphography } & Nguyen et al. (2010) & Classes 2 e 4 (out.) vs outras (in.) \\
\hline & Lazarevic and Kumar (2005) & Classes 2 e 4 (out.) vs outras (in.) \\
\hline & Zimek et al. (2013b) & Classes 1 e 4 (out.) vs outras (in.) \\
\hline & He et al. (2005) & Classes 1 e 4 (out.) vs outras (in.) \\
\hline \multirow{3}{*}{ ALOI } & Schubert et al. (2012) & 50000 obj., 27 dimensões e 1508 out. \\
\hline & Kriegel et al. (2011) & 50000 obj., 27 dimensões e 1508 out. \\
\hline & de Vries et al. (2012) & 24000 imagens, 27648 dimensões \\
\hline \multirow{5}{*}{ Shuttle } & Lazarevic and Kumar (2005) & Classes 2, 3, 5, 6, 7 (out.) vs classe 1 (in.) \\
\hline & Nguyen et al. (2010) & Classes 2, 3, 5, 6, 7 (out.) vs classe 1 (in.) \\
\hline & Abe et al. (2006) & Classes 2, 3, 5, 6, 7 (out.) vs classe 1 (in.) \\
\hline & Zhang et al. (2009) & Classe 2 (out.) vs Sub. outras / 1000 (in.) \\
\hline & Gao and Tan (2006) & Sub. classes $2,3,5,6,7$ (out.) vs outras (in.) \\
\hline \multirow{3}{*}{ WDBC } & Nguyen et al. (2010) & 'malignant' (out.) vs 'benign' (in.) \\
\hline & Zhang et al. (2009) & Subamostragem 'malignant' / 10 (out.) \\
\hline & Keller et al. (2012) & 'malignant' (out.) vs ‘benign' (in.) \\
\hline WPBC & Keller et al. (2012) & Classe 'R' (out.) vs classe 'N' (in.) \\
\hline Glass & Keller et al. (2012) & Classe 6 (out.) vs outras (in.) \\
\hline Ionosphere & Keller et al. (2012) & Classe 'b' (out.) vs classe 'g' (in.) \\
\hline
\end{tabular}

\subsection{Execução de Algoritmos de Detecção de Outliers}

Após selecionar e pré-processar as bases descritas na Seção 4.1, os algoritmos de detecção de outliers mencionados no Capítulo 2 foram aplicados com o objetivo de comparar os algo- 
ritmos aplicados e avaliar as bases de dados como candidatas a fazerem parte de uma coleção benchmark.

Os algoritmos utilizados possuem apenas um parâmetro $(k)$, o qual está relacionado com a vizinhança que será considerada para obter o score de outlier para cada instância. O parâmetro $k$ foi variado no intervalo [1,100]. Com isso, 100 valores de $k$ foram utilizados para cada algoritmo. Todos os algoritmos foram utilizados conforme implementados na plataforma ELKI (Achtert et al. 2013), utilizando a distância Euclidiana. Os valores de $k$ mencionados se referem ao respectivo parâmetro para cada método, de acordo com a versão 0.6.5 do ELKI.

Já que possuímos um total de 12 algoritmos, cada qual sendo executado para 100 valores do parâmetro de vizinhança (a menos que alguma base de dados possua menos de 100 instâncias) em 23 bases de dados (para as quais podem haver diversas variações devido à normalização, presença de duplicatas, tratamento de atributos categóricos e subamostragem realizadas 10 vezes em variadas taxas), obtemos um total de 1.268 .576 experimentos. Os resultados completos, incluindo todas as figuras para todas as bases de dados e algoritmos estão disponíveis em nosso repositório on-line mencionado no início deste capítulo.

\subsubsection{Caracterização dos Métodos de Acordo com os Resultados}

Nesta seção vamos discutir os resultados coletados relacionados com a qualidade dos métodos de detecção de anomalias.

A Figura 4.1 ilustra, para algumas bases de dados, o comportamento dos resultados obtidos para cada algoritmo, considerando os métodos de avaliação $\mathrm{AUC}, P @ \mathrm{e}$ e $\mathrm{AP}$. Importante mencionar que nesta Figura 4.1 estão os resultados no decorrer de todos os 100 valores de $k$. Através dos resultados é possível perceber o porquê da medida AUC ser mais popular: ela tende a atingir valores melhores em comparação com $P @ n$ ou $\mathrm{AP}$. As razões para esta diferença situam-se no fato de que a medida AUC trata o desbalanço de classes naturalmente e é uma medida da qualidade do ranking geral. $P @ n$ leva em consideração quantos verdadeiros outliers estão no topo (dependendo da escolha de $n$ ). Portanto, esta medida pode ser útil para discernir a qualidade de vários resultados que possuem desempenhos próximos em termos de AUC, o que foi observado anteriormente por Davis and Goadrich (2006). Por outro lado, a medida AP segue mais o comportamento da AUC, isso porque ele acessa o ranking de todos os outliers, mas, similar à $P @ n, \mathrm{AP}$ também tende a gerar valores menores para problemas desbalanceados.

Os resultados das métricas AdjustedP@n Adjusted $P$ AP seriam as mesmas curvas presentes na Figura 4.1, apenas deslocadas no eixo y. O ajuste será interessante apenas se compararmos o comportamento entre diferentes bases de dados, pois, nesse caso, os ajustes consideram diferentes quantidades de outliers.

A primeira questão que buscamos quando se trata de uma pesquisa de comparação entre diferentes métodos de detecção de anomalias é perguntar se alguns métodos superam de forma consistente outros métodos. Para realizar tal comparação, foi realizada aqui uma contagem simples dos melhores métodos (maior AUC), para cada valor de $k$ e cada base de dados. As figuras na coluna da esquerda da Figura 4.2 estão relacionadas com a quantidade de vezes 

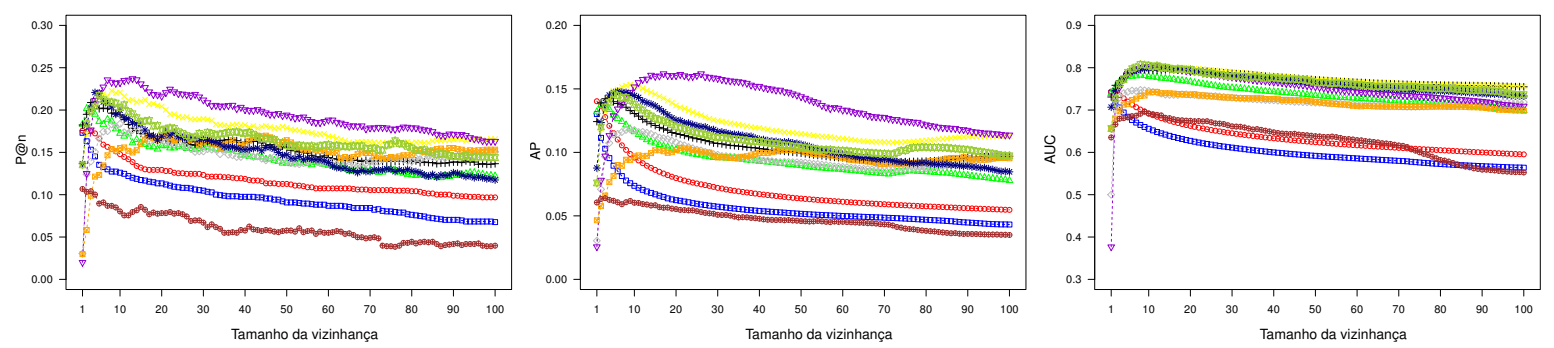

(a) ALOI (sem duplicatas, normalizada, sem subamostragem).
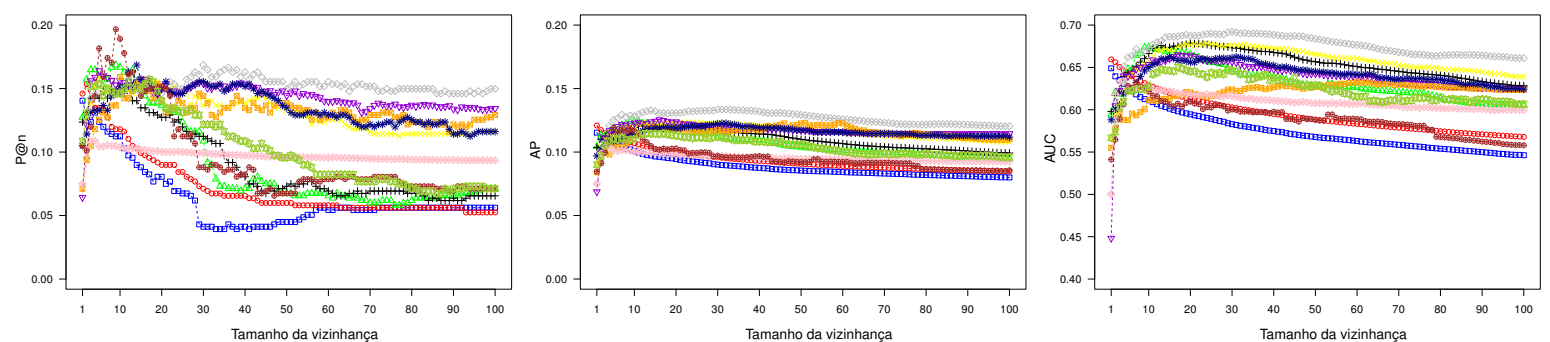

(b) Annthyroid (sem duplicatas, normalizada, sem subamostragem).
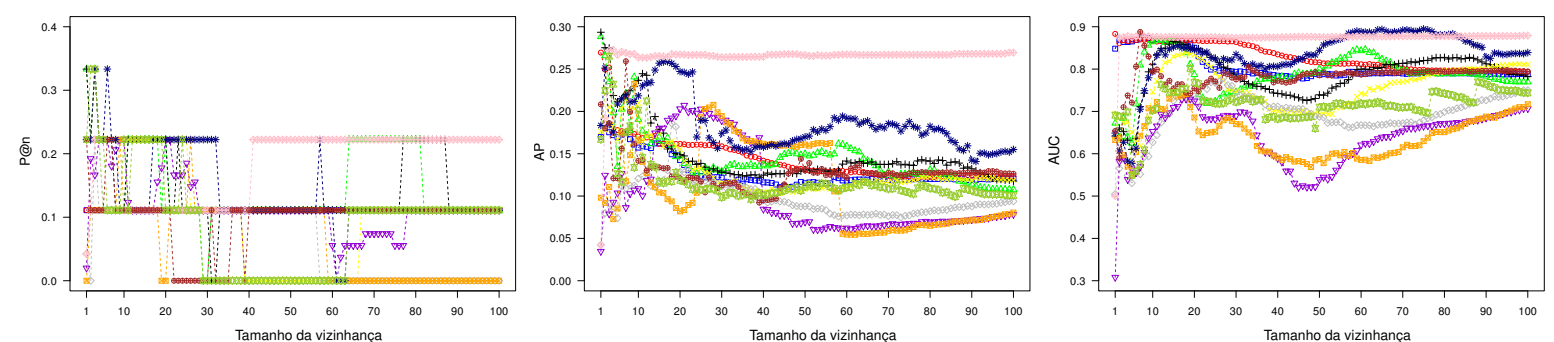

(c) Glass (sem duplicatas, normalizada, sem subamostragem).
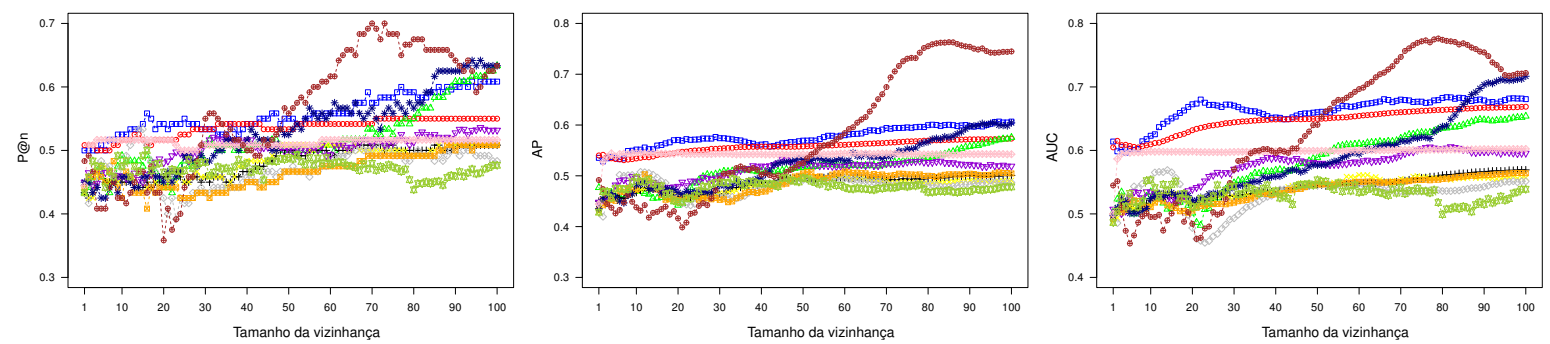

(d) HeartDisease (sem duplicatas, normalizada, sem subamostragem).
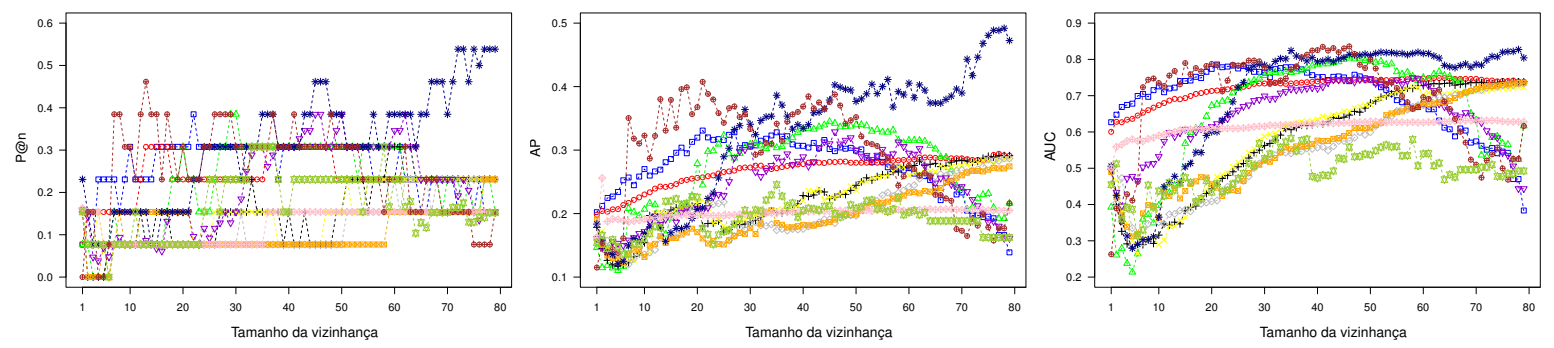

(e) Hepatitis (sem duplicatas, normalizado, sem subamostragem).

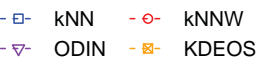
- LOF - + SimplifiedLOF
$\begin{array}{ll}x- & \text { LOOP } \\ -\rightarrow- & \text { LDF }\end{array}$
\&DOF
- INFLO

Figura 4.1: Resultados em algumas bases de dados comparando $P @$, $\mathrm{AP} \mathrm{e} \mathrm{AUC}$ 
que um determinado método foi classificado em primeiro, segundo ou terceiro considerando os valores de AUC Já as figuras na coluna da direita da Figura 4.2 representam a quantidade de vezes que um determinado método foi classificado entre os 4 melhores, 4 intermediários e 4 piores. A divisão entre as figuras (a), (b), (c) e (d) foi feita separando as bases de dados $\mathrm{com} / \mathrm{sem}$ duplicatas e com/sem normalização.

Considerando os 3 primeiros rankings (figuras à esquerda da Figura 4.2), os métodos globais $\mathrm{KNN}$ KNNW vencem frequentemente, seguidos do LOFe suas variantes mais próximas LDOF e LDF. Na a agregação dos rankings 1-4, 5-8 e 9-12 (figuras à direita da Figura 4.2), KNN $\mathrm{KNNW}$ estão entre os 4 primeiros com mais frequência do que os outros métodos. [LOF teve uma performance melhor do que suas variantes mais próximas neste tipo de agregação dos métodos. Ao avaliar em detalhes experimentos individuais, podemos perceber que é sempre possível encontrar para cada método uma configuração (parâmetro e base de dados) que supere os demais métodos, embora no geral alguns superem em centenas de experimentos enquanto outros em dezenas de milhares de experimentos.

Olhar o ranking relativo dos métodos para um valor fixo do parâmetro $k$ apenas explica parte do cenário sobre performance relativa dos métodos. Foi aplicado aqui neste trabalho também uma comparação entre os algoritmos de detecção de outliers analisando a qualidade alcançada comparando valores de $k$ possivelmente diferentes, como por exemplo a melhor performance de cada método (e.g., a performance adquirida quando o usuário pergunta para um oráculo o valor ótimo do parâmetro $k$ para cada método). Na Figura 4.3 estão representados para cada método a média dos valores de AUC (e desvio padrão) por todas as bases de dados (divididas em sem/com duplicatas e sem/com normalização) para o melhor valor de $k$ dentro do intervalo considerado. Ou seja, verificamos o desempenho máximo que cada método consegue alcançar em cada base de dados e realizamos a média para todas as bases de dados de uma certa categoria, conforme as subfiguras apresentadas.

Em relação às duplicatas e à normalização, os resultados dos melhores valores de AUC em média para todas as bases de dados foram adquiridas através da remoção das duplicatas e aplicação da normalização nos dados (Figura 4.3(c)).

Através dos resultados apresentados pela Figura 4.3, podemos verificar que dois métodos, Fas $\mathrm{ABOD}$ KDEOS, foram consideravelmente piores do que os outros métodos considerando os melhores desempenhos. O método KDEOS já possuía uma tendência a ser mais classificado como um dos últimos métodos (Figura 4.2) independente da remoção ou não das duplicatas e da normalização ou não dos dados. Já o método Fas ABOD também possuía uma tendência a estar entre os 4 últimos em bases de dados com duplicatas. É visível o quão este método não consegue trabalhar bem com duplicatas na base de dados. Entretanto, esses métodos possuem um parâmetro adicional além do $k$ que foi fixado em valor padrão sugerido pelos autores. Nas bases sem normalização, todos os outros resultados são similares, com LOF ligeiramente melhor do que os outros métodos. Já nas bases com normalização os métodos clássicos $\mathrm{KNN}$, KNNW LOF estão no topo.

Com os resultados apresentados até aqui, podemos concluir que nenhum dos métodos desen- 

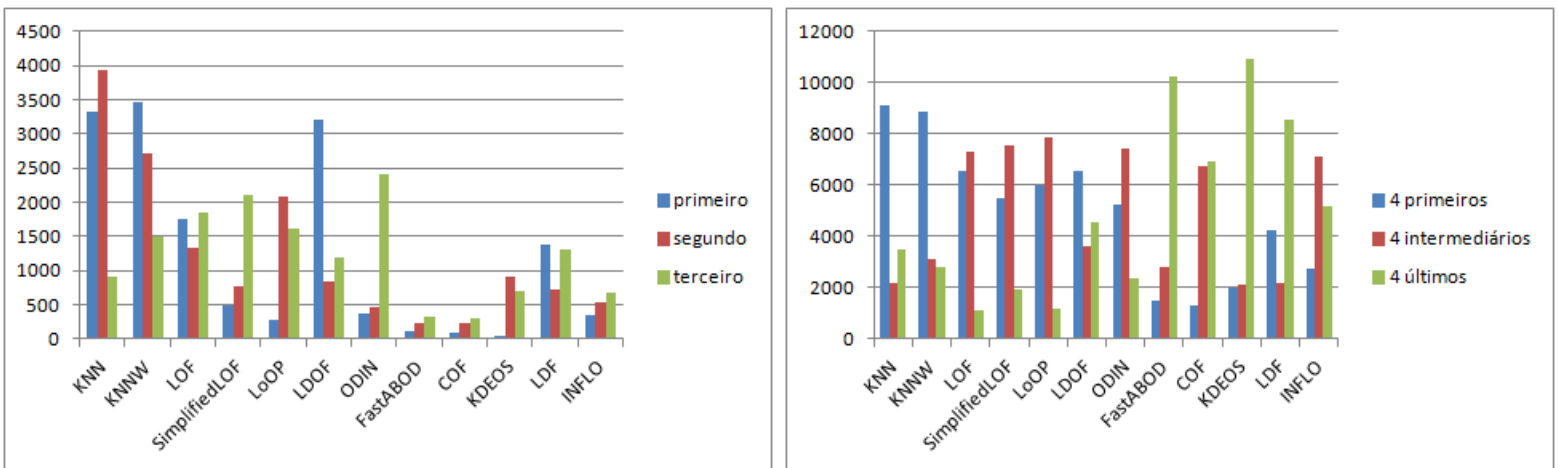

(a) Bases de dados com duplicatas e com normalização.
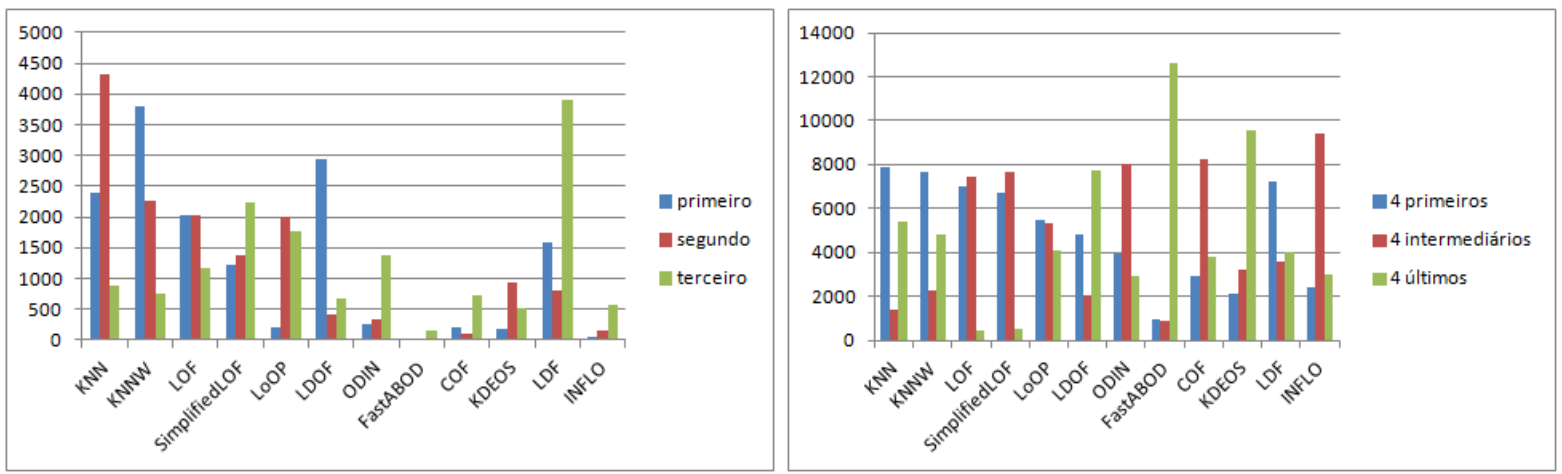

(b) Bases de dados com duplicatas e sem normalização.
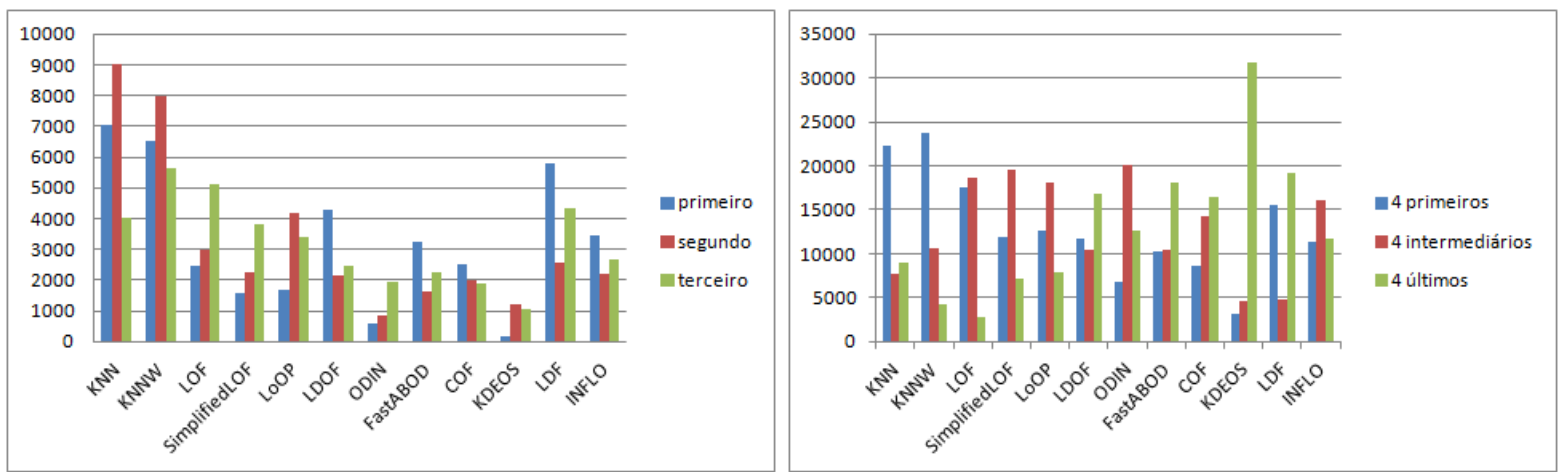

(c) Bases de dados sem duplicatas e com normalização.
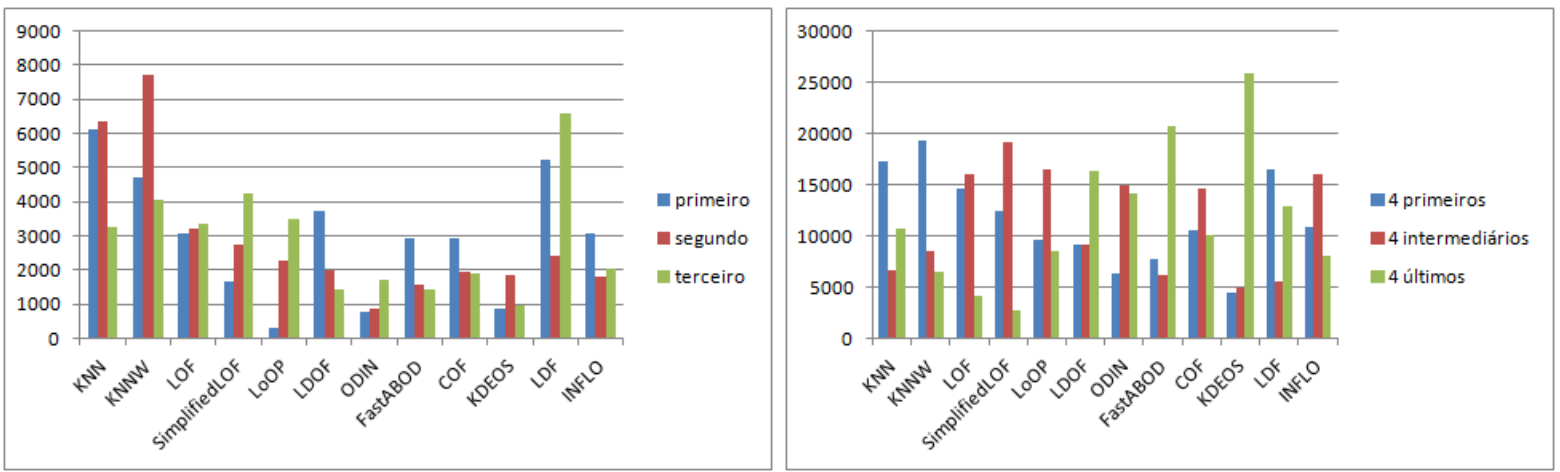

(d) Bases de dados sem duplicatas e sem normalização.

Figura 4.2: Ranking dos métodos pela performance considerando todos os valores de $k$ e todas as bases de dados. 


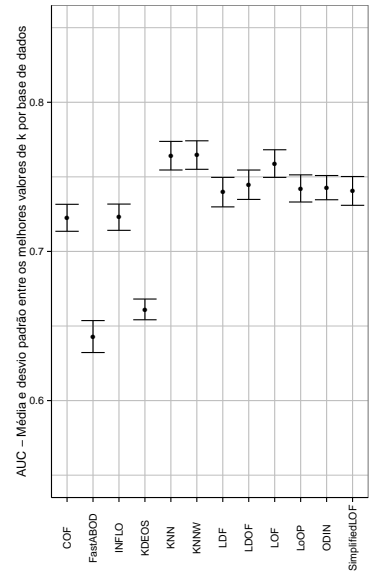

(a) Média e desvio padrão dos maiores valores de AUC por base de dados com duplicatas e com normalização.

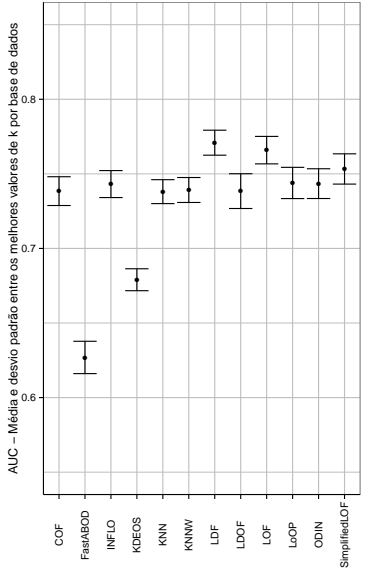

(b) Média e desvio padrão dos maiores valores de AUC por base de dados - AU com duplicatas e sem normalização.

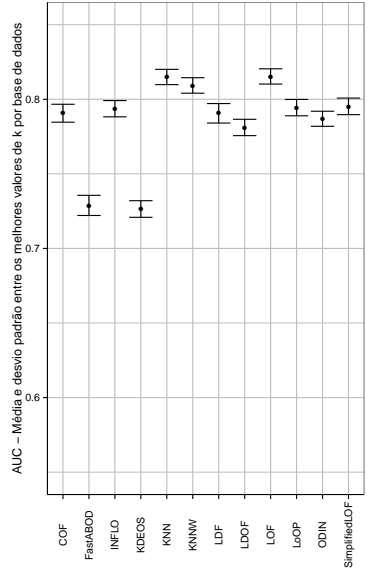

(c) Média e desvio padrão dos maiores valores de AUC por base de dados sem duplicatas e com normalização.

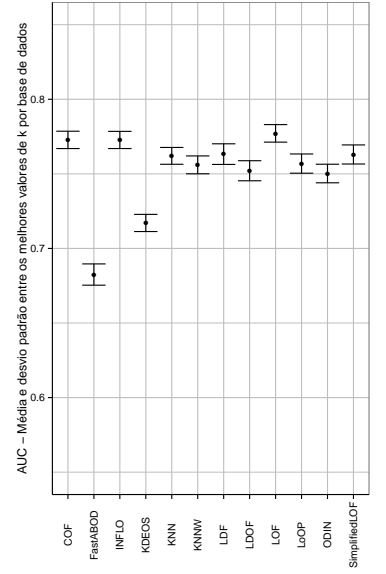

(d) Média e desvio padrão dos maiores valores de AUC por base de dados sem duplicatas e sem normalização.

Figura 4.3: Comparação dos melhores valores de AUC obtidos por método, considerando todas as bases de dados.

volvidos recentemente consegue sistematicamente ou significativamente superar o desempenho dos métodos clássicos (KNN, KNNW] e LOF), embora isso possa ocorrer pontualmente em bases de dados específicas. Estes resultados também trazem uma luz sobre como a avaliação de novos métodos devem ser realizadas. Sempre é possível encontrar casos (bases de dados, configurações específicas de parâmetros) nos quais um método em específico é melhor do que outros (como vimos na Figura 4.2). O comportamento de métodos que dependem de parâmetros deve, portanto, ser estudado com diferentes ajustes dos parâmetros, como demonstramos aqui, e pode-se esperar que os resultados para diferentes valores dos parâmetros podem ser bastante diferentes uns dos outros. Isto pode parecer senso comum, mas existem muitos trabalhos em que os autores apenas selecionam um valor para o parâmetro e comparam métodos baseados em tais escolhas arbitrárias (apenas para apontar alguns exemplos recentes, Keller et al. (2012), Liu et al. (2012), Müller et al. (2012), Müller et al. (2011), Ting et al. (2013)). Além disso, o comportamento dos métodos deve ser avaliado em uma coleção ampla e diversa de bases de dados, ao invés de um conjunto pequeno e arbitrariamente selecionado.

\subsubsection{Caracterização das Bases de Dados de Acordo com os Resulta- dos}

Nesta subseção vamos analisar as propriedades das bases de dados coletadas e discutir a viabilidade de uma futura coleção de bases de dados para detecção de outliers.

Primeiramente, para obter uma melhor compreensão da dificuldade relativa de diferentes bases de dados, vamos analisar o valor de desempenho agregado para cada versão de cada base de dados (agregado relativo a todos os métodos). Para esta finalidade, selecionamos para cada 

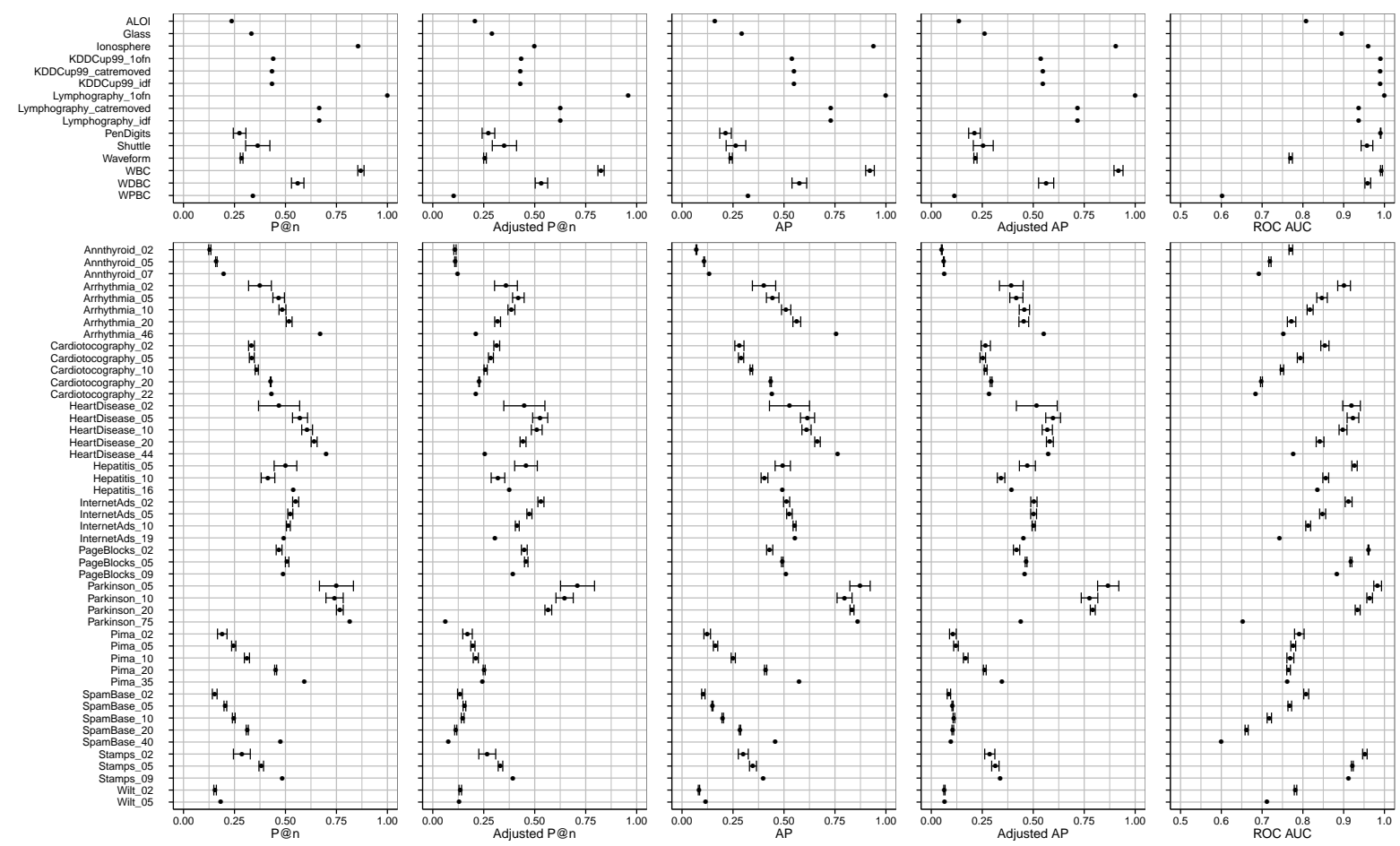

Figura 4.4: Comparação entre os melhores resultados alcançados por base de dados, considerando a versão normalizada e sem duplicatas.

método o melhor desempenho obtido em cada base de dados (i.e., aquele referente à escolha ótima do parâmetro $k$ ) e realizamos uma média entre todos os métodos. Nas bases de dados que possuem diferentes versões devido à subamostragem, os resultados foram apresentados reportando média e desvio padrão. A Figura 4.4 mostra em cada coluna os resultados de uma medida de desempenho diferente, separadamente para as bases de dados da Tabela 4.1 e da Tabela 4.2. Consideramos melhor analisar aqui os resultados das bases sem duplicatas e com normalização, pois estas representam os procedimentos mais utilizados na literatura para detecção de outliers. Entretanto, todos os outros resultados encontram-se no repositório on-line mencionado no início deste capítulo.

A primeira coluna da Figura 4.4 apresenta a precisão em $n(P @ n)$. Podemos perceber claramente que os métodos, em média, podem alcançar resultados bem melhores em algumas bases de dados do que outras. Por exemplo, nas bases Parkinson, os métodos alcançaram valores particularmente altos de $P @ n$, e as bases Annthyroid estão entre as bases de dados com menores valores desta medida. Em geral, parece haver uma certa tendência que os valores de $P @ n$ aumentem conforme aumenta o número de outliers na base de dados, especialmente quando se consideram as diferentes versões de uma mesma base com diferentes taxas de subamostragem. Assim como discutido anteriormente, um ranking aleatório possui um valor de $P @ n$ esperado de $|O| / N$, sem importar com o valor escolhido para $n$. Com isso, a medida em que aumenta a quantidade de outliers, o valor esperado de $\overline{P @ n}$ mesmo para um ranking aleatório aumenta. Por esta razão, introduzimos a medida ajustada da precisão em $n$ (Adjusted $P @ n$ ).

A segunda coluna da Figura 4.4 apresenta os valores da precisão em $n$ ajustada (Adjusted $(\overline{P @ n}$ ). A tendência agora quando aumenta a quantidade de outliers nas bases de 
dados é claramente atenuada ou até mesmo invertida em alguns casos, o que pode ser resultado da classe subamostrada (outliers) se parecer cada vez mais com um cluster do que um conjunto de outliers quando a subamostragem é baixa. Assim como $P @ n$, Adjusted $P @ n$ considera apenas as primeiras $n$ posições do ranking, o que pode conduzir a baixos valores (por exemplo Annthyroid, Pima e SpamBase) e pode não ser capaz de distinguir alguns rankings que são diferentes em termos de qualidade.

A terceira coluna da Figura 4.4 apresenta a precisão média ( $(\mathrm{AP})$. Esta medida tenta superar ao menos em parte o problema apresentado pela $P @ n$ de não considerar todo o ranking. Entretanto, a mesma sofre do mesmo problema apresentado pela $P @ n$ em respeito ao aumento no número de outliers nas bases de dados.

A quarta coluna da Figura 4.4 apresenta a precisão média ajustada (AdjustedAP). Novamente os valores ajustados são mais estáveis a medida em que a quantidade de outliers aumenta.

A quinta coluna da Figura 4.4 apresenta a área abaixo da curva ROC (AUC). Esta é a medida de qualidade mais utilizada para algoritmos de detecção de anomalias. A curva ROC considera todo o ranking ao gerar seus valores e os valores da AUC mostram o efeito negativo, em média, da performance dos métodos quando a porcentagem de outliers aumenta para uma base de dados. Este efeito também é claro para bases de dados nas quais a Adjusted $P @ n$ não conseguiu determinar a diferença, pois considerava apenas as $n$ primeiras posições, levando a valores pequenos da medida (e.g., SpamBase). Contudo, também é claro que valores altos de AUC em uma base de dados demonstram apenas que no ranking total, os outliers tendem a estar posicionados acima dos inliers (maior probabilidade), mas isto não significa que os outliers estão exatamente no topo do ranking. Para algumas bases de dados este pode ser o caso, mas para outras pode haver uma grande quantidade de inliers entre os $n$ primeiros no ranking, o que limita a utilidade dos resultados neste caso. Portanto, podemos concluir que as medidas AUC e Adjusted $P @ n$ se complementam, mostrando diferentes aspectos de uma solução, ambos relevantes em prática.

A Figura 4.5 apresenta rankings dos outliers obtidos pelos métodos em bases de dados com $3 \%$ a 5\% de outliers, que são aquelas mais compatíveis com a intuição que outliers devem ser observações mais raras. Para cada gráfico presente na Figura 4.5, o eixo y representa os verdadeiros outliers e o eixo $x$ os rankings dos melhores resultados para cada método. Os rankings foram agrupados e coloridos da seguinte forma: um outlier detectado entre os $n$ primeiros recebe o rank de 1 (azul escuro), um outlier não detectado entre os $n$ primeiros, mas detectado entre os $2 n$ primeiros recebe o rank de 2 (azul mais claro), ..., e um outlier detectado apenas entre os $10 n$ primeiros ou além, recebe o rank de 10 (vermelho escuro). O valor de $n$ para esse ranking é igual ao número de verdadeiros outliers em cada base de dados $(n=|O|)$. Portanto, em uma dada coluna, os outliers que possuírem a cor azul escura contribuíram para a medida $P @ n$. Os outliers foram ordenados ao longo do eixo $y$ de acordo com o rank médio dentre todos os métodos.

Visto que utilizamos, para cada método, o melhor resultado alcançado, esses gráficos nos informam menos sobre a performance geral de um método em uma base de dados, mas mais 

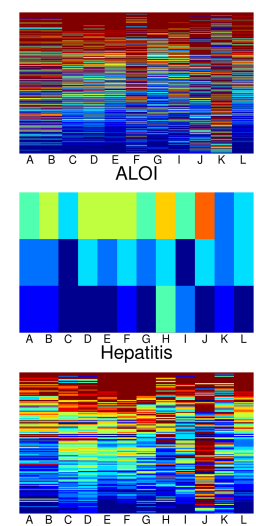

SpamBase
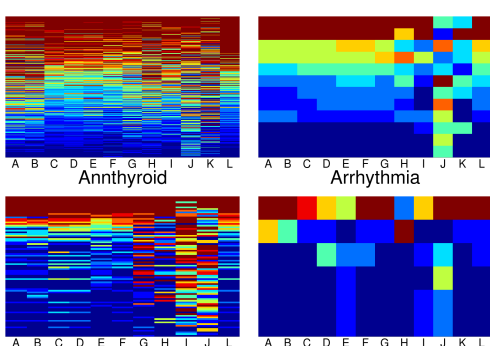

InternetÂds

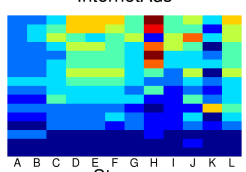

Stamps

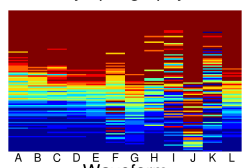

Waveform

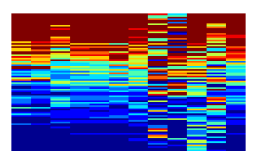

Cardiotocography

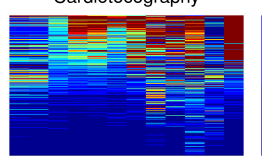

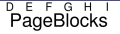

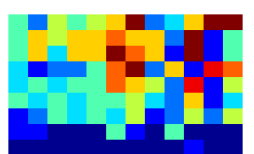

Glass ${ }^{\mathrm{G}}{ }^{\mathrm{G}}$

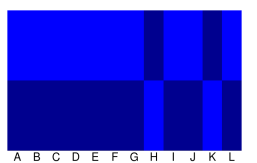

Parkinson

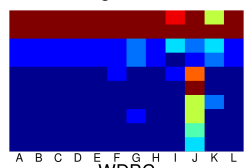

WDBC

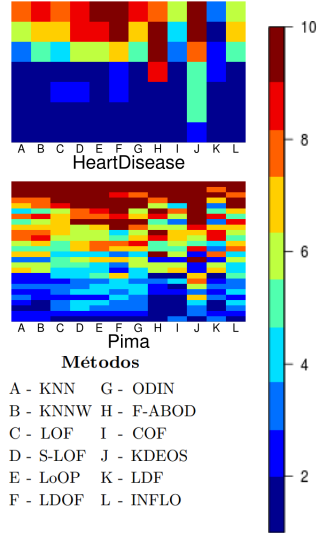

Figura 4.5: Diversidade dos rankings dos outliers em 17 bases de dados (sem duplicatas, normalizadas, aprox. 5\% outliers). As colunas estão relacionadas com os métodos nesta ordem: KNN, KNNW, LOF, SimplifiedLOF, LoOP, LDOF, ODIN, FastABOD, COF, KDEOS, LDF, INFLO. Note que FastABOD está faltando para ALOI, devido às exigências excessivas de recursos computacionais e LDF está faltando para InternetAds, devido à estimativas de densidade de kernel de altíssimas dimensões (que não levam a resultados minimamente acurados).

sobre o quão difícil cada outlier é para ser detectado (azul: fácil, vermelho: difícil), e o quão diferente essa dificuldade é para diferentes métodos.

Em poucas bases de dados, a maioria dos outliers são relativamente fáceis de detectar pela maioria dos métodos. A base Parkinson é um exemplo em que todos os métodos conseguem encontrar todos os $n$ outliers nas $2 n$ primeiras posições dos seus rankings. A base WDBC é um exemplo em que a maioria dos outliers são fáceis de detectar para a maioria dos métodos, porém dois outliers são particularmente difíceis em todos os métodos. A base Waveform é um exemplo em que menos da metade dos outliers são relativamente fáceis de detectar para vários métodos, mas pelo menos metade deles são extremamente difíceis para todos os métodos. Em geral, conseguimos verificar um amplo espectro de detectabilidade, em que alguns outliers são fáceis para todos os métodos, alguns são difíceis, e alguns possuem altos ranks para certos métodos, mas não para outros.

Com base nos resultados mencionados acima e apresentados na Figura 4.5, é possível analisar, de acordo com os verdadeiros outliers em uma base de dados, quais deles são fáceis/difíceis de detectar por cada um dos 12 métodos. Estes resultados são importantes neste trabalho, pois, através deles, é possível verificar a diversidade de detecção entre os métodos e a dificuldade de detecção dos outliers. Todas as figuras análogas àquelas na Figura 4.5 e relacionadas com todas as bases de dados coletadas estão disponíveis no repositório web mencionado no início da Seção 4.1

Os resultados apresentados na Figura 4.5 estão relacionados com bases de dados sem duplicatas e normalizados. Entretanto, o procedimento para gerar tais figuras foi aplicado em todas as bases de dados, considerando todas as versões utilizadas. Utilizamos como exemplo as bases de dados WBC e PageBlocks (considerando uma execução da versão subamostrada com aproximadamente $5 \%$ de outliers) para demonstrar os resultados obtidos quando são mantidas/removidas 


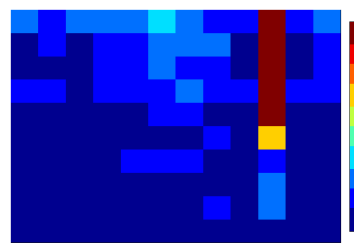

(a) WBC sem duplicatas sem normalização.

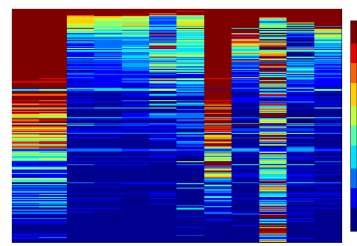

(e) PageBlocks sem duplicatas sem normalização.

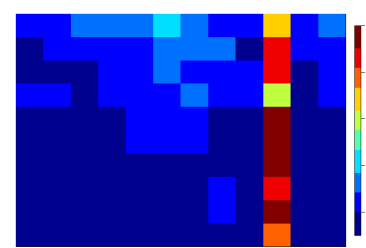

(b) WBC sem duplicatas com normalização.

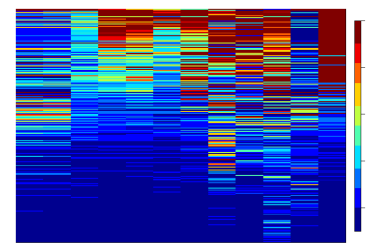

(f) PageBlocks sem duplicatas com normalização.

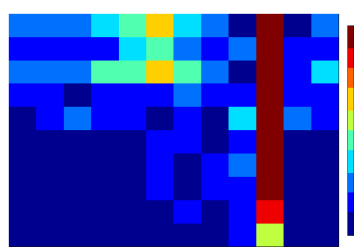

(c) WBC com duplicatas sem normalização.

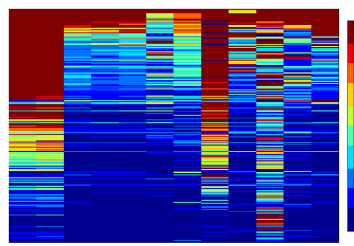

(g) PageBlocks com duplicatas sem normalização.

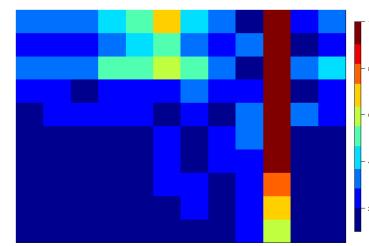

(d) WBC com duplicatas com normalização.

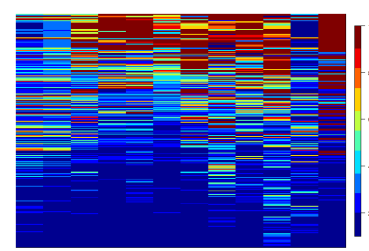

(h) PageBlocks com duplicatas com normalização.

Figura 4.6: Comparação entre a diversidade de detecção dos verdadeiros outliers entre as quatro versões utilizadas para as bases WBC e PageBlocks (aprox. 5\% outliers): com/sem duplicatas e com/sem normalização.

as duplicatas e com/sem normalização.

As Figuras 4.6(a), 4.6(b), 4.6(c) e 4.6(d) são resultados referentes às quatro versões existentes da base de dados WBC, representados por uma versão de subamostragem. Nesta base de dados, podemos afirmar que, em geral, os verdadeiros outliers se tornam um pouco mais difíceis de serem detectados quando não se remove as duplicatas. Com relação à normalização da base, poucas alterações acontecem. Geralmente, alguns outliers que foram detectados entre os $n$ primeiros perderam algumas posições no ranking de detecção das anomalias quando é aplicado normalização e, como consequência, outros outliers que não estavam bem posicionados no ranking foram ligeiramente mais fáceis de detectar.

As Figuras 4.6(e), 4.6(f), 4.6(g) $\mathrm{e} 4.6(\mathrm{~h})$ são resultados referentes às quatro versões existentes da base de dados PageBlocks (aprox. 5\% outliers), representados por uma versão de subamostragem. As mesmas conclusões relacionadas à base de dados WBC se aplicam para esta base. Contudo, é visivelmente perceptível a grande melhora na detecção dos outliers quando se aplica normalização para as técnicas de detecção KNN, KNNW e FastABOD (colunas A, B e H) para esta base de dados.

Após a análise para estas duas bases de dados e considerando todos os outros resultados que estão disponíveis no repositório on-line, podemos afirmar aqui que aplicar normalização dos dados pode, em geral, facilitar a detecção de certos outliers e dificultar a detecção de outros. Foi concluido anteriormente que os melhores resultados foram encontrados quando se aplica normalização dos dados e a remoção das duplicatas presentes. Entretanto, apesar de normalmente aplicar-se a normalização dos dados e a remoção das duplicatas (os algoritmos conseguem trabalhar melhor sem duplicatas), pode ocorrer de algum método detectar uma certa anomalia que antes não era detectada apenas em um contexto extremamente específico do problema. 
Para caracterizar as propriedades das bases de dados apresentadas na Figura 4.5 e discutir a adequação para um benchmark de detecção de outliers, definimos aqui as noções de "dificuldade" e "diversidade" de uma base de dados em respeito aos outliers. Dificuldade de uma base de dados é simplesmente definida como a média dos rankings (agrupados em 10 "bins" conforme descrito anteriormente) de todos os outliers da base de dados, reportadas por um conjunto de métodos. Diversidade caracteriza o nível de concordância dos diferentes métodos em relação aos scores dados aos outliers. Para cada outlier individual o, o score de diversidade é simplesmente definido como o desvio padrão dos ranks (agrupados em bins) obtidos para este outlier por diferentes métodos. O score de diversidade para uma base de dados então foi calculada como a raiz do valor quadrático médio (Root Mean Square ( $\underline{\mathrm{RMS}}$ ) ) dos scores de diversidade dos outliers em uma base de dados.

A Figura 4.7 mostra a posição de cada base de dados no espaço de dificuldade vs. diversidade. Para cada base de dados com variantes de subamostragem, uma elipse com $95 \%$ de confiança é exibida para indicar a extensão em que a diversidade e dificuldade podem variar com a subamostragem. As bases de dados com apenas uma variante são representados por pontos preenchidos. A figura também inclui pontos artificiais que indicam casos extremos: (1) no canto inferior esquerdo, um ponto representa o resultado "perfeito", com valor de diversidade 0 (todos os métodos concordam com os rankings agregados de todos os outliers) e valor de dificuldade 1 (todos os métodos identificam todos os $n$ outliers entre os $n$ primeiros colocados); (2) no canto superior direito, pontos representam os valores de dificuldade e diversidade esperados se todos os 12 métodos fossem substituídos por um gerador aleatório de rankings independentes ("RandomRankersIndependent"), o qual retorna rankings aleatórios uniformes de todos os objetos da base de dados; (3) no canto inferior direito, pontos representam o resultado esperado para um conjunto de 12 geradores de rankings idênticos ("RandomRankersIdentical"), resultando sempre em valores de diversidade 0 , mas variando a dificuldade por chance;

Note que a dificuldade e a diversidade não são completamente independentes um do outro. Em bases de dados que possuem baixa dificuldade, segue-se a definição de que métodos de detecção de anomalias tendem a concordar com os rankings dos outliers. Por esta razão, não há uma grande diversidade nos rankings dos outliers quando a base de dados é considerada fácil e, por isso, não esperamos encontrar bases de dados no canto superior esquerdo da Figura 4.7 . Além disto, para o modelo de geradores de rankings idênticos, se por acaso um número maior de outliers é encontrado nas primeiras posições do ranking, em que a maioria dos rankings concordam com o posicionamento dos outliers, nós podemos esperar encontrar um baixo valor de dificuldade. Enquanto geradores de rankings aleatórios individuais podem ocasionalmente, por chance, obter um alto rank para um outlier, é muito improvável para vários rankings alcançá-lo simultaneamente. Como se pode observar na Figura 4.7, isto conduz a uma variação menor nos valores de dificuldade para os 12 rankings aleatórios independentes do que para os 12 rankings aleatórios idênticos.

A partir da Figura 4.7 também podemos observar que a maioria das bases de dados consideradas neste estudo possuem valores moderados de dificuldade e diversidade. Tais bases de dados 


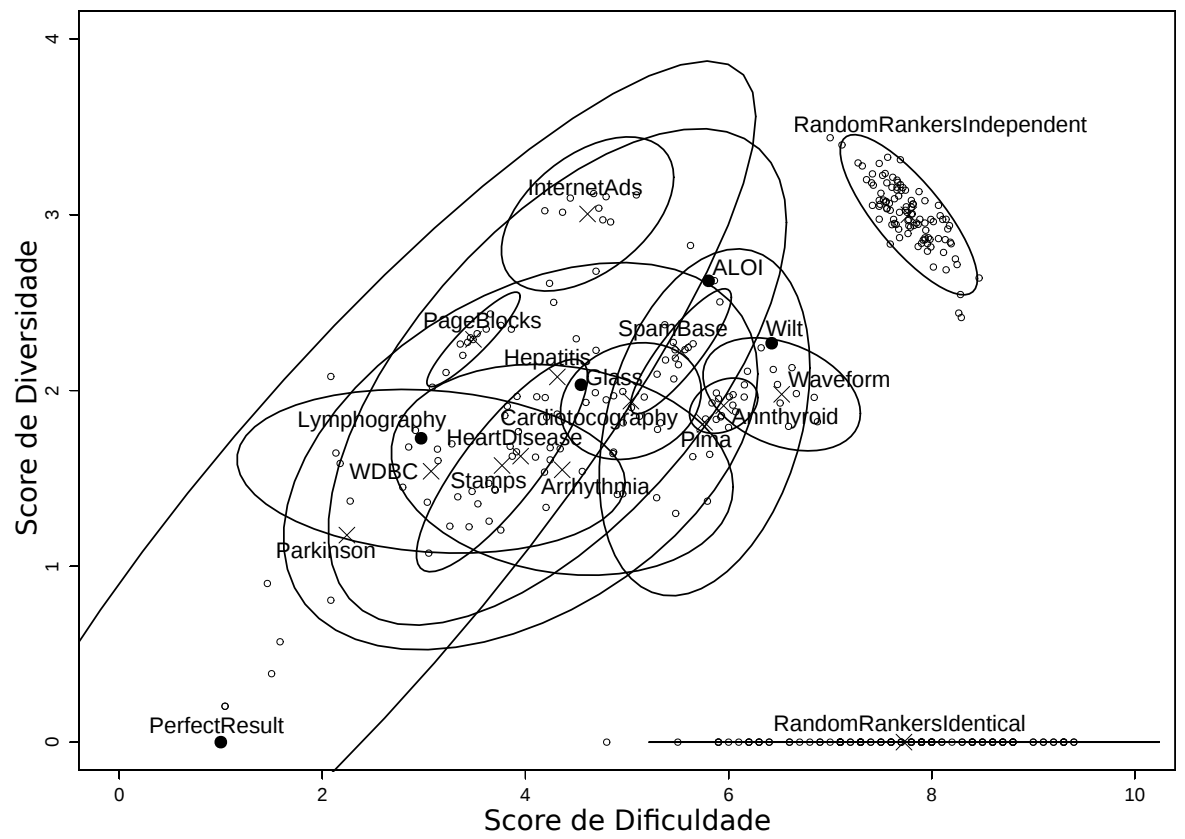

Figura 4.7: Dificuldade vs. Diversidade das bases de dados com 3\% a 5\% de outliers (sem duplicatas e normalizadas). Para cada base de dados com diferentes variantes de subamostragem, elipses com 95\% de confiança são apresentadas assim como os pontos individuais representando cada variante. As médias das elipses, que não correspondem a instâncias de dados reais, estão indicadas por um ' $x$ ' rotulada pela base de dados correspondente. Os valores dos rankings aleatórios são resultados de 100 simulações em uma base de dados com 200 instâncias, das quais 10 são outliers.

são de maior interesse nesta área de avaliação de outliers, pois eles têm variação significativa em suas características de desempenho, e permitem a possibilidade de um novo método demonstrar sua superioridade em geral sobre os métodos existentes através da detecção de outliers nestas bases de dados de forma mais consistente.

Os valores de dificuldade e diversidade observados indicam que os outliers nas bases de dados apresentadas exibem pelo menos algumas das propriedades que os métodos tentam modelar. Os 12 métodos estudados conduzem, em geral, a valores de dificuldade que são bem inferiores quando comparados com os valores apresentados pelos rankings aleatórios, indicando que uma proporção significativa das instâncias rotuladas como outliers pode, de fato, ser considerada outlier por modelos de outliers correspondentes a diferentes métodos. Para investigar se os resultados observados são significativamente diferentes daqueles apresentados por um conjunto de rankings aleatórios com a mesma dependência entre eles, foi computado o valor de dificuldade para cada base de dados baseada nos rankings alcançados pelos 12 métodos, mas após uma permutação aleatória dos rótulos das instâncias. Os resultados são mostrados na Figura 4.8 . A figura mostra para cada base de dados a distribuição dos valores de dificuldade observados como um boxplot (em azul claro) junto à um boxplot de distribuição dos valores de dificuldade obtidos após a permutação dos rótulos das instâncias (em vermelho), combinando 1.000 permutações por variante das bases de dados. Os resultados demonstram claramente que (pelo menos 


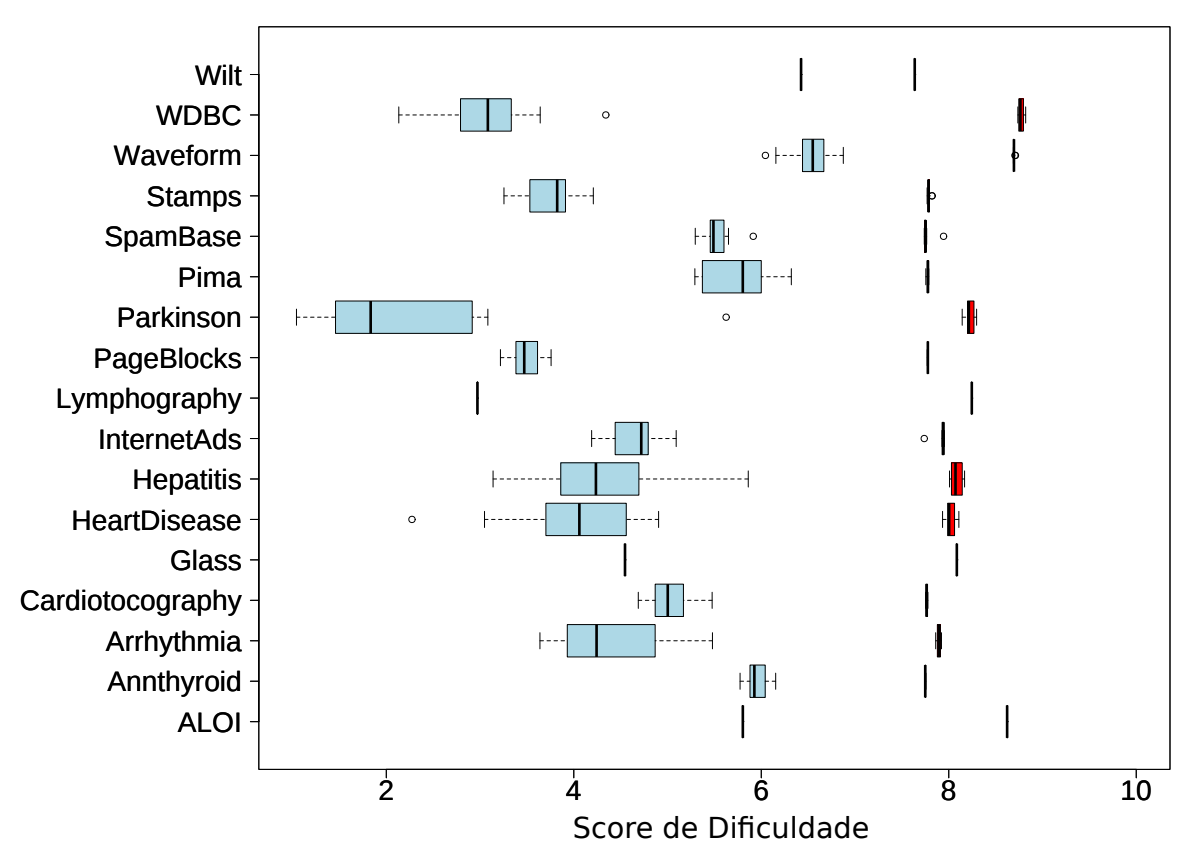

Figura 4.8: À esquerda: a distribuição dos valores observados de dificuldade para cada base de dados. À direita: para cada base de dados, a distribuição dos valores de dificuldade obtidos após permutar aleatoriamente os rótulos das instâncias. Para bases de dados com apenas uma variante entre $3 \%$ e $5 \%$ de outliers, apenas um único valor foi exibido.

parte) as instâncias rotuladas como outliers concordam com o modelo gerado para detectá-las. Para cada base de dados com 10 variantes diferentes de subamostragem, nem um único resultado aleatório em 10.000 está próximo dos valores de dificuldade observados. Para as bases de dados com apenas uma variante, nenhum dos 1.000 resultados aleatórios está próximo do valor observado.

As bases de dados que estão localizadas no centro da Figura 4.7 podem oferecer contribuições sobre os pontos fortes e fracos de diferentes métodos de detecção de outliers. No entanto, na maioria dos casos, observamos uma grande variação em ambos diversidade e dificuldade para diferentes variantes de subamostragem de uma mesma base de dados (indicadas pelas elipses). Esta variação pode ser extrema (como no caso da base Parkinson), ou mais moderada (como na base Pima). As grandes variações observadas indicam que em geral, o uso de apenas uma variante de subamostragem de uma base de dados não é apropriada quando se avalia o desempenho dos métodos de detecção de outliers.

\subsubsection{Pós-processamento}

Nesta subseção, foram realizadas alterações em algumas bases de dados conforme os resultados da detecção (ou não) dos outliers nas bases de dados, nas quais os outliers foram mais difíceis de serem detectados. Foi definido aqui que caso uma base de dados seja difícil de detectar seus outliers, técnicas de seleção de atributos seriam utilizadas. A medida selecionada para determinar a qualidade da detecção dos outliers foi a AUC. Os valores de AUC para todas as bases (considerando média e desvio padrão para as bases com mais de uma versão de subamos- 
tragem) estão disponíveis no Apêndice A deste documento. Os valores de AUC no Apêndice A estão relacionados com a média entre os melhores valores para cada método (seleção do parâmetro $k$ que possui o maior valor de AUC).

Todas as bases (exceto versões subamostradas) foram verificadas e, caso em alguma de suas versões (com/sem duplicatas e com/sem normalização) o resultado de AUC foi abaixo de 0.7, dois métodos de seleção de atributos foram aplicados com o intuito de melhorar a detecção dos outliers (aumentar os valores de AUC). Ambos os métodos consistem na utilização de árvores de decisão para selecionar atributos relevantes à discriminação entre outliers e inliers. As árvores de decisão são ferramentas poderosas para detectar atributos relevantes na classificação. Espera-se que com a utilização dessas seja possível reduzir o espaço de atributos para um subespaço menor, no qual os outliers sejam mais fáceis de serem detectados. Ambos os métodos visam lidar com a natureza desbalanceada das classes (outliers e inliers).

O primeiro método consiste na utilização de uma árvore de decisão juntamente com uma matriz de custo. Tal matriz dá um peso maior ao errar a classificação de instâncias que pertencem a uma classe minoritária do que errar instâncias de uma classe majoritária. Esse peso foi alcançado ao dividir a quantidade de inliers presentes nas bases pela quantidade de outliers. Desta forma, se o classificador errar todos os outliers eles terão o mesmo peso de errar todos os inliers. O objetivo do classificador é minimizar este custo e, consequentemente, acertar mais outliers através de um conjunto menor de atributos. A árvore de decisão utilizada foi a J48 (Quinlan, 1993) assim como ela está implementada no WEKA (Hall et al., 2009). Tal abordagem foi realizada utilizando cross-validation com 10 folds.

O segundo método busca reduzir o espaço de atributos utilizando uma árvore de decisão sem poda. Ao construir toda a árvore, a qual possivelmente classifica corretamente todas as instâncias da base (inliers e outliers), os atributos necessários para discriminar ambas as classes serão descritos pelos caminhos percorridos pela árvore. A árvore de decisão utilizada aqui também foi a J48 (Quinlan, 1993) assim como ela está implementada no WEKA (Hall et al. 2009).

Ambos os métodos de seleção de atributos selecionaram todos os atributos presentes na base Cardiotocography, o que torna os resultados para tal base irrelevantes. Portanto, a base Cardiotocography foi removida dos resultados apresentados nesta seção.

As bases de dados selecionadas sem duplicatas e sem normalização (referente à Tabela A.1 presente no Apêndice A seguindo o limiar de AUC descrito anteriormente foram: Cardiotocography_22, HeartDisease_44, Hepatitis_16 e Pima_35. Os resultados estão presentes na Figura 4.9. Ambos os métodos de seleção de atributos obtiveram o mesmo resultado para a base Pima_35, selecionando os mesmos atributos para as duas árvores de decisão e, com isso, os resultados para ambas as técnicas foram idênticos. Para a base HeartDisease_44 a técnica de seleção de atributos $\mathrm{J} 48$ sem poda considerou todos os atributos originais em seu caminho para diferenciar outliers de inliers, obtendo o mesmo resultado original. O grande diferencial apresentado nesta parte dos resultados foi ao aplicar estas técnicas na base Hepatitis_16. Tal base que apenas tinha seu valor médio de AUC em 0.6280, obteve uma melhora considerável 


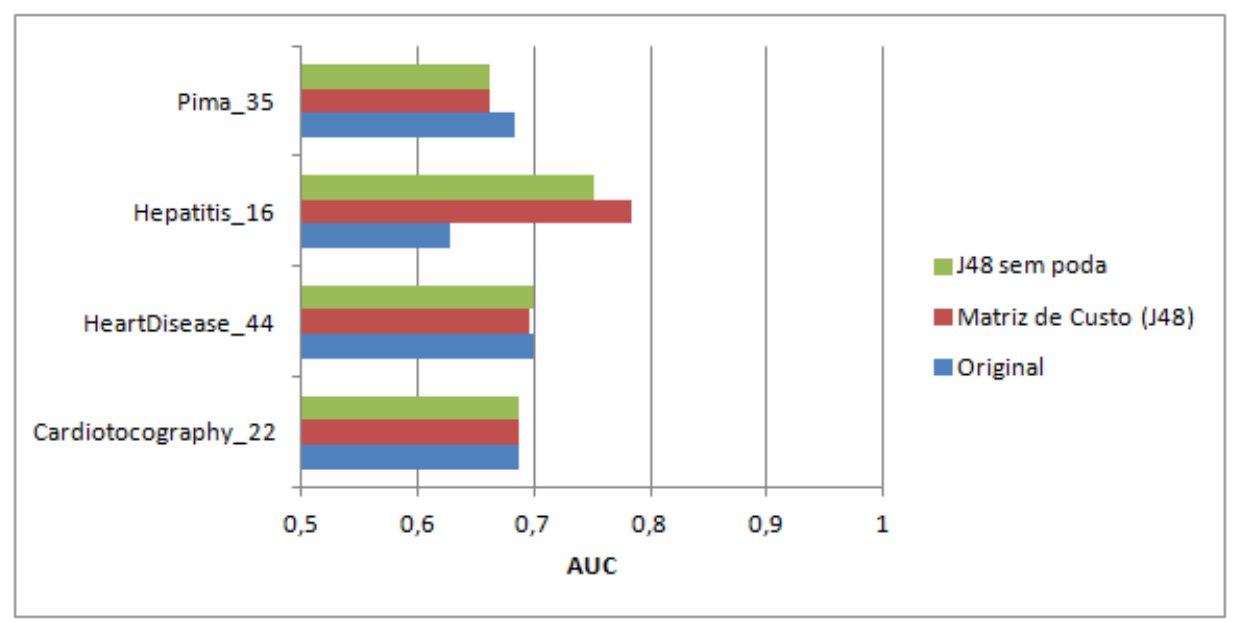

Figura 4.9: Novos valores de AUClaplicando técnicas de seleção de atributos em bases de dados sem duplicatas e sem normalização.

em ambas as técnicas de seleção de atributos (0.7836 utilizando matriz de custo e 0.7520 utilizando J48 sem poda). Entretanto, pode haver casos em que os outliers fiquem mais difíceis de serem detectados neste novo subespaço, como ocorre na base Pima_35. No conjunto original de atributos, o valor de AUC era 0.6840. Ao aplicar as técnicas, o valor de AUC caiu para 0.6615.

Além do ótimo resultado encontrado para a base Hepatitis_16, seu conjunto de atributos foi reduzido de 19 para apenas 3 atributos na técnica utilizando matriz de custo e 10 na técnica J48 sem poda. Para a base Pima_35, o atributo "att4" foi removido em ambas as técnicas, passando de 8 para 7 atributos. A base HeartDisease_44 também teve apenas um atributo removido na técnica utilizando matriz de custo, passando de 13 para 12 atributos.

As bases de dados selecionadas sem duplicatas e com normalização (referente à Tabela A.2 presente no Apêndice A) foram: Annthyroid_07, Cardiotocography_22, Parkinson_75, SpamBase_40 e WPBC. Os resultados estão presentes na Figura 4.10. Para as bases WPBC e Parkinson_75, ambos os métodos de seleção de atributos obtiveram o mesmo resultado original, selecionando todos os atributos originais para compor a árvore de decisão. Conforme a Figura 4.10, todos os resultados das técnicas de seleção de atributos foram iguais e/ou superiores aos resultados originais de cada base. Considerando estes resultados, é importante destacar o grande salto apresentado para a base Annthyroid_07 na Figura 4.10. Seu resultado original de AUC era 0.6921 e, aplicando as técnicas de seleção de atributos, os resultados foram de 0.7528 utilizando matriz de custo e 0.7513 utilizando J48 sem poda. Além desta melhora, esta base ainda teve vários atributos removidos, passando de 21 atributos para 7 utilizando matriz de custo e 10 utilizando J48 sem poda. Já é perceptível a melhora dos resultados (facilitando bases que antes eram difíceis) aplicando estas técnicas.

Considerando bases de dados com duplicatas e sem normalização (referente à Tabela A.3 presente no Apêndice A, apenas a base Cardiotocography_22 teve seu valor de AUC abaixo de 0.7. Entretanto, conforme mencionado anteriormente, as técnicas de seleção de atributos mantêm em suas árvores todos os atributos originais desta base. Devido a isto, nenhum resultado de pós-processamento foi apresentado para esta versão (com duplicatas e sem normalização). 


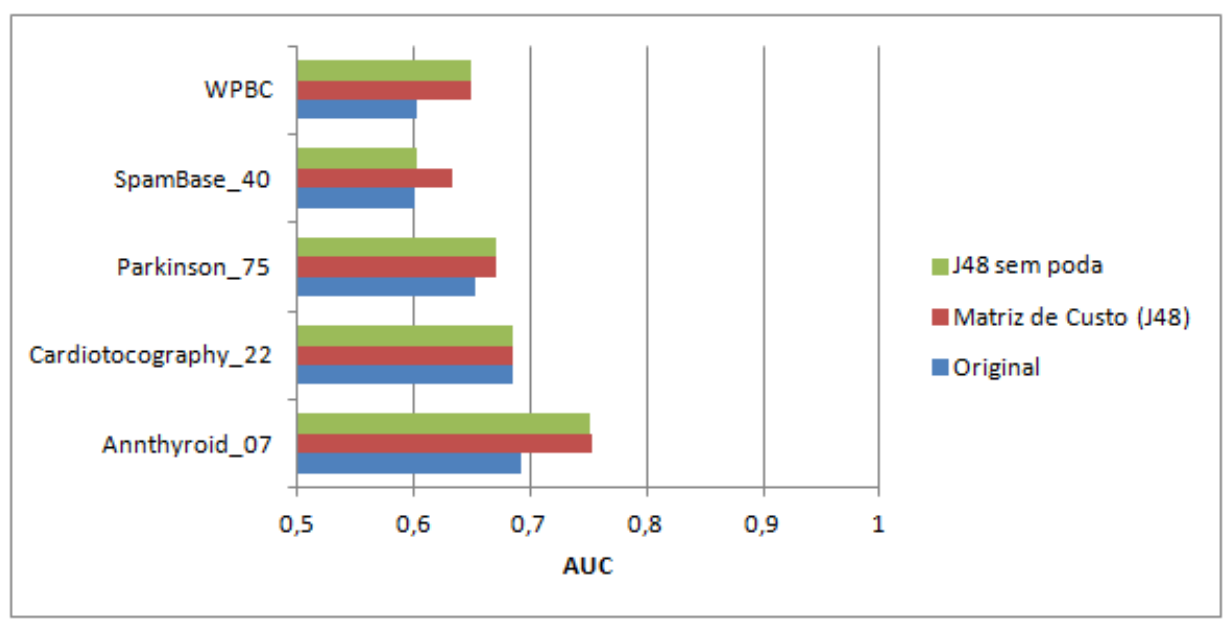

Figura 4.10: Novos valores de $\mathrm{AUC}$ aplicando técnicas de seleção de atributos em bases de dados sem duplicatas e com normalização.

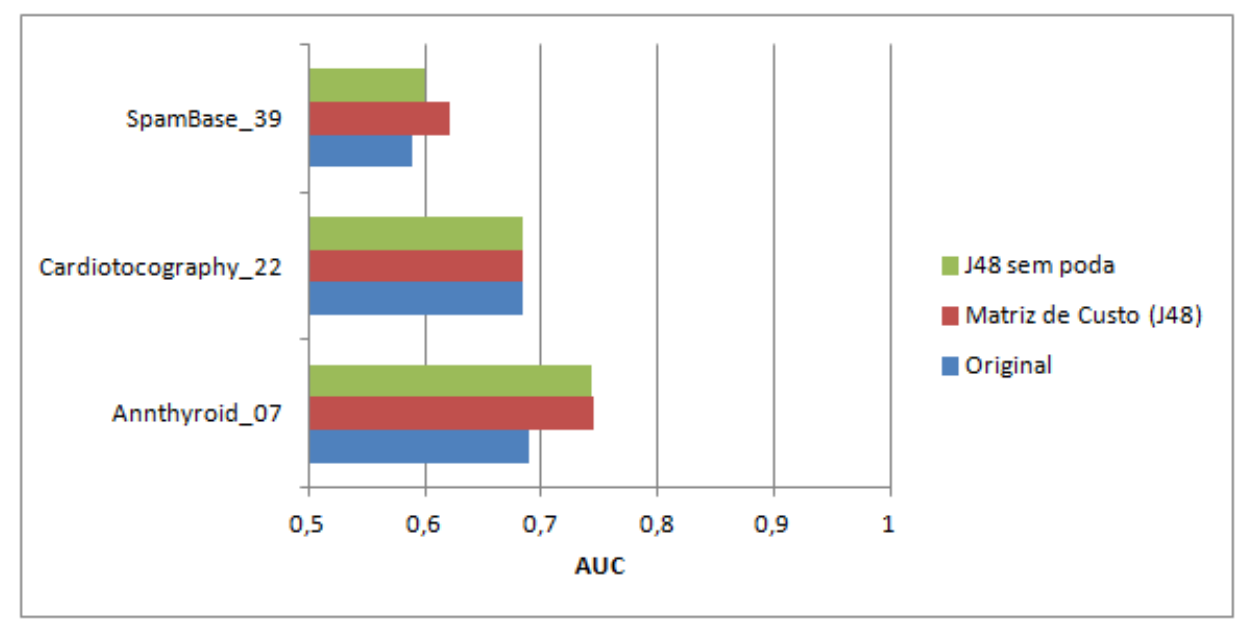

Figura 4.11: Novos valores de $\mathrm{AUC}$ aplicando técnicas de seleção de atributos em bases de dados com duplicatas e com normalização.

As bases de dados selecionadas com duplicatas e com normalização (referente à Tabela A.4 presente no Apêndice A foram: Annthyroid_07, Cardiotocography_22 e SpamBase_39. Os resultados estão presentes na Figura 4.11. A base Annthyroid_07 passou de 21 atributos para 7 utilizando matriz de custo e 10 utilizando J48 sem poda. Já a base SpamBase_39 passou de 57 atributos para 46 tilizando matriz de custo e 52 utilizando J48 sem poda. Para estes resultados é importante destacar a melhora nos resultados da base Annthyroid_07 na Figura 4.11. O resultado original de AUC que antes era de 0.6898 passou para 0.7449 e 0.7439 aplicando as técnicas de matriz de custo e $\mathrm{J} 48$ sem poda respectivamente. Os resultados aplicando ambas as técnicas de seleção de atributos foram superiores aos resultados originais para as bases Annthyroid_07 e SpamBase_39.

De acordo com os resultados nesta seção, entre as duas técnicas de seleção de atributos, a matriz de custo utilizando a árvore de decisão J48 obteve melhores resultados. Considerando todos os resultados apresentados nesta seção, podemos afirmar que utilizar técnicas de seleção de atributos quando se conhece o ground truth das instâncias facilita a detecção de parte das 
anomalias, o que sugere a importância da ainda pouco explorada área de algoritmos de detecção de outliers em subespaços dos atributos (Kriegel et al., 2009b; Nguyen et al., 2010; Müller et al., 2011, 2012; Kriegel et al., 2012; Keller et al., 2012). 


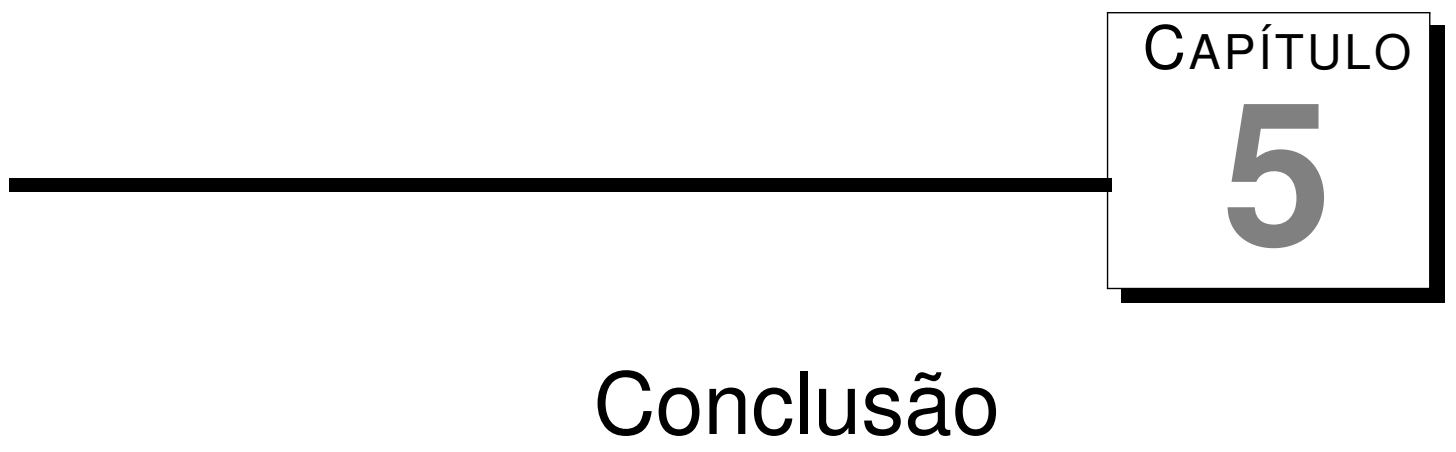

Nesse trabalho de criação e análise de um conjunto benchmark de bases de dados para detecção de outliers e comparação de vários métodos de detecção, atacamos uma área pouco explorada ainda em detecção de anomalias. Contribuir com um conjunto de bases de dados padronizados e disponíveis para qualquer pessoa utilizar e fazer análises é um avanço para esta área. Não apenas isto, mas analisar vários métodos de detecção de outliers, cada qual utilizando diferentes critérios de detecção, considerando uma grande coleção de bases de dados era algo necessário na literatura. A partir deste trabalho, podemos analisar a capacidade de detecção de cada método e a validade de um grande conjunto de bases de dados para compor um benchmark padronizado e aberto para a comunidade.

Pela disponibilização do benchmark de bases de dados para detecção de outliers, sempre que for necessária a comparação entre algoritmos existentes e novos algoritmos para detecção de outliers, o benchmark poderá ser utilizado publicamente. A nossa metodologia de criação de um benchmark de bases de dados para detecção de outliers respeitou os três primeiros requisitos citados por Emmott et al. (2013) (vide Seção 3.2). O último requisito não foi abordado neste trabalho devido à enorme complexidade de trabalhar com variações sistemáticas dos aspectos das bases de dados com relação à subjetividade do conceito de outlier.

Cada base de dados selecionada para compor o benchmark foi analisada cuidadosamente e, ou por ter sido utilizada na literatura ou pela semântica clara diferenciando outliers de inliers, foi incluída no benchmark. Através deste benchmark foi possível comparar o desempenho de vários algoritmos (clássicos e novos) de detecção de outliers com 100 diferentes valores do parâmetro de vizinhança $(k)$. Todos os resultados (para cada algoritmo, parâmetro e base de dados) estão disponibilizados publicamente em nosso repositório on-line (http:// lapad-web.icmc . usp.br/repositories/outlier-evaluation/), de forma que todos esses experimentos não precisarão ser re-executados em futuros estudos que envolvem a comparação com os algoritmos em questão. Como resultado mais relevante observado, dentre todos os algorit- 
mos estudados aqui, os métodos clássicos KNN, KNNW e LOF ainda superam em média os métodos mais recentes. Ou seja, nenhum dos métodos mais modernos de detecção de outliers ofereceu uma melhoria significativa para o estado da arte.

Após os resultados finais e a aplicação das técnicas de seleção de atributos em bases que são relativamente difíceis de detectar os outliers (considerando os 12 métodos de detecção utilizados), foi perceptível a melhora na detecção das anomalias quando se utiliza atributos relevantes para distinguir outliers de inliers, o que sugere a importância de se avançar o estado da arte na área de detecção de outliers em subespaços de atributos, que ainda é pouco explorada.

Outra área que também obteve avanços com este trabalho foi a área de medidas para avaliação de algoritmos de detecção de anomalias. Três métodos principais foram discutidos e utilizados $(\mathrm{AUC}, P @ n$ e $\mathrm{AP}$ e dois deles tiveram suas versões ajustadas $(P @ n$ e $\mathrm{AP})$, com apoio e contribuição intelectual dos professores mencionados no resumo deste trabalho.

Futuras extensões deste trabalho podem incorporar áreas como a comparação entre algoritmos de ensemble para detecção de outliers, analisando diferentes maneiras de induzir diversidade nos membros do ensemble e diferentes formas de combinar os scores/rankings finais. Outra possível extensão é analisar métodos especiais de detecção de outliers em bases de dados com alta dimensionalidade, como os métodos de detecção em subespaços. Ter em mãos este benchmark possibilita a utilização dos resultados disponíveis neste trabalho para qualquer outra finalidade que necessite dos resultados dos algoritmos apresentados aqui. 


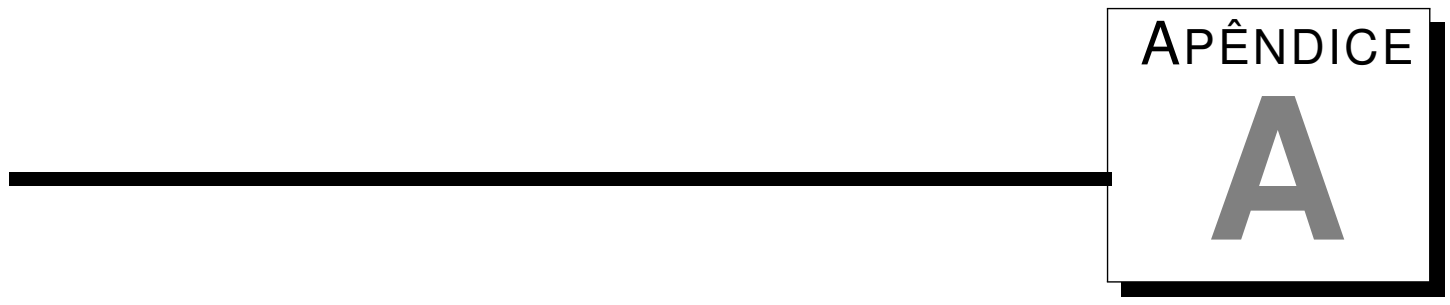

\section{Resultados AUC}

As tabelas deste apêndice contém os resultados da medida de avaliação AUC para todas as bases de dados deste projeto. As bases de dados foram representadas com os maiores valores de AUC dentre todos os valores do parâmetro $k$ para cada método e, assim, foi extraída a média dos valores de AUC. O nome das bases de dados com semântica possui uma numeração à frente relacionado com a porcentagem de outliers presentes na respectiva base. Os resultado para bases de dados com mais de uma execução estão representados pela fórmula: média \pm desvio padrão. 
Tabela A.1: Resultado da medida de avaliação AUC para bases de dados sem duplicatas e sem normalização.

\begin{tabular}{|c|c|}
\hline Bases - Semântica & AUC \\
\hline Annthyroid_02 & $0.7896 \pm 0.0087$ \\
\hline Annthyroid_05 & $0.7515 \pm 0.0078$ \\
\hline Annthyroid_07 & 0.7245 \\
\hline Arrhythmia_02 & $0.8514 \pm 0.1055$ \\
\hline Arrhythmia_05 & $0.8346 \pm 0.0321$ \\
\hline Arrhythmia_10 & $0.8306 \pm 0.0160$ \\
\hline Arrhythmia_20 & $0.7840 \pm 0.0345$ \\
\hline Arrhythmia_46 & 0.7675 \\
\hline Cardiotocography_02 & $0.8556 \pm 0.0277$ \\
\hline Cardiotocography_05 & $0.8101 \pm 0.0158$ \\
\hline Cardiotocography_10 & $0.7681 \pm 0.0134$ \\
\hline Cardiotocography_20 & $0.6997 \pm 0.0048$ \\
\hline Cardiotocography_22 & 0.6867 \\
\hline HeartDisease_02 & $0.8523 \pm 0.1098$ \\
\hline HeartDisease_05 & $0.8411 \pm 0.0522$ \\
\hline HeartDisease_10 & $0.7648 \pm 0.0446$ \\
\hline HeartDisease_20 & $0.7389 \pm 0.0304$ \\
\hline HeartDisease_44 & 0.6994 \\
\hline Hepatitis_05 & $0.7649 \pm 0.0812$ \\
\hline Hepatitis_10 & $0.7085 \pm 0.0613$ \\
\hline Hepatitis_16 & 0.6280 \\
\hline PageBlocks_02 & $0.9502 \pm 0.0076$ \\
\hline PageBlocks_05 & $0.9286 \pm 0.0056$ \\
\hline PageBlocks_09 & 0.9037 \\
\hline Parkinson_05 & $0.9541 \pm 0.0423$ \\
\hline Parkinson_10 & $0.8729 \pm 0.0441$ \\
\hline Parkinson_20 & $0.8124 \pm 0.0404$ \\
\hline Parkinson_75 & 0.7380 \\
\hline Pima_02 & $0.7905 \pm 0.0348$ \\
\hline Pima_05 & $0.7376 \pm 0.0366$ \\
\hline Pima_10 & $0.7261 \pm 0.0228$ \\
\hline Pima_20 & $0.7196 \pm 0.0129$ \\
\hline Pima_35 & 0.6840 \\
\hline SpamBase_02 & $0.7656 \pm 0.0352$ \\
\hline SpamBase_05 & $0.7644 \pm 0.0093$ \\
\hline SpamBase_10 & $0.7502 \pm 0.0148$ \\
\hline SpamBase_20 & $0.7421 \pm 0.0075$ \\
\hline SpamBase_40 & 0.7396 \\
\hline Stamps_02 & $0.9559 \pm 0.0137$ \\
\hline Stamps_05 & $0.9292 \pm 0.0118$ \\
\hline Stamps_09 & 0.9281 \\
\hline Wilt_02 & $0.8804 \pm 0.0091$ \\
\hline Wilt_05 & 0.8142 \\
\hline
\end{tabular}

\begin{tabular}{|l|c|}
\hline Bases - Literatura & AUC \\
\hline ALOI & 0.7948 \\
\hline \hline KDDCup99_1ofn & 0.7948 \\
\hline KDDCup99_catremoved & 0.8105 \\
\hline KDDCup99_idf & 0.8105 \\
\hline \hline Lymphography_1ofn & 0.9906 \\
\hline Lymphography_catremoved & 0.9595 \\
\hline Lymphography_idf & 0.9595 \\
\hline \hline Shuttle & $0.9713 \pm 0.0056$ \\
\hline \hline Waveform & $0.7878 \pm 0.0139$ \\
\hline \hline WBC & $0.9930 \pm 0.0076$ \\
\hline \hline WDBC & $0.9935 \pm 0.0048$ \\
\hline
\end{tabular}


Tabela A.2: Resultado da medida de avaliação AUC para bases de dados sem duplicatas e com normalização.

\begin{tabular}{|c|c|}
\hline Bases - Semântica & AUC \\
\hline Annthyroid_02 & $0.7705 \pm 0.0141$ \\
\hline Annthyroid_05 & $0.7192 \pm 0.0088$ \\
\hline Annthyroid_07 & 0.6921 \\
\hline Arrhythmia_02 & $0.9010 \pm 0.0494$ \\
\hline Arrhythmia_05 & $0.8469 \pm 0.0408$ \\
\hline Arrhythmia_10 & $0.8177 \pm 0.0232$ \\
\hline Arrhythmia_20 & $0.7723 \pm 0.0327$ \\
\hline Arrhythmia_46 & 0.7520 \\
\hline Cardiotocography_02 & $0.8539 \pm 0.0317$ \\
\hline Cardiotocography_05 & $0.7937 \pm 0.0235$ \\
\hline Cardiotocography_10 & $0.7489 \pm 0.0130$ \\
\hline Cardiotocography_20 & $0.6984 \pm 0.0073$ \\
\hline Cardiotocography_22 & 0.6845 \\
\hline HeartDisease_02 & $0.9193 \pm 0.0682$ \\
\hline HeartDisease_05 & $0.9228 \pm 0.0452$ \\
\hline HeartDisease_10 & $0.8984 \pm 0.0307$ \\
\hline HeartDisease_20 & $0.8421 \pm 0.0300$ \\
\hline HeartDisease_44 & 0.7762 \\
\hline Hepatitis_05 & $0.9266 \pm 0.0205$ \\
\hline Hepatitis_10 & $0.8561 \pm 0.0226$ \\
\hline Hepatitis_16 & 0.8358 \\
\hline InternetAds_02 & $0.9122 \pm 0.0266$ \\
\hline InternetAds_05 & $0.8484 \pm 0.0234$ \\
\hline InternetAds_10 & $0.8130 \pm 0.0188$ \\
\hline InternetAds_19 & 0.7426 \\
\hline PageBlocks_02 & $0.9608 \pm 0.0036$ \\
\hline PageBlocks_05 & $0.9179 \pm 0.0071$ \\
\hline PageBlocks_09 & 0.8839 \\
\hline Parkinson_05 & $0.9833 \pm 0.0299$ \\
\hline Parkinson_10 & $0.9637 \pm 0.0219$ \\
\hline Parkinson_20 & $0.9344 \pm 0.0192$ \\
\hline Parkinson_75 & 0.6523 \\
\hline Pima_02 & $0.7912 \pm 0.0369$ \\
\hline Pima_05 & $0.7764 \pm 0.0189$ \\
\hline Pima_10 & $0.7693 \pm 0.0268$ \\
\hline Pima_20 & $0.7649 \pm 0.0141$ \\
\hline Pima_35 & 0.7616 \\
\hline SpamBase_02 & $0.8082 \pm 0.0191$ \\
\hline SpamBase_05 & $0.7678 \pm 0.0142$ \\
\hline SpamBase_10 & $0.7177 \pm 0.0170$ \\
\hline SpamBase_20 & $0.6620 \pm 0.0120$ \\
\hline SpamBase_40 & 0.6002 \\
\hline Stamps_02 & $0.9520 \pm 0.0174$ \\
\hline Stamps_05 & $0.9212 \pm 0.0072$ \\
\hline Stamps_09 & 0.9117 \\
\hline Wilt_02 & $0.7819 \pm 0.0096$ \\
\hline Wilt_05 & 0.7122 \\
\hline
\end{tabular}

\begin{tabular}{|l|c|}
\hline Bases - Literatura & AUC \\
\hline ALOI & 0.8080 \\
\hline \hline Glass & 0.8948 \\
\hline \hline Ionosphere & 0.9603 \\
\hline \hline KDDCup99_1ofn & 0.9904 \\
\hline KDDCup99_catremoved & 0.9897 \\
\hline KDDCup99_idf & 0.9897 \\
\hline \hline Lymphography_1ofn & 1.0000 \\
\hline Lymphography_catremoved & 0.9372 \\
\hline Lymphography_idf & 0.9372 \\
\hline \hline PenDigits & $0.9906 \pm 0.0021$ \\
\hline \hline Shuttle & $0.9571 \pm 0.0452$ \\
\hline \hline Waveform & $0.7702 \pm 0.0130$ \\
\hline \hline WBC & $0.9928 \pm 0.0074$ \\
\hline \hline WDBC & $0.9592 \pm 0.0223$ \\
\hline \hline WPBC & 0.6018 \\
\hline
\end{tabular}


Tabela A.3: Resultado da medida de avaliação AUC para bases de dados com duplicatas e sem normalização.

\begin{tabular}{|l|c|}
\hline Bases - Semântica & AUC \\
\hline Annthyroid_02 & $0.7906 \pm 0.0106$ \\
\hline Annthyroid_05 & $0.7517 \pm 0.0078$ \\
\hline Annthyroid_07 & 0.7240 \\
\hline \hline Cardiotocography_02 & $0.8522 \pm 0.0205$ \\
\hline Cardiotocography_05 & $0.8028 \pm 0.0085$ \\
\hline Cardiotocography_10 & $0.7736 \pm 0.0139$ \\
\hline Cardiotocography_20 & $0.6930 \pm 0.0052$ \\
\hline Cardiotocography_22 & 0.6845 \\
\hline \hline PageBlocks_02 & $0.9417 \pm 0.0116$ \\
\hline PageBlocks_05 & $0.9021 \pm 0.0103$ \\
\hline PageBlocks_09 & 0.8653 \\
\hline \hline SpamBase_02 & $0.8104 \pm 0.0202$ \\
\hline SpamBase_05 & $0.7777 \pm 0.0220$ \\
\hline SpamBase_10 & $0.7689 \pm 0.0084$ \\
\hline SpamBase_20 & $0.7597 \pm 0.0059$ \\
\hline SpamBase_40 & 0.7563 \\
\hline \hline Wilt_02 & $0.8839 \pm 0.0097$ \\
\hline Wilt_05 & 0.8097 \\
\hline
\end{tabular}

\begin{tabular}{|l|c|}
\hline Bases - Literatura & AUC \\
\hline ALOI & 0.7962 \\
\hline \hline KDDCup99_1ofn & 0.7127 \\
\hline KDDCup99_catremoved & 0.7126 \\
\hline KDDCup99_idf & 0.7144 \\
\hline \hline WBC & $0.9963 \pm 0.0034$ \\
\hline
\end{tabular}

Tabela A.4: Resultado da medida de avaliação AUC para bases de dados com duplicatas e com normalização.

\begin{tabular}{|l|c|}
\hline Bases - Semântica & AUC \\
\hline Annthyroid_02 & $0.7724 \pm 0.0270$ \\
\hline Annthyroid_05 & $0.7201 \pm 0.0095$ \\
\hline Annthyroid_07 & 0.6898 \\
\hline \hline Cardiotocography_02 & $0.8318 \pm 0.0299$ \\
\hline Cardiotocography_05 & $0.7824 \pm 0.0126$ \\
\hline Cardiotocography_10 & $0.7558 \pm 0.0155$ \\
\hline Cardiotocography_20 & $0.6952 \pm 0.0095$ \\
\hline Cardiotocography_22 & 0.6845 \\
\hline \hline PageBlocks_02 & $0.9536 \pm 0.0040$ \\
\hline PageBlocks_05 & $0.9107 \pm 0.0104$ \\
\hline PageBlocks_09 & 0.8376 \\
\hline \hline SpamBase_02 & $0.8333 \pm 0.0305$ \\
\hline SpamBase_05 & $0.7695 \pm 0.0180$ \\
\hline SpamBase_10 & $0.7265 \pm 0.0203$ \\
\hline SpamBase_20 & $0.6617 \pm 0.0115$ \\
\hline SpamBase_39 & 0.5891 \\
\hline \hline Wilt_02 & $0.7825 \pm 0.0199$ \\
\hline Wilt_05 & 0.7092 \\
\hline
\end{tabular}

\begin{tabular}{|l|c|}
\hline Bases - Literatura & AUC \\
\hline ALOI & 0.7962 \\
\hline \hline KDDCup99_1ofn & 0.9897 \\
\hline KDDCup99_catremoved & 0.9831 \\
\hline KDDCup99_idf & 0.9904 \\
\hline \hline WBC & $0.9963 \pm 0.0033$ \\
\hline
\end{tabular}




\section{Referências Bibliográficas}

Abe, N., Zadrozny, B., and Langford, J. (2006). Outlier detection by active learning. In Proceedings of the 12th ACM SIGKDD International Conference on Knowledge Discovery and Data Mining, KDD '06, pages 504-509, New York, NY, USA. ACM.

Achtert, E., Kriegel, H.-P., Schubert, E., and Zimek, A. (2013). Interactive data mining with 3dparallel-coordinate-trees. In Ross, K. A., Srivastava, D., and Papadias, D., editors, SIGMOD Conference, pages 1009-1012. ACM.

Angiulli, F. and Pizzuti, C. (2002). Fast outlier detection in high dimensional spaces. pages 15-26, Helsinki, Finland.

Barnett, V. and Lewis, T. (1994). Outliers in Statistical Data. John Wiley \& Sons, 3rd edition.

Bigus, J. P. (1996). Data Mining with Neural Networks: Solving Business Problems from Application Development to Decision Support. McGraw-Hill.

Breunig, M. M., Kriegel, H.-P., Ng, R., and Sander, J. (2000). Lof: Identifying density-based local outliers. pages 93-104, Dallas, TX.

Campos, G. O., Zimek, A., Sander, J., Campello, R. J. G. B., Micenková, B., Schubert, E., Assent, I., and Houle, M. E. (2015). On the evaluation of unsupervised outlier detection methods. Proceedings of European Conference on Machine Learning and Principles and Practice of Knowledge Discovery in Databases (ECML PKDD).

Chandola, V., Banerjee, A., and Kumar, V. (2012). Anomaly detection for discrete sequences: A survey. IEEE Transactions on Knowledge and Data Engineering, pages 823-839.

Craswell, N. (2009a). Precision at n. In Encyclopedia of Database Systems, pages 2127-2128.

Craswell, N. (2009b). R-precision. In Encyclopedia of Database Systems, page 2453.

Davis, J. and Goadrich, M. (2006). The relationship between Precision-Recall and ROC curves. pages 233-240. 
de Vries, T., Chawla, S., and Houle, M. E. (2012). Density-preserving projections for largescale local anomaly detection. Knowl. Inf. Syst., 32(1):25-52.

Emmott, A. F., Das, S., Dietterich, T., Fern, A., and Wong, W.-K. (2013). Systematic construction of anomaly detection benchmarks from real data. pages 16-21, Chicago, IL.

Fayyad, U. M., Shapiro, G. P., Smyth, P., and Uthurusamy, R. (1996). Advances in Knowledge Discovery and Data Mining. MIT Press.

Frank, A. and Asuncion, A. (2010). UCI machine learning repository.

Gan, G., Ma, C., and Wu, J. (2007). Data Clustering: Theory, Algorithms, and Applications. Society for Industrial and Applied Mathematics (SIAM), illustrated edition edition.

Gao, J. and Tan, P.-N. (2006). Converting output scores from outlier detection algorithms into probability estimates. pages 212-221, Hong Kong, China.

Hall, M., Frank, E., Holmes, G., Pfahringer, B., Reutemann, P., and Witten, I. H. (2009). The weka data mining software: An update. SIGKDD Explor. Newsl., 11(1):10-18.

Han, J. and Kamber, M. (2006). Data Mining: Concepts and Techniques. Morgan Kaufmann, 2nd edition.

Han, J., Kamber, M., and Pei, J. (2011). Data Mining: Concepts and Techniques. Morgan Kaufmann Publishers Inc., San Francisco, CA, USA, 3rd edition.

Hautamaki, V., Karkkainen, I., and Franti, P. (2004). Outlier detection using k-nearest neighbour graph. In Proceedings of the Pattern Recognition, 17th International Conference on (ICPR'04) Volume 3 - Volume 03, ICPR '04, pages 430-433, Washington, DC, USA. IEEE Computer Society.

Hawkins, D. (1980). Identification of Outliers. Chapman and Hall.

He, Z., Deng, S., and Xu, X. (2005). A unified subspace outlier ensemble framework for outlier detection. In Fan, W., Wu, Z., and Yang, J., editors, Advances in Web-Age Information Management, volume 3739 of Lecture Notes in Computer Science, pages 632-637. Springer Berlin Heidelberg.

Howell, D. C. (1998). Statistical Methods in Human Sciences. New York: Wadsworth.

Hubert, L. and Arabie, P. (1985). Comparing partitions. 2(1):193-218.

Jain, A. K. and Dubes, R. C. (1988). Prenticed Hall, Englewood Cliffs. Algorithms for Clustering Data.

Jin, W., Tung, A. K. H., Han, J., and Wang, W. (2006). Ranking outliers using symetric neighborhood relationship. pages 577-593, Singapore. 
Keller, F., Müller, E., and Böhm, K. (2012). Hics: high contrast subspaces for density-based outlier ranking. Washington, DC.

Knorr, E. M. and Ng, R. T. (1998). Algorithms for mining distance-based outliers in large datasets. pages 392-403, New York, NY.

Knorr, E. M., Ng, R. T., and Tucanov, V. (2000). Distance-based outliers: Algorithms and applications. The VLDB Journal, pages 8(3-4):237-253.

Kriegel, H., Kroger, P., Schubert, E., and Zimek, A. (2012). Outlier detection in arbitrarily oriented subspaces. In Data Mining (ICDM), 2012 IEEE 12th International Conference on, pages $379-388$.

Kriegel, H.-P., Kröger, P., Schubert, E., and Zimek, A. (2009a). Loop: local outlier probabilities. pages 1649-1652, Hong Kong, China.

Kriegel, H.-P., Kröger, P., Schubert, E., and Zimek, A. (2009b). Outlier detection in axis-parallel subspaces of high dimensional data. pages 831-838, Bangkok, Thailand.

Kriegel, H.-P., Kröger, P., Schubert, E., and Zimek, A. (2011). Interpreting and unifying outlier scores. pages 13-24, Mesa, AZ.

Kriegel, H.-P., Schubert, M., and Zimek, A. (2008). Angle-based outlier detection in highdimensional data. pages 444-452, Las Vegas, NV.

Latecki, L., Lazarevic, A., and Pokrajac, D. (2007). Outlier detection with kernel density functions. In Perner, P., editor, Machine Learning and Data Mining in Pattern Recognition, volume 4571 of Lecture Notes in Computer Science, pages 61-75. Springer Berlin Heidelberg.

Laurikkala, J., Juhola, M., and Kentala, E. (2000). Informal identification of outliers in medical data.

Lazarevic, A. and Kumar, V. (2005). Feature bagging for outlier detection. pages 157-166, Chicago, IL.

Liu, F. T., Ting, K. M., and Zhou, Z.-H. (2012). Isolation-based anomaly detection. 6(1):3:1-39.

Micenková, B., van Beusekom, J., and Shafait, F. (2012). Stamp verification for automated document authentication. In 5th Int. Workshop on Computational Forensics.

Müller, E., Assent, I., Iglesias, P., Mülle, Y., and Böhm, K. (2012). Outlier ranking via subspace analysis in multiple views of the data. pages 529-538.

Müller, E., Schiffer, M., and Seidl, T. (2011). Statistical selection of relevant subspace projections for outlier ranking. pages 434-445.

Nguyen, H. V., Ang, H. H., and Gopalkrishnan, V. (2010). Mining outliers with ensemble of heterogeneous detectors on random subspaces. pages 368-383, Tsukuba, Japan. 
Nguyen, H. V. and Gopalkrishnan, V. (2010). Feature extraction for outlier detection in highdimensional spaces. In Liu, H., Motoda, H., Setiono, R., and Zhao, Z., editors, FSDM, volume 10 of JMLR Proceedings, pages 66-75. JMLR.org.

Orair, G. H., Teixeira, C., Wang, Y., Jr., W. M., and Parthasarathy, S. (2010). Distance-based outlier detection: Consolidation and renewed bearing. Proceedings of the VLDB Endowment, pages 3(2):1469-1480.

Papadimitriou, S., Kitagawa, H., Gibbons, P., and Faloutsos, C. (2003). Loci: Fast outlier detection using the local correlation integral. pages 315-326, Bangalore, India.

Prastawa, M., Bullitt, E., Ho, S., and Gerig, G. (2004). A brain tumor segmentation framework based on outlier detection. Medical Image Analysis, pages 275-283.

Quinlan, J. R. (1993). C4.5: Programs for Machine Learning. Morgan Kaufmann Publishers Inc., San Francisco, CA, USA.

Ramaswamy, S., Rastogi, R., and Shim, K. (2000). Efficient algorithms for mining outliers from large data sets. pages 427-438, Dallas, TX.

Schubert, E., Wojdanowski, R., Zimek, A., and Kriegel, H.-P. (2012). On evaluation of outlier rankings and outlier scores. pages 1047-1058, Anaheim, CA.

Schubert, E., Zimek, A., and Kriegel, H.-P. (2014a). Generalized outlier detection with flexible kernel density estimates. pages 542-550.

Schubert, E., Zimek, A., and Kriegel, H.-P. (2014b). Local outlier detection reconsidered: a generalized view on locality with applications to spatial, video, and network outlier detection. Data Mining and Knowledge Discovery, 28(1):190-237.

Song, X., Wu, M., Jermaine, C., and Ranka, S. (2007). Conditional anomaly detection. IEEE Transactions on Knowledge and Data Engineering, pages 19(5):631-645.

Sundar, C., Chitradevi, M., and Geetharamani, G. (2013). An overview of research challenges for classification of cardiotocogram data. Journal of Computer Science, 9(2):198-206.

Tang, J., Chen, Z., Fu, A. W.-C., and Cheung, D. W. (2002). Enhancing effectiveness of outlier detections for low density patterns. pages 535-548, Taipei, Taiwan.

Ting, K. M., Zhou, G.-T., Liu, F. T., and Tan, S. C. (2013). Mass estimation. Machine Learning, 90(1):127-160.

Wang, K. and Stolfo, S. (2004). Anomalous payload-based network intrusion detection. In Jonsson, E., Valdes, A., and Almgren, M., editors, Recent Advances in Intrusion Detection, volume 3224 of Lecture Notes in Computer Science, pages 203-222. Springer Berlin Heidelberg. 
Wang, X. and Davidson, I. (2009). Discovering contexts and contextual outliers using random walks in graphs. pages 1034-1039, Miami, FL.

Witten, I. H. and Frank, E. (2005). Data Mining: Practical machine learning tools and techniques. Morgan Kaufmann, 2nd edition.

Zhang, E. and Zhang, Y. (2009). Average precision. In Encyclopedia of Database Systems, pages 192-193.

Zhang, J. and Zulkernine, M. (2006). Anomaly based network intrusion detection with unsupervised outlier detection. In Communications, 2006. ICC '06. IEEE International Conference on, volume 5, pages 2388-2393.

Zhang, K., Hutter, M., and Jin, H. (2009). A new local distance-based outlier detection approach for scattered real-world data. pages 813-822, Bangkok, Thailand.

Zimek, A., Campello, R. J. G. B., and Sander, J. (2013a). Ensembles for unsupervised outlier detection: Challenges and research questions. SIGKDD Explor. Newsl., 15(1):11-22.

Zimek, A., Gaudet, M., Campello, R. J. G. B., and Sander, J. (2013b). Subsampling for efficient and effective unsupervised outlier detection ensembles. pages 428-436, Chicago, IL.

Zimek, A., Schubert, E., and Kriegel, H.-P. (2012). A survey on unsupervised outlier detection in high-dimensional numerical data. Statistical Analysis and Data Mining, pages 5(5):363387. 